\title{
Cu-Catalyzed Direct Diversification of 2-(2-Bromophenyl) Quinazolin-4(3H)-ones Through Orthogonal Reactivity Modulation
}

Satadru Chatterjee ${ }^{\# a}$, Ravuri Srinath ${ }^{\# b}$, Suvankar Bera ${ }^{a}$, Krishnendu Khamaru, Afifa Rahman and Biswadip Banerji ${ }^{\mathrm{a}, \mathrm{c} *}$

a Organic and Medicinal Chemistry Division, CSIR- Indian Institute of Chemical Biology (CSIR-IICB), Raja S.C. Mullick Road, Kolkata-70oo32 (India)

bNational Institute of Pharmaceutical Education and Research (NIPER-Kolkata), Chunilal Bhawan, Maniktala, Kolkata-700054 (India)

'Academy of Scientific and Innovative Research (AcSIR), CSIR-IICB; 4 Raja S. C. Mullick Road, Kolkata, Country. India-70oo32; Fax: (+) 9133 24735197, 9133 24723967; Tel: (+) 9133 24995709; E-mail: biswadip.banerji@gmail.com, biswadip@iicb.res.in

\section{Supporting Information}

\section{Table of Contents}

1 General Information S2

2 General procedure for the synthesis of compounds 3a-3i---------------------------S2-S7

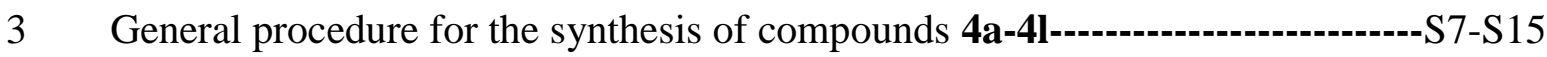

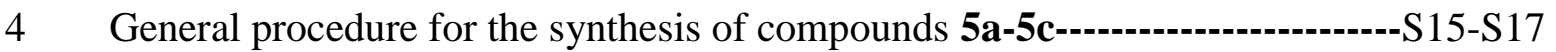

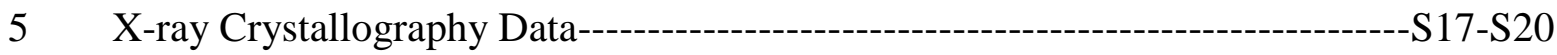

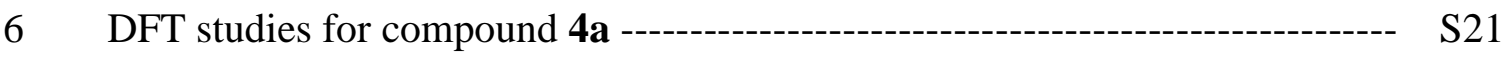

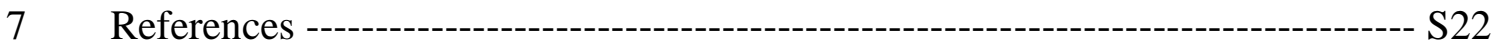

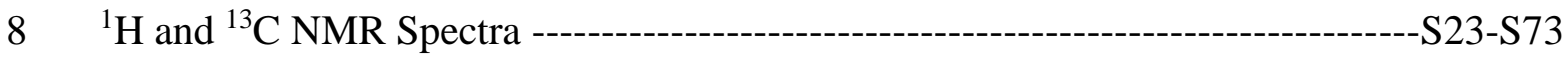




\section{General Information}

All the reagents and solvents used in this study were purchased from Sigma-Aldrich and Thermo Fischer Scientific, respectively. Melting points were recorded on a melting point apparatus in capillaries. All the ${ }^{1} \mathrm{H}$ and ${ }^{13} \mathrm{C}$ NMR spectra were recorded in a Bruker $600 \mathrm{MHz}$ spectrometer. ESI mass spectral analysis was done using LCQ-ORBITRAP-XL instrument. All UV and fluorescence data were collected using Jasco and Cary Eclipse spectrophotometers, respectively. Bruker Kappa Apex II X-ray crystallography machine was used to solve the crystal structure. Singlet (s), doublet (d), double doublet (dd), triplet (t), and multiplet (m) were designated as ${ }^{1} \mathrm{H}$ NMR multiplicity patterns. Silica gel (100-200 mesh) and (230-400 mesh) were used for column chromatographic separations.

\section{Structural determination by ROESY NMR:}

ROESY NMR spectrum of compound-3c was recorded in a Bruker Avance-600 MHz instrument with $5 \mathrm{~mm}$ TCI CYROPROBE in DMSO-d 6 using tetra-methylsilane (TMS) as internal standard in phase-sensitive mode. It was acquired with $2 \times 256$ free induction decays (FID) containing 8-16 scans with relaxation delays of $1.5 \mathrm{sec}$. and mixing time of 0.2 to 0.3 sec. Mnova was used to process the spectrum using Gaussian apodization in both the dimensions. ${ }^{1} \mathrm{H}^{1} \mathrm{H}$ ROESY cross peaks were assigned and integrated at $300 \mathrm{~ms}$, the respective volumes were converted to distance restraints.

\section{General procedure for the synthesis of compounds (3a-3i):}

To a solution of compound 1 ( $0.1 \mathrm{mmol}, 1.0$ equiv) and different amines $(0.15 \mathrm{mmol}, 1.2$ equiv) in dry DMSO $(1 \mathrm{~mL})$ was added $\mathrm{CuI}(20 \mathrm{~mol} \%)$ as catalyst, and $\mathrm{K}_{2} \mathrm{CO}_{3}$ (3 equiv) as base and stirred while heating at $120{ }^{\circ} \mathrm{C}$ in a sealed tube under $\mathrm{O}_{2}$ pressure. After completion of the reaction, monitored by TLC, the reaction mixture was diluted with ethyl acetate, filtered through Celite bed, and then washed with cold water $(4 \times 10 \mathrm{~mL})$ and brine $(2 \times 10$ $\mathrm{mL}$ ). The organic layer was then collected and dried over $\mathrm{Na}_{2} \mathrm{SO}_{4}$. After that, the organic extract was evaporated under reduced pressure to get crude solid. It was then subjected to column chromatography (silica gel 230-400 mesh size, ethyl acetate: pet ether) for further purification to get the desired compounds $(\mathbf{3 a}-\mathbf{3 i})$ in $69-90 \%$ yield. 


\section{Spectral Data:}

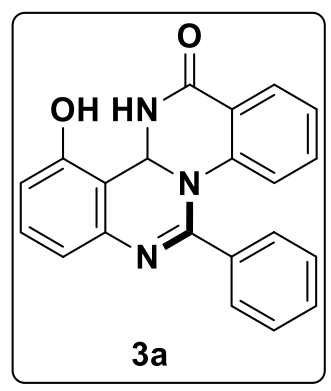

\section{1-hydroxy-6-phenyl-11bH-quinazolino[3,4-a]quinazolin-13(12H)-one (3a):}

The same general procedure was followed. Column chromatography $\left(\mathrm{SiO}_{2}\right.$, eluting with $20 \%$ ethyl acetate/pet ether) afforded the desired product as Pale yellow solid (29.92 mg, 88\% yield), mp $230-236{ }^{\circ} \mathrm{C}$.

${ }^{1} \mathrm{H}$ NMR (600 MHz, DMSO-d $\left.d_{6}\right) \delta 12.63(\mathrm{~s}, 1 \mathrm{H}), 12.44$ (s, 1H), 8.52 (d, $\left.J=6.0 \mathrm{~Hz}, 1 \mathrm{H}\right), 8.16$ $(\mathrm{d}, J=12.0 \mathrm{~Hz}, 1 \mathrm{H}), 7.95(\mathrm{dd}, J=6.0,6.0 \mathrm{~Hz}, 3 \mathrm{H}), 7.88(\mathrm{t}, J=6.0 \mathrm{~Hz}, 1 \mathrm{H}), 7.70(\mathrm{~d}, J=6.0$ $\mathrm{Hz}, 1 \mathrm{H}), 7.67-7.49(\mathrm{~m}, 5 \mathrm{H}), 7.29(\mathrm{t}, J=6.0 \mathrm{~Hz}, 1 \mathrm{H}) ;{ }^{13} \mathrm{C} \mathrm{NMR}(150 \mathrm{MHz}, \mathrm{DMSO}) \delta$ $165.43,162.49$, 153.71, 147.82, 138.54, 135.42, 135.39, 132.56, 132.33, 130.27, 129.30, 127.76, 127.56, 126.88, 126.51, 123.93, 121.87, 121.44, 121.30; HRMS (ESI): m/z calculated for $\mathrm{C}_{21} \mathrm{H}_{15} \mathrm{~N}_{3} \mathrm{O}_{2}[\mathrm{M}+\mathrm{H}]^{+}$342.1243; found 342.1244.<smiles>CCOc1ccc(C2C(=O)NC3c4ccccc4N2c2cccc(O)c23)cc1</smiles>

11-hydroxy-6-(4-methoxyphenyl)-11bH-quinazolino[3,4-a]quinazoline 13(12H)-one (3b)

The same general procedure was followed. Column chromatography $\left(\mathrm{SiO}_{2}\right.$, eluting with $20 \%$ ethyl acetate/pet ether) afforded the desired product as Pale yellow solid (28.12 mg, $76 \%$ yield), mp $250-241{ }^{\circ} \mathrm{C}$.

${ }^{1} \mathrm{H}$ NMR (600 MHz, DMSO-d $d_{6} \delta 12.61(\mathrm{~s}, 1 \mathrm{H}), 12.33$ (s, 1H), 8.49 (d, $\left.J=6.0 \mathrm{~Hz}, 1 \mathrm{H}\right), 8.16$ $(\mathrm{d}, J=6.0 \mathrm{~Hz}, 1 \mathrm{H}), 7.94-7.89(\mathrm{~m}, 4 \mathrm{H}), 7.73(\mathrm{~d}, J=6.0 \mathrm{~Hz}, 1 \mathrm{H}), 7.60-7.54(\mathrm{~m}, 2 \mathrm{H}), 7.26$ $(\mathrm{t}, J=6.0 \mathrm{~Hz}, 1 \mathrm{H}), 7.11-7.08(\mathrm{~m}, 2 \mathrm{H}), 3.83(\mathrm{~s}, 3 \mathrm{H}) ;{ }^{13} \mathrm{C} \mathrm{NMR}(150 \mathrm{MHz}, \mathrm{DMSO}) \delta 164.92$, $162.71,162.49,153.72,147.78,138.72,135.55,132.31,130.23,129.69,127.59,127.45$, 
126.93, 126.50, 123.70, 121.84, 121.38, 121.09, 114.52, 55.99; HRMS (ESI): m/z calculated for $\mathrm{C}_{22} \mathrm{H}_{16} \mathrm{~N}_{3} \mathrm{O}_{3}[\mathrm{M}+\mathrm{H}]^{+}$372.1348; found 372.1349.

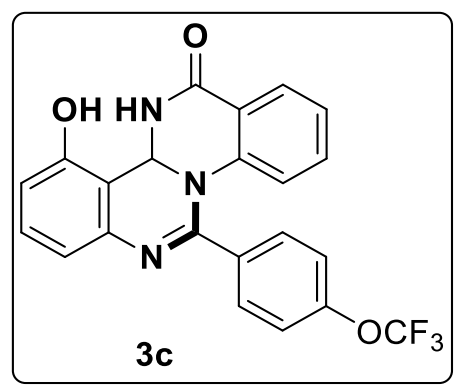

\section{1-hydroxy-6-(4-(trifluoromethoxy)phenyl)-11bH-quinazolino[3,4-a]quinazolin- 13(12H)-one (3c)}

The same general procedure was followed. Column chromatography $\left(\mathrm{SiO}_{2}\right.$, eluting with $17 \%$ ethyl acetate/pet ether) afforded the desired product as Pale yellow solid (22 $\mathrm{mg}, 80 \%$ yield), $\operatorname{mp} 230-231^{\circ} \mathrm{C}$.

${ }^{1} \mathrm{H}$ NMR (600 MHz, DMSO-d6) $\delta 12.62(\mathrm{~s}, 1 \mathrm{H}), 12.28(\mathrm{~s}, 1 \mathrm{H}), 8.41$ (d, $\left.J=6.0 \mathrm{~Hz}, 1 \mathrm{H}\right), 8.16$ - $8.06(\mathrm{~m}, 3 \mathrm{H}), 7.93-7.84(\mathrm{~m}, 2 \mathrm{H}), 7.69(\mathrm{~d}, J=12 \mathrm{~Hz}, 1 \mathrm{H}), 7.61-7.53(\mathrm{~m}, 4 \mathrm{H}), 7.30$ (t, $J=$ $12.0 \mathrm{~Hz}, 1 \mathrm{H}) ;{ }^{13} \mathrm{C}$ NMR (150 MHz, DMSO) $\delta 164.26,162.48,153.59,151.16,147.90$, $138.11,135.36,134.54,131.26\left(\mathrm{q},{ }^{1} J_{\mathrm{C}-\mathrm{F}}=285.0 \mathrm{~Hz}\right), 130.15,127.50,126.99,126.44,124.25$, 122.23, 122.01, 121.52, 121.27, 120.57 (q, $\left.{ }^{1} J_{\mathrm{C}-\mathrm{F}}=279.0 \mathrm{~Hz}\right) ;$ HRMS (ESI-TOF): $\mathrm{m} / \mathrm{z}$ $[\mathrm{M}+\mathrm{H}]^{+}$calculated for $\mathrm{C}_{22} \mathrm{H}_{14} \mathrm{~F}_{3} \mathrm{~N}_{3} \mathrm{O}_{3} 426.1065$; found 426.1067 .

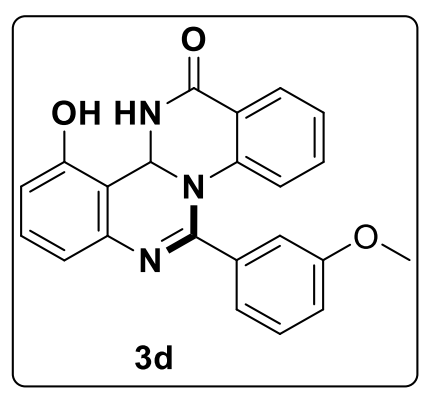

\section{1-hydroxy-6-(3-methoxyphenyl)-11bH-quinazolino[3,4-a]quinazolin-13(12H)-one (3d)}

The same general procedure was followed. Column chromatography $\left(\mathrm{SiO}_{2}\right.$, eluting with $18 \%$ ethyl acetate/pet ether) afforded the desired product as Pale yellow solid (30.01 mg, 78\% yield), mp $210-212^{\circ} \mathrm{C}$.

${ }^{1} \mathrm{H}$ NMR (400 MHz, DMSO-d $\left.)_{6}\right) \delta 12.58$ (s, 1H), 11.59 (s, 1H), 8.43 (d, $\left.J=8.0 \mathrm{~Hz}, 1 \mathrm{H}\right), 8.13$ $(\mathrm{d}, J=8.0 \mathrm{~Hz}, 1 \mathrm{H}), 7.80-7.75(\mathrm{~m}, 3 \mathrm{H}), 7.54-7.46(\mathrm{~m}, 4 \mathrm{H}), 7.24(\mathrm{t}, J=8.0 \mathrm{~Hz}, 1 \mathrm{H}), 7.10$ 
$(\mathrm{d}, J=8.0 \mathrm{~Hz}, 1 \mathrm{H}), 7.04(\mathrm{t}, J=8.0 \mathrm{~Hz}, 1 \mathrm{H}), 3.49$ (s, 3H); ${ }^{13} \mathrm{C}$ NMR (100 MHz, DMSO) $\delta$ 167.94 , 164.30, 157.10, 146.23, 137.76, 135.31, 134.35, 133.58, 131.93, 131.28, 130.32, 127.63, 127.32, 126.46, 124.06, 121.45, 121.36, 118.75, 112.73, 112.48, 56.51; HRMS (ESITOF) : m/z [M+H] $]^{+}$calculated for $\mathrm{C}_{22} \mathrm{H}_{17} \mathrm{~N}_{3} \mathrm{O}_{3}$ 372.1348; found 372.1349.

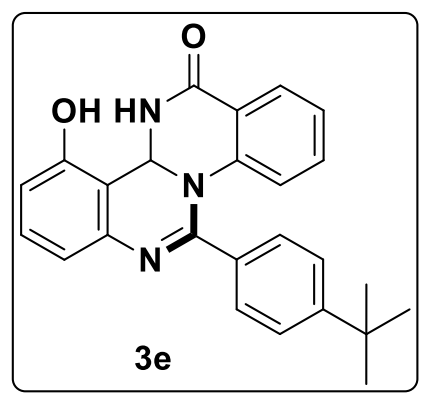

\section{6-(4-(tert-butyl)phenyl)-11-hydroxy-11bH-quinazolino[3,4-a]quinazolin-13(12H)-one} (3e)

The same general procedure was followed. Column chromatography $\left(\mathrm{SiO}_{2}\right.$, eluting with $16 \%$ ethyl acetate/pet ether) afforded the desired product as Pale yellow solid (27.30 mg, $70 \%$ yield), mp $215-217^{\circ} \mathrm{C}$.

${ }^{1} \mathrm{H}$ NMR (600 MHz, DMSO-d $) \delta 12.62(\mathrm{~s}, 1 \mathrm{H}), 12.32(\mathrm{~s}, 1 \mathrm{H}), \delta 8.50(\mathrm{~d}, J=6.0 \mathrm{~Hz}, 1 \mathrm{H})$, $8.16(\mathrm{~d}, J=6.0 \mathrm{~Hz}, 1 \mathrm{H}), 7.93-7.88(\mathrm{~m}, 4 \mathrm{H}), 7.75(\mathrm{~d}, J=6.0 \mathrm{~Hz}, 1 \mathrm{H}), 7.60-7.54(\mathrm{~m}, 4 \mathrm{H})$, $7.28(\mathrm{t}, J=6.0 \mathrm{~Hz}, 1 \mathrm{H}), 1.30(\mathrm{~s}, 9 \mathrm{H}) ;{ }^{13} \mathrm{C}$ NMR (150 MHz, DMSO) $\delta 165.25,162.45,155.45$, $153.66,147.82$, 138.52, 135.39, 132.62, 132.25, 130.27, 128.17, 127.62, 127.56, 126.96, 126.48, 126.03, 123.83, 121.90, 121.42, 121.38, 35.22, 31.38; HRMS (ESI-TOF) : m/z $[\mathrm{M}+\mathrm{H}]^{+}$calculated for $\mathrm{C}_{25} \mathrm{H}_{24} \mathrm{~N}_{3} \mathrm{O}_{2}$ 398.1868; found 398.1869.

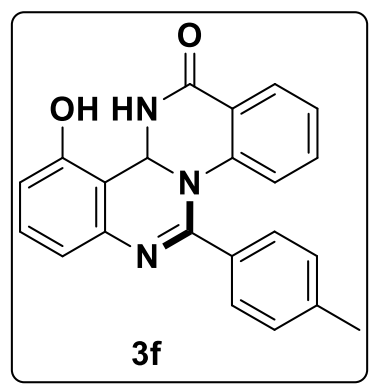

11-hydroxy-6-(p-tolyl)-11bH-quinazolino[3,4-a]quinazolin-13(12H)-one (3f) 
The same general procedure was followed. Column chromatography $\left(\mathrm{SiO}_{2}\right.$, eluting with $18 \%$ ethyl acetate/pet ether) afforded the desired product as Pale yellow solid (26.65 mg, 75\% yield), mp $240-242{ }^{\circ} \mathrm{C}$.

${ }^{1} \mathrm{H}$ NMR (600 MHz, DMSO-d6) $\delta 12.62(\mathrm{~s}, 1 \mathrm{H}), 12.38(\mathrm{~s}, 1 \mathrm{H}), 8.52(\mathrm{~d}, J=12.0 \mathrm{~Hz}, 1 \mathrm{H})$, $8.17(\mathrm{~d}, J=6.0 \mathrm{~Hz}, 1 \mathrm{H}), 7.93-7.86(\mathrm{~m}, 4 \mathrm{H}), 7.73(\mathrm{~d}, J=6.0 \mathrm{~Hz}, 1 \mathrm{H}), 7.60-7.55(\mathrm{~m}, 2 \mathrm{H})$, $7.36(\mathrm{~d}, J=6.0 \mathrm{~Hz}, 2 \mathrm{H}), 7.28(\mathrm{t}, J=6.0 \mathrm{~Hz}, 1 \mathrm{H}), 2.38(\mathrm{~s}, 3 \mathrm{H}) ;{ }^{13} \mathrm{C}$ NMR (151 MHz, DMSO) $\delta 165.25,142.71,138.57,135.59,135.46,132.49,132.31,132.17,130.25,129.80,127.76$, 127.56, 126.84, 126.51, 123.82, 121.82, 121.40, 121.34, 121.19, 21.51; HRMS (ESI-TOF) : $\mathrm{m} / \mathrm{z}[\mathrm{M}+\mathrm{H}]^{+}$calculated for $\mathrm{C}_{22} \mathrm{H}_{17} \mathrm{~N}_{3} \mathrm{O}_{2} 356.1399$; found 356.1403.

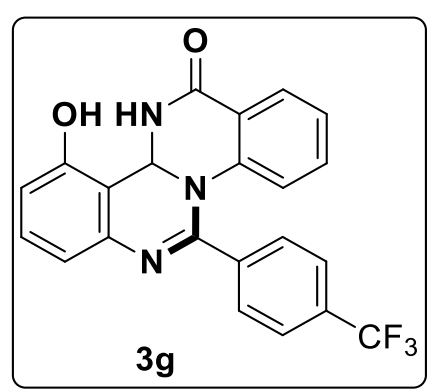

\section{1-hydroxy-6-(4-(trifluoromethyl)phenyl)-11bH-quinazolino[3,4 a]quinazolin-13(12H)- one (3g)}

The same general procedure was followed. Column chromatography $\left(\mathrm{SiO}_{2}\right.$, eluting with $16 \%$ ethyl acetate/pet ether) afforded the desired product as Pale yellow solid (34.77 mg, 85\% yield), mp 233-234 ${ }^{\circ} \mathrm{C}$.

${ }^{1} \mathrm{H}$ NMR (600 MHz, DMSO-d $\left.)\right) \delta 12.63(\mathrm{~s}, 1 \mathrm{H}), 12.29(\mathrm{~s}, 1 \mathrm{H}), 8.41(\mathrm{~d}, J=12.0 \mathrm{~Hz}, 1 \mathrm{H})$, $8.17-8.12(\mathrm{~m}, 4 \mathrm{H}), 7.93(\mathrm{~d}, J=6.0 \mathrm{~Hz}, 3 \mathrm{H}), 7.87(\mathrm{t}, J=6.0 \mathrm{~Hz}, 1 \mathrm{H}), 7.71(\mathrm{~d}, J=12.0 \mathrm{~Hz}$, $1 \mathrm{H}), 7.61(\mathrm{t}, J=12.0 \mathrm{~Hz}, 1 \mathrm{H}), 7.54(\mathrm{t}, J=12.0 \mathrm{~Hz}, 1 \mathrm{H}), 7.32(\mathrm{t}, J=12.0 \mathrm{~Hz}, 1 \mathrm{H}) ;{ }^{13} \mathrm{C} \mathrm{NMR}$ (150 MHz, DMSO) $\delta 164.28,162.52,153.58,147.96,139.16,137.88,135.44,132.28$, $132.20,132.07,130.38,128.74,127.51,127.04,126.44,126.26,125.23,124.48,123.42$, 122.42, 121.43, 40.38; HRMS (ESI): $\mathrm{m} / \mathrm{z}$ calculated for $\mathrm{C}_{22} \mathrm{H}_{14} \mathrm{~F}_{3} \mathrm{~N}_{3} \mathrm{O}_{2}[\mathrm{M}+\mathrm{H}]^{+} 410.1116$; found 410.1119 . 


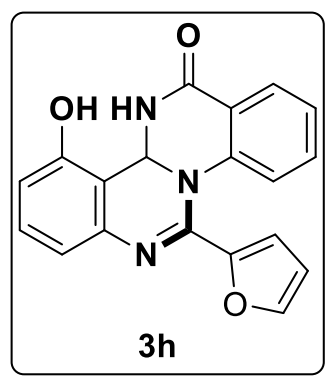

\section{6-(furan-2-yl)-11-hydroxy-11bH-quinazolino[3,4-a]quinazolin-13(12H)-one (3h)}

The same general procedure was followed. Column chromatography $\left(\mathrm{SiO}_{2}\right.$, eluting with $20 \%$ ethyl acetate/pet ether) afforded the desired product as Pale yellow solid (25.16 mg, 76\% yield), mp 197-199 ${ }^{\circ} \mathrm{C}$.

${ }^{1} \mathrm{H}$ NMR (600 MHz, DMSO-d6) $\delta 13.17(\mathrm{~s}, 1 \mathrm{H}), 12.62(\mathrm{~s}, 1 \mathrm{H}), \delta 8.59(\mathrm{~d}, J=6.0 \mathrm{~Hz}, 1 \mathrm{H})$, $8.18(\mathrm{~d}, J=6.0 \mathrm{~Hz}, 1 \mathrm{H}), 8.08(\mathrm{~d}, J=6.0 \mathrm{~Hz}, 1 \mathrm{H}), 8.03(\mathrm{~d}, J=6.0 \mathrm{~Hz}, 1 \mathrm{H}), 7.96-7.91(\mathrm{~m}$, $2 \mathrm{H}), 7.61-7.58(\mathrm{~m}, 2 \mathrm{H}), 7.29-7.26(\mathrm{~m}, 2 \mathrm{H}), 6.76-6.73(\mathrm{~m}, 1 \mathrm{H}) ;{ }^{13} \mathrm{C} \mathrm{NMR}(150 \mathrm{MHz}$, DMSO) $\delta 162.45,156.16,153.29,152.65,148.31,147.53,146.50,138.48,135.69,132.58$, 130.06, 127.70, 127.12, 126.44, 123.78, 121.40, 119.75, 115.95, 113.29; HRMS (ESI-TOF) : $\mathrm{m} / \mathrm{z}[\mathrm{M}+\mathrm{H}]^{+}$calculated for $\mathrm{C}_{19} \mathrm{H}_{14} \mathrm{~N}_{3} \mathrm{O}_{3} 332.1035$; found 332.1034 .

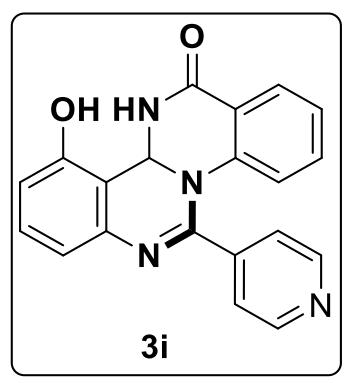

\section{1-hydroxy-6-(pyridin-4-yl)-11bH-quinazolino[3,4-a]quinazolin-13(12H)-one (3i)}

The same general procedure was followed. Column chromatography $\left(\mathrm{SiO}_{2}\right.$, eluting with $20 \%$ ethyl acetate/pet ether) afforded the desired product as Pale yellow solid (19.01 mg, 55\% yield), mp $270-271{ }^{\circ} \mathrm{C}$.

${ }^{1} \mathrm{H}$ NMR (600 MHz, DMSO-d $\left.d_{6}\right) \delta 12.64(\mathrm{~s}, 1 \mathrm{H}), 12.36$ (s, 1H), 8.80 (d, $\left.J=6.0 \mathrm{~Hz}, 2 \mathrm{H}\right), 8.41$ $(\mathrm{d}, J=6.0 \mathrm{~Hz}, 1 \mathrm{H}), 8.16(\mathrm{~d}, J=6.0 \mathrm{~Hz}, 1 \mathrm{H}), 7.94(\mathrm{~d}, J=6.0 \mathrm{~Hz}, 1 \mathrm{H}), 7.90-7.80(\mathrm{~m}, 3 \mathrm{H})$, $7.68(\mathrm{~d}, J=6.0 \mathrm{~Hz}, 1 \mathrm{H}), 7.63-7.60(\mathrm{~m}, 1 \mathrm{H}), 7.55(\mathrm{t}, J=6.0 \mathrm{~Hz}, 1 \mathrm{H}), 7.33$ (t, $J=6.0 \mathrm{~Hz}$, $1 \mathrm{H}) ;{ }^{13} \mathrm{C}$ NMR (150 MHz, DMSO) $\delta 164.02,162.61,159.03,151.08,148.01,142.48,137.74$, 
$136.46,135.35,132.23,130.39,129.69,127.49,127.01,126.46,124.64,122.40,121.72$; HRMS (ESI-TOF) : m/z [M+H] $]^{+}$calculated for $\mathrm{C}_{20} \mathrm{H}_{14} \mathrm{~N}_{4} \mathrm{O}_{2} 343.1195$; found 343.1196 .

\section{General procedure for the synthesis of compounds (4a-4l):}

To a solution of compound $\mathbf{1}(0.1 \mathrm{mmol}, 1.0$ equiv) and different amines $(0.15 \mathrm{mmol}, 1.2$ equiv) in dry DMSO $(1 \mathrm{~mL})$ was added $\mathrm{CuI}(20 \mathrm{~mol} \%)$ as catalyst, and $\mathrm{K}_{2} \mathrm{CO}_{3}$ (3 equiv) as base and stirred while heating at $120^{\circ} \mathrm{C}$ in a sealed tube. After completion of the reaction, monitored by TLC, the reaction mixture was diluted with ethyl acetate, filtered through Celite bed, and then washed with cold water $(4 \times 10 \mathrm{~mL})$ and brine $(2 \times 10 \mathrm{~mL})$. The organic layer was then collected and dried over $\mathrm{Na}_{2} \mathrm{SO}_{4}$. After that, the organic extract was evaporated under reduced pressure to get crude solid. It was then subjected to column chromatography (silica gel 230-400 mesh size, ethyl acetate: pet ether) for further purification to get the desired compounds $(\mathbf{4 a - 4 l )}$ in $69-90 \%$ yield

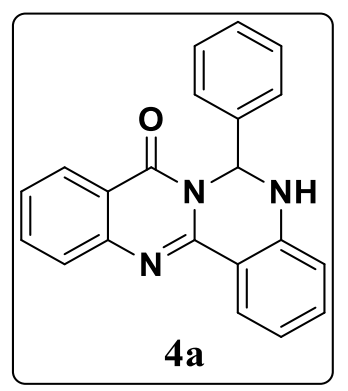

\section{6-phenyl-5H-quinazolino[4,3-b]quinazolin-8(6H)-one (4a)}

The same general procedure was followed. Column chromatography $\left(\mathrm{SiO}_{2}\right.$, eluting with $9 \%$ ethyl acetate/pet ether) afforded the desired product as Pale yellow solid (28.93 mg, 89\% yield), mp $224-225^{\circ} \mathrm{C}$.

Pale yellow solid, m.p., ${ }^{1} \mathrm{H}$ NMR (600 MHz, DMSO-d6) $\delta 8.19(\mathrm{~d}, J=6.0 \mathrm{~Hz}, 1 \mathrm{H}), 8.12-$ $8.09(\mathrm{~m}, 1 \mathrm{H}), 8.00(\mathrm{~d}, J=6.0 \mathrm{~Hz}, 1 \mathrm{H}), 7.88-7.83(\mathrm{~m}, 1 \mathrm{H}), 7.74(\mathrm{~d}, J=6.0 \mathrm{~Hz}, 1 \mathrm{H}), 7.51(\mathrm{t}$, $J=6.0 \mathrm{~Hz}, 1 \mathrm{H}), 7.35-7.29(\mathrm{~m}, 1 \mathrm{H}), 7.27-7.20(\mathrm{~m}, 4 \mathrm{H}), 7.16(\mathrm{~d}, J=6.0 \mathrm{~Hz}, 2 \mathrm{H}), 6.91(\mathrm{~d}, J$ $=6.0 \mathrm{~Hz}, 1 \mathrm{H}), 6.81(\mathrm{t}, J=6.0 \mathrm{~Hz}, 1 \mathrm{H}) ;{ }^{13} \mathrm{C} \mathrm{NMR}(150 \mathrm{MHz}, \mathrm{DMSO}) \delta 160.12,148.16$, $147.71,145.58,139.90,135.46,134.15,129.01,128.61,127.71,127.33,127.11,126.70$, 126.11, 120.35, 119.22, 116.33, 116.01, 62.90. HRMS (ESI-TOF): m/z [M+H] ${ }^{+}$calculated for $\mathrm{C}_{21} \mathrm{H}_{16} \mathrm{~N}_{3} \mathrm{O}$ 326.1293; found 326.1295. 


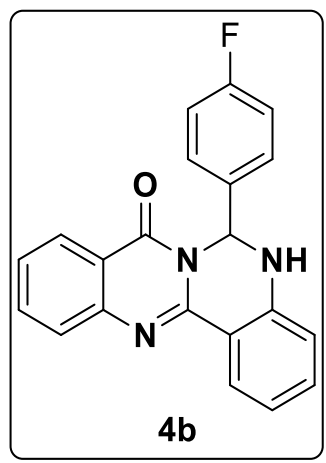

\section{6-(4-fluorophenyl)-5H-quinazolino[4,3-b]quinazolin-8(6H)-one (4b)}

The same general procedure was followed. Column chromatography $\left(\mathrm{SiO}_{2}\right.$, eluting with $10 \%$ ethyl acetate/pet ether) afforded the desired product as Pale yellow solid (24.70 $\mathrm{mg}, 72 \%$ yield), mp $210-212^{\circ} \mathrm{C}$.

${ }^{1} \mathrm{H}$ NMR $\left(600 \mathrm{MHz}, \mathrm{DMSO}-d_{6}\right) \delta=8.14(\mathrm{~d}, J=6.0 \mathrm{~Hz}, 2 \mathrm{H}), 7.97(\mathrm{~s}, 1 \mathrm{H}), 7.85$ (t, $J=6.0 \mathrm{~Hz}$, 1H), $7.73(\mathrm{~d}, J=6.0 \mathrm{~Hz}, 1 \mathrm{H}), 7.51(\mathrm{t}, J=6.0 \mathrm{~Hz}, 1 \mathrm{H}), 7.35-7.32(\mathrm{~m}, 1 \mathrm{H}), 7.21(\mathrm{~d}, J=6.0 \mathrm{~Hz}$, $3 \mathrm{H}), 7.09$ (t, $J=6.0 \mathrm{~Hz}, 2 \mathrm{H}), 6.91(\mathrm{~d}, J=6.0 \mathrm{~Hz}, 1 \mathrm{H}), 6.82$ (t, $J=6.0 \mathrm{~Hz}, 1 \mathrm{H}) .{ }^{13} \mathrm{C} \mathrm{NMR}(150$ MHz, DMSO) $\delta=160.10,148.14,147.53,145.37,136.12,135.52,134.24,128.33,128.27$, $127.71,127.36,127.11,126.76,120.31,119.38,116.39,115.96,115.82,62.43$. HRMS (ESITOF): $\mathrm{m} / \mathrm{z}[\mathrm{M}+\mathrm{H}]^{+}$calculated for $\mathrm{C}_{21} \mathrm{H}_{15} \mathrm{FN}_{3} \mathrm{O} 344.1199$; found 344.1200.

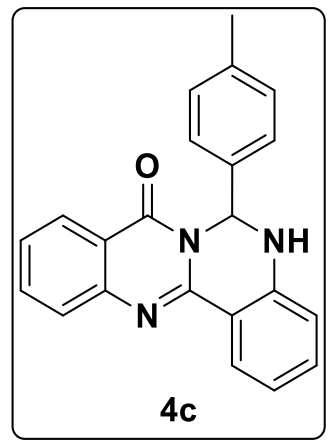

\section{6-(p-tolyl)-5H-quinazolino[4,3-b]quinazolin-8(6H)-one $(4 c)$}

The same general procedure was followed. Column chromatography $\left(\mathrm{SiO}_{2}\right.$, eluting with $8 \%$ ethyl acetate/pet ether) afforded the desired product as Pale yellow solid (25.01 $\mathrm{mg}, 75 \%$ yield), mp $240-242{ }^{\circ} \mathrm{C}$.

${ }^{1} \mathrm{H}$ NMR $\left(600 \mathrm{MHz}, \mathrm{DMSO}-d_{6}\right) \delta=8.20-8.16(\mathrm{~m}, 2 \mathrm{H}), 8.10(\mathrm{~d}, J=6.0 \mathrm{~Hz}, 1 \mathrm{H}), 7.95(\mathrm{~d}$, $J=6.0 \mathrm{~Hz}, 1 \mathrm{H}), 7.88-7.81(\mathrm{~m}, 2 \mathrm{H}), 7.73(\mathrm{~d}, J=6.0,6.0 \mathrm{~Hz}, 2 \mathrm{H}), 7.60-7.48(\mathrm{~m}, 3 \mathrm{H}), 7.31$ (d, $J=6.0 \mathrm{~Hz}, 1 \mathrm{H}), 7.20$ (d, J=6.0 Hz, 1H), 6.90 (d, J=6.0 Hz, 1H), $6.82-6.79$ (m, 1H), 2.16 (s, 
$3 \mathrm{H}) .{ }^{13} \mathrm{C}$ NMR (150 MHz, DMSO) $\delta=160.08,148.16,147.75,145.65,137.94,136.92$, 135.43 , 134.10, 129.52, 129.04, 128.19, 127.69, 127.31, 127.10, 126.67, 126.05, 120.36, 119.15, 116.32, 116.06, 62.77, 20.94. HRMS (ESI-TOF): $\mathrm{m} / \mathrm{z}[\mathrm{M}+\mathrm{H}]^{+}$calculated for $\mathrm{C}_{22} \mathrm{H}_{18} \mathrm{~N}_{3} \mathrm{O} 340.4060$; found 340.4061 .<smiles>CCCc1ccc(C2Nc3ccccc3-c3nc4ccccc4c(=O)n32)cc1</smiles>

\section{6-(4-(trifluoromethyl)phenyl)-5H-quinazolino[4,3-b]quinazolin-8(6H)-one (4d)}

The same general procedure was followed. Column chromatography $\left(\mathrm{SiO}_{2}\right.$, eluting with $7 \%$ ethyl acetate/pet ether) afforded the desired product as Pale yellow solid (27.12 $\mathrm{mg}, 69 \%$ yield), mp $230-232{ }^{\circ} \mathrm{C}$.

${ }^{1} \mathrm{H}$ NMR (600 MHz, DMSO- $\left.d_{6}\right) \delta=8.15(\mathrm{~d}, J=6.0 \mathrm{~Hz}, 2 \mathrm{H}), 7.95-7.81(\mathrm{~m}, 2 \mathrm{H}), 7.73(\mathrm{~d}$, $J=6.0 \mathrm{~Hz}, 1 \mathrm{H}), 7.50(\mathrm{~m}, 1 \mathrm{H}), 7.33(\mathrm{~m}, 1 \mathrm{H}), 7.15(\mathrm{~s}, 1 \mathrm{H}), 6.91(\mathrm{~d}, J=6.0 \mathrm{~Hz}, 1 \mathrm{H}), 6.82(\mathrm{~s}, 2 \mathrm{H})$, $6.73(\mathrm{~d}, J=6.0 \mathrm{~Hz}, 1 \mathrm{H}), 6.47(\mathrm{~d}, J=6.0 \mathrm{~Hz}, 1 \mathrm{H}), 5.93(\mathrm{~d}, J=6.0 \mathrm{~Hz}, 2 \mathrm{H}) ;{ }^{13} \mathrm{C} \mathrm{NMR}(150 \mathrm{MHz}$, DMSO- $\left.d_{6}\right) \delta=160.09,148.15,147.75,145.62,137.98,135.46,134.12,129.52,127.68$, $127.31,127.10,126.69,126.04,119.19,116.32,116.05,62.77,20.93$; HRMS (ESI-TOF) : $\mathrm{m} / \mathrm{z}[\mathrm{M}+\mathrm{H}]^{+}$calculated for $\mathrm{C}_{22} \mathrm{H}_{15} \mathrm{~F}_{3} \mathrm{~N}_{3} \mathrm{O} 394.1167$; found 394.1169 .

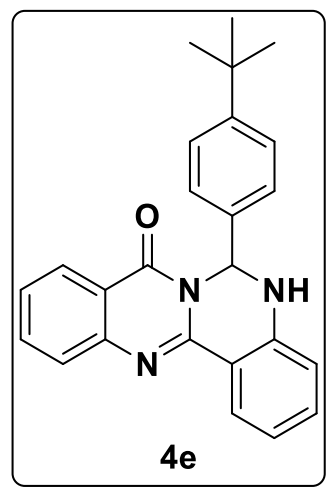

6-(4-(tert-butyl)phenyl)-5H-quinazolino[4,3-b]quinazolin-8(6H)-one (4e) 
The same general procedure was followed. Column chromatography $\left(\mathrm{SiO}_{2}\right.$, eluting with $12 \%$ ethyl acetate/pet ether) afforded the desired product as Pale yellow solid $(30.11 \mathrm{mg}, 79 \%$ yield), mp $230-232{ }^{\circ} \mathrm{C}$.

${ }^{1} \mathrm{H}$ NMR $\left(600 \mathrm{MHz}, \mathrm{DMSO}-d_{6}\right) \delta=8.17(\mathrm{~d}, J=6.0 \mathrm{~Hz}, 1.5,1 \mathrm{H}), 8.13-8.10(\mathrm{~m}, 1 \mathrm{H}), 7.96(\mathrm{~d}$, $J=6.0 \mathrm{~Hz}, 1 \mathrm{H}), 7.85$ (t, $J=6.0 \mathrm{~Hz}, 1 \mathrm{H}), 7.73$ (d, $J=6.0 \mathrm{~Hz}, 1 \mathrm{H}), 7.50$ (t, $J=6.0 \mathrm{~Hz}, 1 \mathrm{H}), 7.34-$ $7.30(\mathrm{~m}, 1 \mathrm{H}), 7.28-7.25(\mathrm{~m}, 2 \mathrm{H}), 7.19$ (d, J=6.0 Hz, 1H), 7.08 (d, J=6.0 Hz, 2H), 6.90 (d, $J=6.0 \mathrm{~Hz}, 1 \mathrm{H}), 6.81(\mathrm{t}, J=6.0 \mathrm{~Hz}, 1 \mathrm{H}), 1.15(\mathrm{~d}, J=6.0 \mathrm{~Hz}, 9 \mathrm{H}) .{ }^{13} \mathrm{C}$ NMR $(150 \mathrm{MHz}, \mathrm{DMSO})$ $\delta=160.08,151.08,148.16,147.73,145.64,137.04,135.44,134.17,127.68,127.37,127.08$, 126.66, 125.84, 120.37, 119.12, 116.29, 115.86, 62.80, 34.62, 31.34. HRMS (ESI-TOF): $\mathrm{m} / \mathrm{z}[\mathrm{M}+\mathrm{H}]^{+}$calculated for $\mathrm{C}_{25} \mathrm{H}_{24} \mathrm{~N}_{3} \mathrm{O} 382.1919$; found 382.1920 .

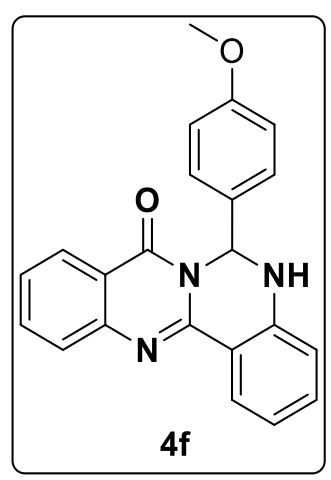

\section{6-(4-methoxyphenyl)-5H-quinazolino[4,3-b]quinazolin-8(6H)-one (4f)}

The same general procedure was followed. Column chromatography $\left(\mathrm{SiO}_{2}\right.$, eluting with $12 \%$ ethyl acetate/pet ether) afforded the desired product as Pale yellow solid (31.60 mg, 89\% yield), mp $210-212{ }^{\circ} \mathrm{C}$.

${ }^{1} \mathrm{H}$ NMR $\left(600 \mathrm{MHz}, \mathrm{DMSO}-d_{6}\right) \delta=12.21(\mathrm{~s}, 1 \mathrm{H}), 9.24(\mathrm{t}, J=6.0 \mathrm{~Hz}, 1 \mathrm{H}), 8.13(\mathrm{~d}, J=6.0 \mathrm{~Hz}$, 1H), $7.89-7.77(\mathrm{~m}, 2 \mathrm{H}), 7.60(\mathrm{~d}, J=6.0 \mathrm{~Hz}, 1 \mathrm{H}), 7.48(\mathrm{~m}, 1 \mathrm{H}), 7.26(\mathrm{t}, J=6.0 \mathrm{~Hz}, 2 \mathrm{H}), 7.00$ $(\mathrm{d}, J=6.0 \mathrm{~Hz}, 2 \mathrm{H}), 6.83(\mathrm{~d}, J=6.0 \mathrm{~Hz}, 1 \mathrm{H}), 6.77-6.61(\mathrm{~m}, 2 \mathrm{H}), 3.71(\mathrm{~s}, 3 \mathrm{H}) ;{ }^{13} \mathrm{C} \mathrm{NMR}(150$ MHz, DMSO-d6) $\delta=162.44,159.91,154.01,141.61,135.00$, $132.81,130.04$, 129.68 , $127.07,126.84,126.22$, 121.00 , $119.64,115.29,113.35,112.55$, 112.22 , 55.40 , 46.67; HRMS (ESI-TOF) : $\mathrm{m} / \mathrm{z}[\mathrm{M}+\mathrm{H}]^{+}$calculated for $\mathrm{C}_{22} \mathrm{H}_{18} \mathrm{~N}_{3} \mathrm{O}_{2} 356.1399$; found 356.1401.

\section{2-(2-((4-methoxybenzyl)amino)phenyl)quinazolin-4(3H)-one (4f'):}




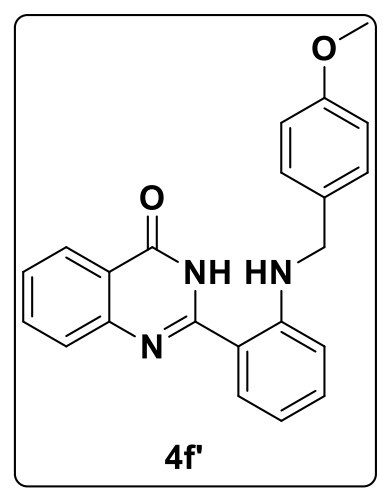

The same general procedure was followed. Column chromatography $\left(\mathrm{SiO}_{2}\right.$, eluting with $7 \%$ ethyl acetate/pet ether) afforded the desired product as smooth yellow powder (18 mg, 40\% yield), mp $150-153^{\circ} \mathrm{C}$.

${ }^{1} \mathrm{H}$ NMR $\left(600 \mathrm{MHz}, \mathrm{DMSO}-d_{6}\right) \delta 12.21(\mathrm{~s}, 1 \mathrm{H}), 9.24(\mathrm{t}, J=6.0 \mathrm{~Hz}, 1 \mathrm{H}), 8.18-8.12(\mathrm{~m}, 1 \mathrm{H})$, 7.83-7.79 (m, 2H), $7.60(\mathrm{~d}, J=6.0 \mathrm{~Hz}, 1 \mathrm{H}), 7.54-7.47(\mathrm{~m}, 1 \mathrm{H}), 7.27(\mathrm{t}, J=6.0 \mathrm{~Hz}, 2 \mathrm{H}), 6.99$ $(\mathrm{s}, 1 \mathrm{H}), 6.83(\mathrm{~s}, 1 \mathrm{H}), 6.72-6.61(\mathrm{~m}, 2 \mathrm{H}), 4.47(\mathrm{~s}, 2 \mathrm{H}), 3.71(\mathrm{~s}, 3 \mathrm{H}) ;{ }^{13} \mathrm{C}$ NMR $(150 \mathrm{MHz}$, DMSO) $\delta 162.44,159.91,154.01,148.11,141.61,135.00,132.81,134.12,130.04,129.68$, $127.07,126.84,126.22,121.00,119.64,115.29,113.35,112.55,112.22,55.40,46.67$; HRMS (ESI-TOF) : m/z [M + H] $]^{+}$calculated for $\mathrm{C}_{22} \mathrm{H}_{20} \mathrm{~N}_{3} \mathrm{O}_{2}$ 358.1555; found 358.1558.

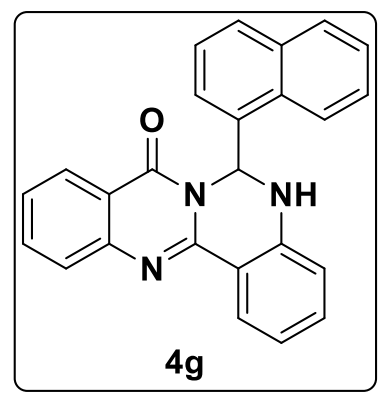

\section{6-(naphthalen-1-yl)-5H-quinazolino[4,3-b]quinazolin-8(6H)-one (4g)}

The same general procedure was followed. Column chromatography $\left(\mathrm{SiO}_{2}\right.$, eluting with $10 \%$ ethyl acetate/pet ether) afforded the desired product as Pale yellow solid (33.76 mg, 90\% yield), mp $230-232{ }^{\circ} \mathrm{C}$.

${ }^{1} \mathrm{H}$ NMR (400 MHz, DMSO- $\left.d_{6}\right) \delta 8.53(\mathrm{~d}, J=8.0 \mathrm{~Hz}, 1 \mathrm{H}), 8.23(\mathrm{~d}, J=8.0 \mathrm{~Hz}, 1 \mathrm{H}), 8.03(\mathrm{~d}$, $J=8.0 \mathrm{~Hz}, 1 \mathrm{H}), 7.96-7.92(\mathrm{~m}, 2 \mathrm{H}), 7.86-7.76(\mathrm{~m}, 3 \mathrm{H}), 7.71-7.67(\mathrm{~m}, 2 \mathrm{H}), 7.58(\mathrm{t}, J=8.0 \mathrm{~Hz}$, $1 \mathrm{H}), 7.44(\mathrm{t}, J=8.0 \mathrm{~Hz}, 1 \mathrm{H}), 7.19(\mathrm{t}, J=8.0 \mathrm{~Hz}, 2 \mathrm{H}), 6.81-6.78(\mathrm{~m}, 2 \mathrm{H}), 6.67(\mathrm{~d}, J=8.0 \mathrm{~Hz}$, 
1H); ${ }^{13} \mathrm{C}$ NMR (100 MHz, DMSO) $\delta 159.92,148.59,144.46,135.59,135.31,134.45,134.21$, $134.12,130.16,129.45,129.28,128.30,127.88,127.43,127.00,126.79,125.52,124.17$, 122.39, 120.54, 119.39, 116.87, 115.96, 100.00, 61.02; HRMS (ESI-TOF) : m/z [M + H] $]^{+}$ calculated for $\mathrm{C}_{25} \mathrm{H}_{18} \mathrm{~N}_{3} \mathrm{O} 376.1449$; found 376.1450.

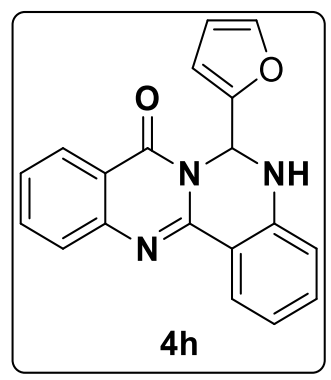

\section{6-(furan-2-yl)-5H-quinazolino[4,3-b]quinazolin-8(6H)-one (4h)}

The same general procedure was followed. Column chromatography $\left(\mathrm{SiO}_{2}\right.$, eluting with $12 \%$ ethyl acetate/pet ether) afforded the desired product as Pale yellow solid (26.75 mg, 85\% yield), mp 191-192 ${ }^{\circ} \mathrm{C}$.

${ }^{1} \mathrm{H}$ NMR $\left(600 \mathrm{MHz}, \mathrm{DMSO}-d_{6}\right) \delta=8.17-8.13(\mathrm{~m}, 2 \mathrm{H}), 7.89-7.82(\mathrm{~m}, 2 \mathrm{H}), 7.71(\mathrm{~d}, J=12$ Hz, 1H), $7.50(\mathrm{~d}, J=6.0 \mathrm{~Hz}, 2 \mathrm{H}), 7.36(\mathrm{~m}, 1 \mathrm{H}), 7.23(\mathrm{~d}, 1 \mathrm{H}), 6.94(\mathrm{~d}, J=6.0 \mathrm{~Hz}, 1 \mathrm{H}), 6.87(\mathrm{~m}$, $1 \mathrm{H}), 6.33-6.22(\mathrm{~m}, 1 \mathrm{H}), 6.09(\mathrm{~d}, J=6.0 \mathrm{~Hz}, 1 \mathrm{H}) ;{ }^{13} \mathrm{C}$ NMR $(150 \mathrm{MHz}$, DMSO-d 6$) \delta=$ $207.02,159.65,152.03,143.74,135.52$, $134.10,127.75,127.34$, 127.08 , 126.78 , $119.56,116.29,110.95,108.52$; HRMS (ESI-TOF): $\mathrm{m} / \mathrm{z}[\mathrm{M}+\mathrm{H}]^{+}$calculated for $\mathrm{C}_{19} \mathrm{H}_{14} \mathrm{~N}_{3} \mathrm{O}_{2}$ 316.1086; found 316.1087.

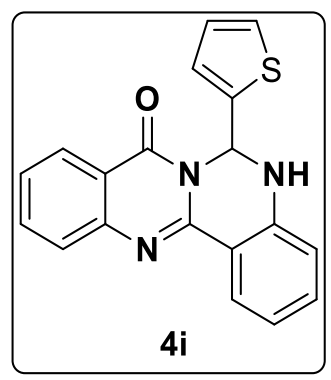

\section{6-(thiophen-2-yl)-5H-quinazolino[4,3-b]quinazolin-8(6H)-one (4i)}

The same general procedure was followed. Column chromatography $\left(\mathrm{SiO}_{2}\right.$, eluting with $12 \%$ ethyl acetate/pet ether) afforded the desired product as Pale yellow solid (27.48 $\mathrm{mg}, 83 \%$ yield), mp 195-196 ${ }^{\circ} \mathrm{C}$. 
${ }^{1} \mathrm{H}$ NMR (600 MHz, DMSO- $\left.d_{6}\right) \delta 8.17(\mathrm{dd}, J=6.0,6.0 \mathrm{~Hz}, 2 \mathrm{H}), 7.94(\mathrm{~d}, J=6.0 \mathrm{~Hz}, 1 \mathrm{H})$, $7.84(\mathrm{t}, J=6.0 \mathrm{~Hz}, 1 \mathrm{H}), 7.71(\mathrm{~d}, J=12.0 \mathrm{~Hz}, 1 \mathrm{H}), 7.50(\mathrm{t}, J=6.0 \mathrm{~Hz}, 1 \mathrm{H}), 7.45-7.37(\mathrm{~m}$, 2H), $7.30(\mathrm{~d}, J=6.0 \mathrm{~Hz}, 1 \mathrm{H}), 6.97(\mathrm{~d}, J=12.0 \mathrm{~Hz}, 1 \mathrm{H}), 6.93-6.84(\mathrm{~m}, 3 \mathrm{H}) ;{ }^{13} \mathrm{C} \mathrm{NMR}(150$ MHz, DMSO) $\delta 159.62,148.03,147.07,145.39,143.43,135.51,134.26,127.70,127.36$, 127.03, 126.99, 126.77, 126.57, 126.40, 120.36, 119.69, 116.61, 115.84, 60.30. HRMS (ESITOF) : $\mathrm{m} / \mathrm{z}[\mathrm{M}+\mathrm{H}]^{+}$calculated for $\mathrm{C}_{19} \mathrm{H}_{13} \mathrm{~N}_{3} \mathrm{OS} 332.0857$; found 332.0860 .

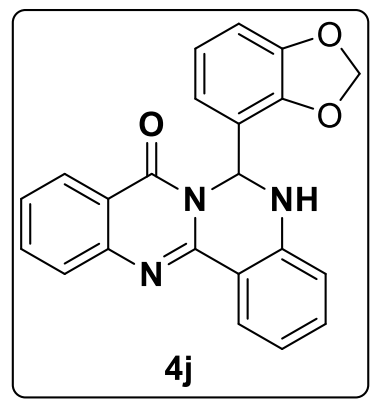

\section{6-(benzo[d][1,3]dioxol-4-yl)-5H-quinazolino[4,3-b]quinazolin-8(6H)-one (4j)}

The same general procedure was followed. Column chromatography $\left(\mathrm{SiO}_{2}\right.$, eluting with $13 \%$ ethyl acetate/pet ether) afforded the desired product as Pale yellow solid (32.48 $\mathrm{mg}, 88 \%$ yield), mp $220-221^{\circ} \mathrm{C}$.

${ }^{1} \mathrm{H}$ NMR $\left(600 \mathrm{MHz}, \mathrm{DMSO}-d_{6}\right) \delta=8.15(\mathrm{~d}, J=6.0 \mathrm{~Hz}, 2 \mathrm{H}), 7.95-7.81(\mathrm{~m}, 2 \mathrm{H}), 7.73(\mathrm{~d}$, $J=6.0 \mathrm{~Hz}, 1 \mathrm{H}), 7.50(\mathrm{t}, J=6.0 \mathrm{~Hz}, 1 \mathrm{H}), 7.33$ (t, $J=6.0 \mathrm{~Hz}, 1 \mathrm{H}), 7.15(\mathrm{~s}, 1 \mathrm{H}), 6.91$ (d, $J=12.0$ Hz, 1H), 6.82 (s, 2H), 6.73 (d, J=6.0 Hz, 1H), 6.47 (d, J=6.0 Hz, 1H), 5.93 (d, J=6.0 Hz, 2H); ${ }^{13} \mathrm{C}$ NMR (150 MHz, DMSO) $\delta=160.0,148.1,148.0,147.6,147.5,145.4,135.4,134.1$, 133.8, 127.6, 127.3, 127.1, 126.7, 120.3, 119.4, 119.2, 116.3, 115.9, 108.3, 106.6, 101.7, 62.6; HRMS (ESI-TOF) : $\mathrm{m} / \mathrm{z}[\mathrm{M}+\mathrm{H}]^{+}$calculated for $\mathrm{C}_{22} \mathrm{H}_{16} \mathrm{~N}_{3} \mathrm{O}_{3}$ 370.1191; found 370.1193 .

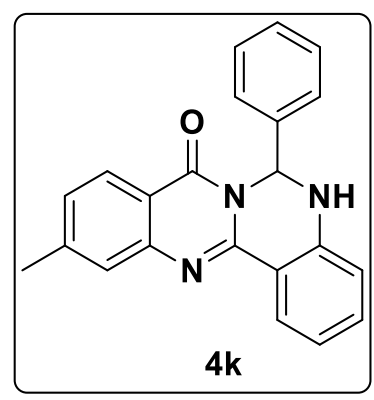

11-methyl-6-phenyl-5H-quinazolino[4,3-b]quinazolin-8(6H)-one (4k) 
The same general procedure was followed. Column chromatography $\left(\mathrm{SiO}_{2}\right.$, eluting with $12 \%$ ethyl acetate/pet ether) afforded the desired product as Pale yellow solid (25.77 $\mathrm{mg}, 76 \%$ yield), mp $222-223{ }^{\circ} \mathrm{C}$.

${ }^{1} \mathrm{H}$ NMR (600 MHz, DMSO-d6) $\delta=8.08(\mathrm{dd}, J=12.0,12.0 \mathrm{~Hz}, 1 \mathrm{H}), 7.97$ (dd, $\left.J=6.0 \mathrm{~Hz}, 2 \mathrm{H}\right)$, 7.68 (dd, $J=6.0,6.0 \mathrm{~Hz}, 1 \mathrm{H}), 7.64$ (d, $J=12.0 \mathrm{~Hz}, 1 \mathrm{H}), 7.30$ (d, J=6.0Hz, 1H), $7.26-7.20$ (m, $4 \mathrm{H}), 7.15-7.13(\mathrm{~m}, 2 \mathrm{H}), 6.91-6.89(\mathrm{~m}, 1 \mathrm{H}), 6.82-6.78(\mathrm{~m}, 1 \mathrm{H}), 2.45(\mathrm{~s}, 3 \mathrm{H}) ;{ }^{13} \mathrm{C} \mathrm{NMR}$ $\left(150 \mathrm{MHz}, \mathrm{DMSO}-d_{6}\right) \delta=146.97,146.17,145.40,139.91,136.82,133.94,128.99$, $128.94,128.58,127.60,127.55,127.16,126.42,126.10,120.08,119.23,116.31$, $116.16,62.85,21.23$; HRMS (ESI-TOF): $\mathrm{m} / \mathrm{z}[\mathrm{M}+\mathrm{H}]^{+}$calculated for $\mathrm{C}_{22} \mathrm{H}_{18} \mathrm{~N}_{3} \mathrm{O}$ 340.1449; found 340.1450.

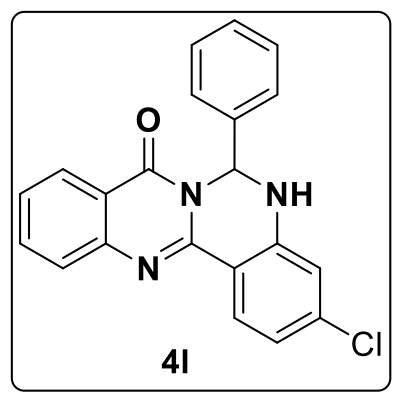

\section{3-chloro-6-phenyl-5H-quinazolino[4,3-b]quinazolin-8(6H)-one (4I)}

The same general procedure was followed. Column chromatography $\left(\mathrm{SiO}_{2}\right.$, eluting with $12 \%$ ethyl acetate/pet ether) afforded the desired product as Pale yellow solid (26.57 $\mathrm{mg}, 74 \%$ yield), mp $190-191^{\circ} \mathrm{C}$.

${ }^{1} \mathrm{H}$ NMR (600 MHz, DMSO- $\left.d_{6}\right) \delta=8.22-8.17(\mathrm{~m}, 2 \mathrm{H}), 8.11(\mathrm{~d}, J=6.0 \mathrm{~Hz}, 1 \mathrm{H}), 7.86(\mathrm{~d}$, $J=6.0 \mathrm{~Hz}, 1 \mathrm{H}), 7.73(\mathrm{~d}, J=6.0 \mathrm{~Hz}, 1 \mathrm{H}), 7.54-7.51(\mathrm{~m}, 1 \mathrm{H}), 7.28(\mathrm{~m}, 3 \mathrm{H}), 7.24(\mathrm{~d}, J=6.0 \mathrm{~Hz}$, 1H), $7.17-7.15(\mathrm{~m}, 2 \mathrm{H}), 6.99(\mathrm{~d}, J=6.0 \mathrm{~Hz}, 1 \mathrm{H}), 6.85-6.83(\mathrm{~m}, 1 \mathrm{H}) .{ }^{13} \mathrm{C} \mathrm{NMR}(150 \mathrm{MHz}$, DMSO) $\delta=159.98,147.97,146.99,146.64,139.63$, 138.63, 135.56, 129.22, 129.13, 128.78, 127.73, 127.15, 126.94, 126.04, 120.40, 119.32, 115.39, 114.74, 62.99. HRMS (ESI-TOF): $\mathrm{m} / \mathrm{z}[\mathrm{M}+\mathrm{H}]^{+}$calculated for $\mathrm{C}_{21} \mathrm{H}_{15} \mathrm{ClN}_{3} \mathrm{O} 360.0903$; found 360.0904 .

\section{General procedure for the synthesis of compounds $(5 \mathrm{a}-5 \mathrm{c})$ :}

To a solution of $1\left(0.1 \mathrm{mmol}, 1.0\right.$ equiv) in DMSO $(1 \mathrm{~mL})$, was added $\mathrm{Cu}(\mathrm{OAc})_{2} \cdot \mathrm{H}_{2} \mathrm{O}(20$ mol\%) heated at $120^{\circ} \mathrm{C}$ in a sealed tube for $24 \mathrm{~h}$. After completion of the reaction, monitored 
by TLC, the reaction mixture was diluted with ethyl acetate, filtered through Celite bed, and then washed with cold water $(4 \times 10 \mathrm{~mL})$ and brine $(2 \times 10 \mathrm{~mL})$. The organic layer was then collected and dried over $\mathrm{Na}_{2} \mathrm{SO}_{4}$. After that, the organic extract was evaporated under reduced pressure to get crude solid. It was then subjected to column chromatography (silica gel 230-400 mesh size, ethyl acetate: pet ether) for further purification to get the desired compounds $(5 \mathrm{a}-5 \mathrm{c})$ in $80-92 \%$ yield.

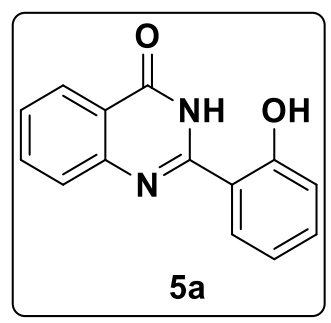

\section{2-(2-hydroxyphenyl)quinazolin-4(3H)-one (5a):}

The same general procedure was followed. Column chromatography $\left(\mathrm{SiO}_{2}\right.$, eluting with $15 \%$ ethyl acetate/pet ether) afforded the desired product as white solid (21.09 $\mathrm{mg}, 92 \%$ yield), $\mathrm{mp}$ $180-181^{\circ} \mathrm{C}$.

${ }^{1} \mathrm{H}$ NMR (600 MHz, DMSO-d6) $\delta 13.78(\mathrm{~s}, 1 \mathrm{H}), 12.49(\mathrm{~s}, 1 \mathrm{H}), 8.22(\mathrm{~d}, J=12.0 \mathrm{~Hz}, 1 \mathrm{H})$, $8.15(\mathrm{~d}, J=6.0 \mathrm{~Hz}, 1 \mathrm{H}), 7.85(\mathrm{t}, J=6.0 \mathrm{~Hz}, 1 \mathrm{H}), 7.76(\mathrm{~d}, J=12.0 \mathrm{~Hz}, 1 \mathrm{H}), 7.54(\mathrm{t}, J=6.0$ $\mathrm{Hz}, 1 \mathrm{H}), 7.44(\mathrm{t}, J=6.0 \mathrm{~Hz}, 1 \mathrm{H}), 7.00(\mathrm{~d}, J=12.0 \mathrm{~Hz}, 1 \mathrm{H}), 6.95(\mathrm{t}, J=6.0 \mathrm{~Hz}, 1 \mathrm{H}) ;{ }^{13} \mathrm{C}$ NMR (150 MHz, DMSO) $\delta 161.92,160.55,154.26,146.61,135.51,134.21,128.18,127.44$, 126.53, 121.21, 119.30, 118.38, 114.23; HRMS (ESI-TOF) : $\mathrm{m} / \mathrm{z}[\mathrm{M}+\mathrm{H}]^{+}$calculated for $\mathrm{C}_{14} \mathrm{H}_{10} \mathrm{~N}_{2} \mathrm{O}_{2} 239.0815$; found 239.0824.

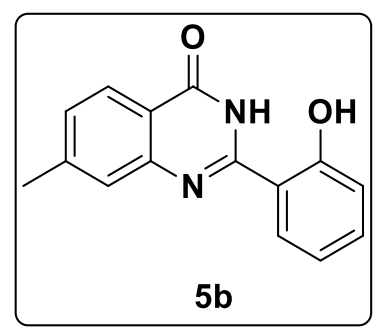

\section{2-(2-hydroxyphenyl)-7-methylquinazolin-4(3H)-one (5b):}

The same general procedure was followed. Column chromatography $\left(\mathrm{SiO}_{2}\right.$, eluting with $15 \%$ ethyl acetate/pet ether) afforded the desired product as white solid (22.61 $\mathrm{mg}, 89 \%$ yield), $\mathrm{mp}$ $178-179^{\circ} \mathrm{C}$. 
${ }^{1} \mathrm{H}$ NMR (600 MHz, DMSO-d $\left.d_{6}\right) \delta=13.82(\mathrm{~s}, 1 \mathrm{H}), 12.42(\mathrm{~s}, 1 \mathrm{H}), 8.20$ (dd, J=6.0, $6.0 \mathrm{~Hz}$, 1H), $7.95(\mathrm{~s}, 1 \mathrm{H}), 7.70-7.65(\mathrm{~m}, 2 \mathrm{H}), 7.46-7.40(\mathrm{~m}, 1 \mathrm{H}), 6.99(\mathrm{~d}, J=6.0 \mathrm{~Hz}, 1 \mathrm{H}), 6.95$ (d, $J=6.0 \mathrm{~Hz}, 1 \mathrm{H}), 2.45$ (s, 3H). ${ }^{13} \mathrm{C}$ NMR (150 MHz, DMSO) $\delta=161.81,160.42,153.39$, 144.52 , 137.35, 136.79, 134.03, 128.00, 126.41, 125.93, 120.97, 119.29, 118.33, 114.19, 21.29. HRMS (ESI-TOF): $\mathrm{m} / \mathrm{z}[\mathrm{M}+\mathrm{H}]^{+}$calculated for $\mathrm{C}_{15} \mathrm{H}_{13} \mathrm{~N}_{2} \mathrm{O}_{2}$ 253.0977; found 253.0979.

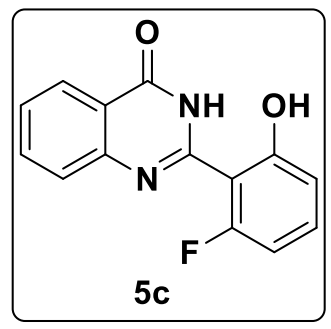

\section{2-(2-fluoro-6-hydroxyphenyl)quinazolin-4(3H)-one (5c):}

The same general procedure was followed. Column chromatography $\left(\mathrm{SiO}_{2}\right.$, eluting with $15 \%$ ethyl acetate/pet ether) afforded the desired product as white solid (20.48 mg, 80\% yield), mp $190-191^{\circ} \mathrm{C}$.

${ }^{1} \mathrm{H}$ NMR (600 MHz, DMSO-d $)_{6} \delta 12.44(\mathrm{~s}, 1 \mathrm{H}), 10.64(\mathrm{~s}, 1 \mathrm{H}), 8.15$ (d, $\left.J=6.0 \mathrm{~Hz}, 1 \mathrm{H}\right), 7.83$ $(\mathrm{t}, J=6.0 \mathrm{~Hz}, 1 \mathrm{H}), 7.68(\mathrm{~d}, J=6.0 \mathrm{~Hz}, 1 \mathrm{H}), 7.55(\mathrm{t}, J=12.0 \mathrm{~Hz}, 1 \mathrm{H}), 7.34(\mathrm{q}, J=6.0 \mathrm{~Hz}$, $1 \mathrm{H}), 6.81-6.75(\mathrm{~m}, 2 \mathrm{H}) ;{ }^{13} \mathrm{C}$ NMR $(150 \mathrm{MHz}, \mathrm{DMSO}) \delta 162.01,160.75\left(\mathrm{~d},{ }^{1} J_{\mathrm{C}-\mathrm{F}}=244.5\right.$ $\mathrm{Hz}), 157.69$ (d, $\left.{ }^{3} J_{\mathrm{C}-\mathrm{F}}=7.5 \mathrm{~Hz}\right), 149.00,148.73,135.00,132.33,127.69,127.45,126.29$, 121.70, 112.53, 111.22, $106.17\left(\mathrm{~d},{ }^{2} J_{\mathrm{C}-\mathrm{F}}=21 \mathrm{~Hz}\right) ;$ HRMS (ESI-TOF): $\mathrm{m} / \mathrm{z}[\mathrm{M}+\mathrm{H}]^{+}$ calculated for $\mathrm{C}_{14} \mathrm{H}_{9} \mathrm{FN}_{2} \mathrm{O}_{2} 257.0720$; found 257.0734

\section{X-ray Crystallography Data}

\section{CCDC1954068}

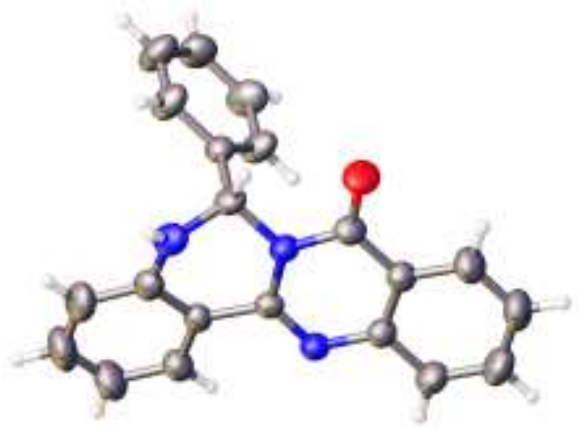

X-ray crystal structure of $\mathbf{4 a}$ 
Table S1: Important crystal data of product 4a.

Empirical Formula

Formula weight

Temperature

Wave length

Crystal system

Space group

Unit cell dimensions

Volume

Z

Density (calculated)

Absorption coefficient $(\mathrm{Mu})$

$\mathrm{F}(000)$

Theta range for data collection

Index ranges

Reflection collected

Independent reflection

Absorption correction

Data/restraints/parameters

Good of fit on $\left(\mathrm{F}^{2}\right)$

Final $\mathrm{R}$ indices $[\mathrm{I}>=2 \sigma(\mathrm{I})]$

$\mathrm{R}$ indices (all data)
$\mathrm{C}_{21} \mathrm{H}_{15} \mathrm{~N}_{3} \mathrm{O}$

325.36

$273 \mathrm{~K}$

0.71073

Monoclinic

P 1 21/n 1

$a=12.6538(10) \AA \quad \alpha=90$

$\mathrm{b}=8.5038(6) \AA \quad \beta=96.896(3)$

$\mathrm{c}=15.2787(11) \AA \gamma=90$

1632.2(2) $\AA^{3}$

4

$1.324 \mathrm{~g} / \mathrm{cm}^{3}$

$0.084 \mathrm{~mm}^{-1}$

680.0

$2.89^{\circ}$ to $21.98^{\circ}$

$-16 \leq \mathrm{h} \leq 16,-10 \leq \mathrm{k} \leq 11,-19 \leq 1 \leq 19$

26612

$3692\left[R_{\text {int }}=0.0415, R_{\text {sigma }}=0.0226\right]$

multi-scan

$3692 / 0 / 226$

1.043

$\mathrm{R}_{1}=0.0515, \mathrm{wR}_{2}=0.1434$

$\mathrm{R}_{1}=0.0608, \mathrm{wR}_{2}=0.1547$

\section{CCDC1954069}

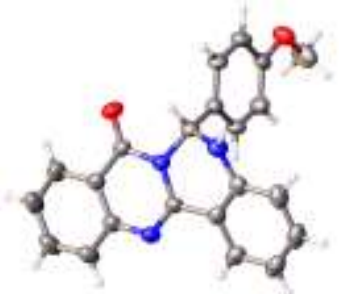

X-ray crystal structure of $\mathbf{4 f}$ 
Table S2: Important crystal data of product $4 \mathbf{f}$.

Empirical Formula

Formula weight

Temperature

Wave length

Crystal system

Space group

Unit cell dimensions

Volume

$\mathrm{Z}$

Density (calculated)

Absorption coefficient $(\mathrm{Mu})$

$\mathrm{F}(000)$

Theta range for data collection

Index ranges

Reflection collected

Independent reflection

Absorption correction

Data/restraints/parameters

Good of fit on $\left(\mathrm{F}^{2}\right)$

Final $\mathrm{R}$ indices $[\mathrm{I}>=2 \sigma(\mathrm{I})]$

$\mathrm{R}$ indices (all data)
$\mathrm{C}_{22} \mathrm{H}_{17} \mathrm{~N}_{3} \mathrm{O}_{2}$

355.38

$296 \mathrm{~K}$

0.71073

Monoclinic

P 1211

$a=6.3236(11) \AA \quad \alpha=90$

$\mathrm{b}=11.845(2) \AA \beta=104.121(2)$

$\mathrm{c}=11.642(2) \AA \gamma=90$

845.7(3) $\AA^{3}$

2

$1.396 \mathrm{~g} / \mathrm{cm}^{3}$

$0.092 \mathrm{~mm}^{-1}$

372.0

$2.49^{\circ}$ to $27.05^{\circ}$

$-7 \leq \mathrm{h} \leq 7,-14 \leq \mathrm{k} \leq 14,-13 \leq 1 \leq 13$

9601

$2950\left[R_{\text {int }}=0.0436, R_{\text {sigma }}=0.0361\right]$

multi-scan

$2950 / 1 / 246$

1.223

$\mathrm{R}_{1}=0.0483, \mathrm{wR}_{2}=0.1124$

$\mathrm{R}_{1}=0.0486, \mathrm{wR}_{2}=0.1129$

\section{CCDC1954063}




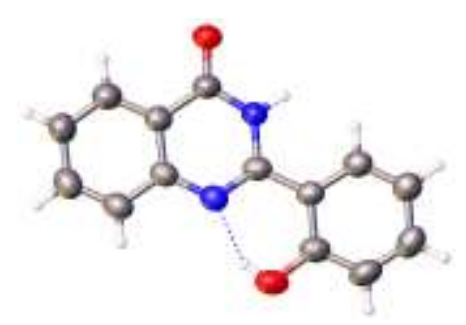

X-ray crystal structure of $\mathbf{5 a}$

Table S3: Important crystal data of product 5a.

Empirical Formula

Formula weight

Temperature

Wave length

Crystal system

Space group

Unit cell dimensions

Volume

Z

Density (calculated)

Absorption coefficient (Mu)

$\mathrm{F}(000)$

Theta range for data collection

Index ranges

Reflection collected

Independent reflection

Absorption correction

Data/restraints/parameters

Good of fit on $\left(\mathrm{F}^{2}\right)$

Final $\mathrm{R}$ indices $[\mathrm{I}>=2 \sigma(\mathrm{I})]$

$\mathrm{R}$ indices (all data)
$\mathrm{C}_{14} \mathrm{H}_{10} \mathrm{~N}_{2} \mathrm{O}_{2}$

238.25

$296 \mathrm{~K}$

0.71073

Monoclinic

P 1 21/n 1

$a=13.354(12) \AA \quad \alpha=90$

$\mathrm{b}=5.119(5) \AA \beta=101.499(13)$

$\mathrm{c}=16.699(15) \AA \gamma=90$

1118.6(17) $\AA^{3}$

4

$1.414 \mathrm{~g} / \mathrm{cm}^{3}$

$0.097 \mathrm{~mm}^{-1}$

496.2

$2.49^{\circ}$ to $21.87^{\circ}$

$-15 \leq \mathrm{h} \leq 15,-6 \leq \mathrm{k} \leq 6,-18 \leq 1 \leq 20$

9574

$1891\left[\mathrm{R}_{\text {int }}=0.0680, \mathrm{R}_{\text {sigma }}=0.0689\right]$

multi-scan

$1891 / 0 / 165$

0.997

$\mathrm{R}_{1}=0.0524, \mathrm{wR}_{2}=0.1319$

$\mathrm{R}_{1}=0.1278, \mathrm{wR}_{2}=0.1662$ 


\section{DFT studies for Compound 4a:}

\section{Computational Details:}

Quantum chemical calculations were performed using the Gaussian 09, Revision E.01 $\operatorname{program}^{1}$. The calculations were carried out for Compound 4a derivative with the density functional theory using Becke's three-parameter hybrid functional with correlation formula of Lee, Yang, and Parr (B3LYP). ${ }^{2,3}$ The compounds were fully optimized with 6-31+G (d,p) basis set, and harmonic vibrational frequency calculations were used to confirm that the optimized structures were minima, as characterized by positive vibrational frequencies. ${ }^{4,5}$<smiles>O=c1c2ccccc2nc2n1C(c1ccccc1)Nc1ccccc1-2</smiles>

4a

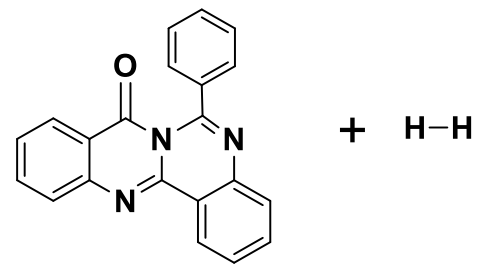

$4 a^{\prime}$

Scheme S19: Free energy diffrence (in $\mathrm{kcal} \mathrm{mol}^{-1}$ ) obtained using B3LYP/6-31+G $(\mathrm{d}, \mathrm{p})$ in gaseous phase. 


\section{References}

1. Frisch, M. J.; Trucks, G. W.; Schlegel, H. B.; Scuseria, G. E.; Robb, M. A.; Cheeseman, J. R.; Montgomery, J. A., Jr.; Vreven, T.; Kudin, K. N.; Burant, J. C.; Millam, J. M.; Iyengar, S. S.; Tomasi, J.; Barone, V.; Mennucci, B.; Cossi, M.; Scalmani, G.; Rega, N.; Petersson, G. A.; Nakatsuji, H.; Hada, M.; Ehara, M.; Toyota, K.; Fukuda, R.; Hasegawa, J.; Ishida, M.; Nakajima, T.; Honda, Y.; Kitao, O.; Nakai, H.; Klene, M.; Li, X.; Knox, J. E.; Hratchian, H. P.; Cross, J. B.; Bakken, V.; Adamo, C.; Jaramillo, J.; Gomperts, R.; Stratmann, R. E.; Yazyev, O.; Austin, A. J.; Cammi, R.; Pomelli, C.; Ochterski, J. W.; Ayala, P. Y.; Morokuma, K.; Voth, G. A.; Salvador, P.; Dannenberg, J. J.; Zakrzewski, V. G.; Dapprich, S.; Daniels, A. D.; Strain, M. C.; Farkas, O.; Malick, D. K.; Rabuck, A. D.; Raghavachari, K.; Foresman, J. B.; Ortiz, J. V.; Cui, Q.; Baboul, A. G.; Clifford, S.; Cioslowski, J.; Stefanov, B. B.; Liu, G.; Liashenko, A.; Piskorz, P.; Komaromi, I.; Martin, R. L.; Fox, D. J.; Keith, T.; Al-Laham, M. A.; Peng, C. Y.; Nanayakkara, A.; Challacombe, M.; Gill, P. M. W.; Johnson, B.; Chen, W.; Wong, M. W.; Gonzalez, C.; Pople, J. A. Gaussian 03, Gaussian Inc: Pittsburgh, PA, 2003.

2. A. D. Becke, J. Chem. Phys., 1993, 98, 5648-5652.

3. C. Lee.; W. Yang.; R. G. Parr. Phys. Rev. B, 1988, 37, 785-789

4. I. A. Koppel.; R. Schwesinger.; T. Breuer.; P. Burk.; K. Herodes.; I. Koppel.; I. Leito.; M. Mishima.; J. Phys. Chem. A. 2001, 105, 9575- 9586.

5. P. Burk.; I. A. Koppel.; I. Koppel.; I. Leito.; O. Travnikova. Chem. Phys. Lett. 2ooo, 323, 482-489. 
8. ${ }^{1} \mathrm{H}$ and ${ }^{13} \mathrm{C}$ Spectra:

${ }^{1} \mathrm{H}$ NMR of Compound 3a:

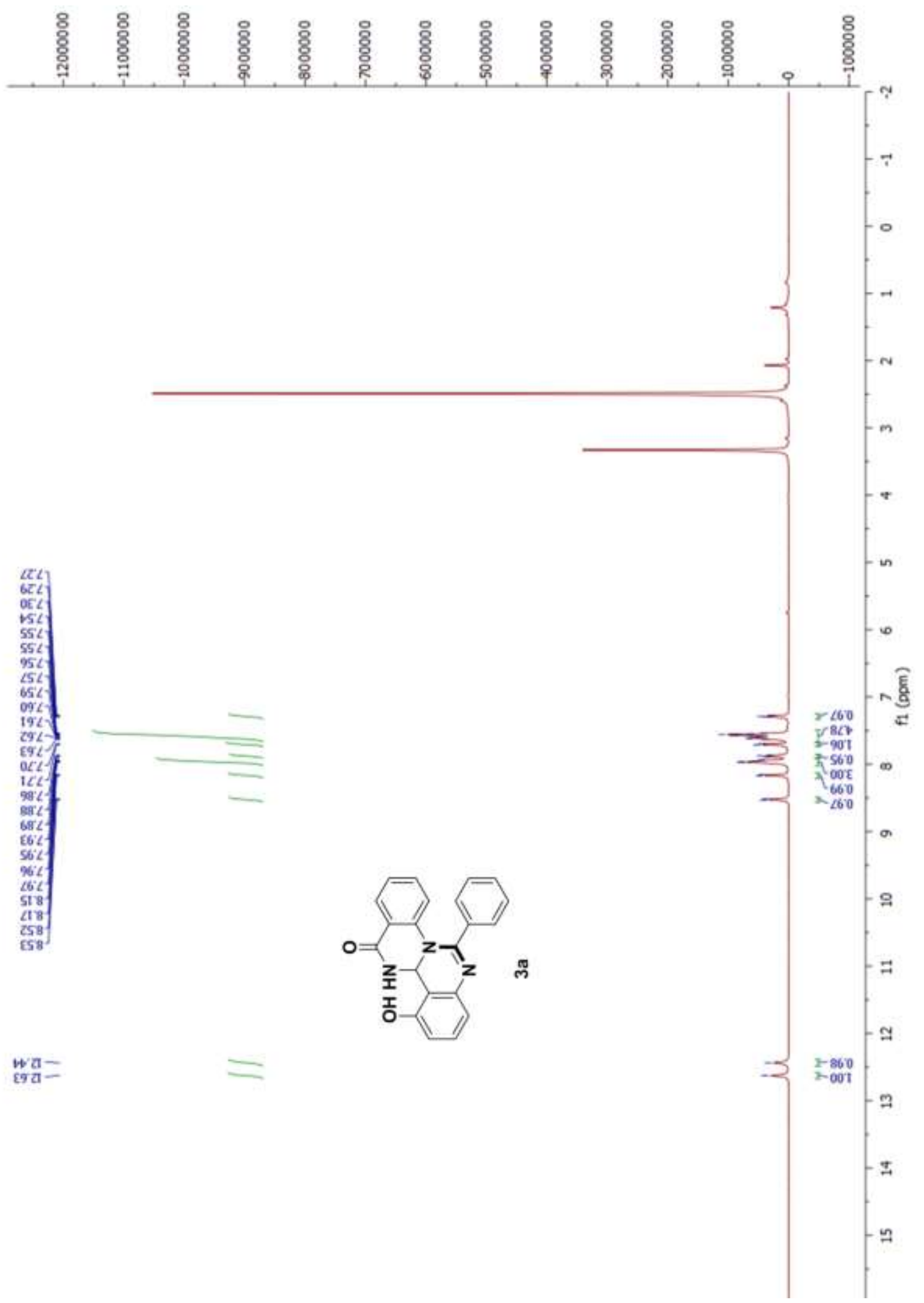


${ }^{13} \mathrm{C}$ NMR of Compound 3a:

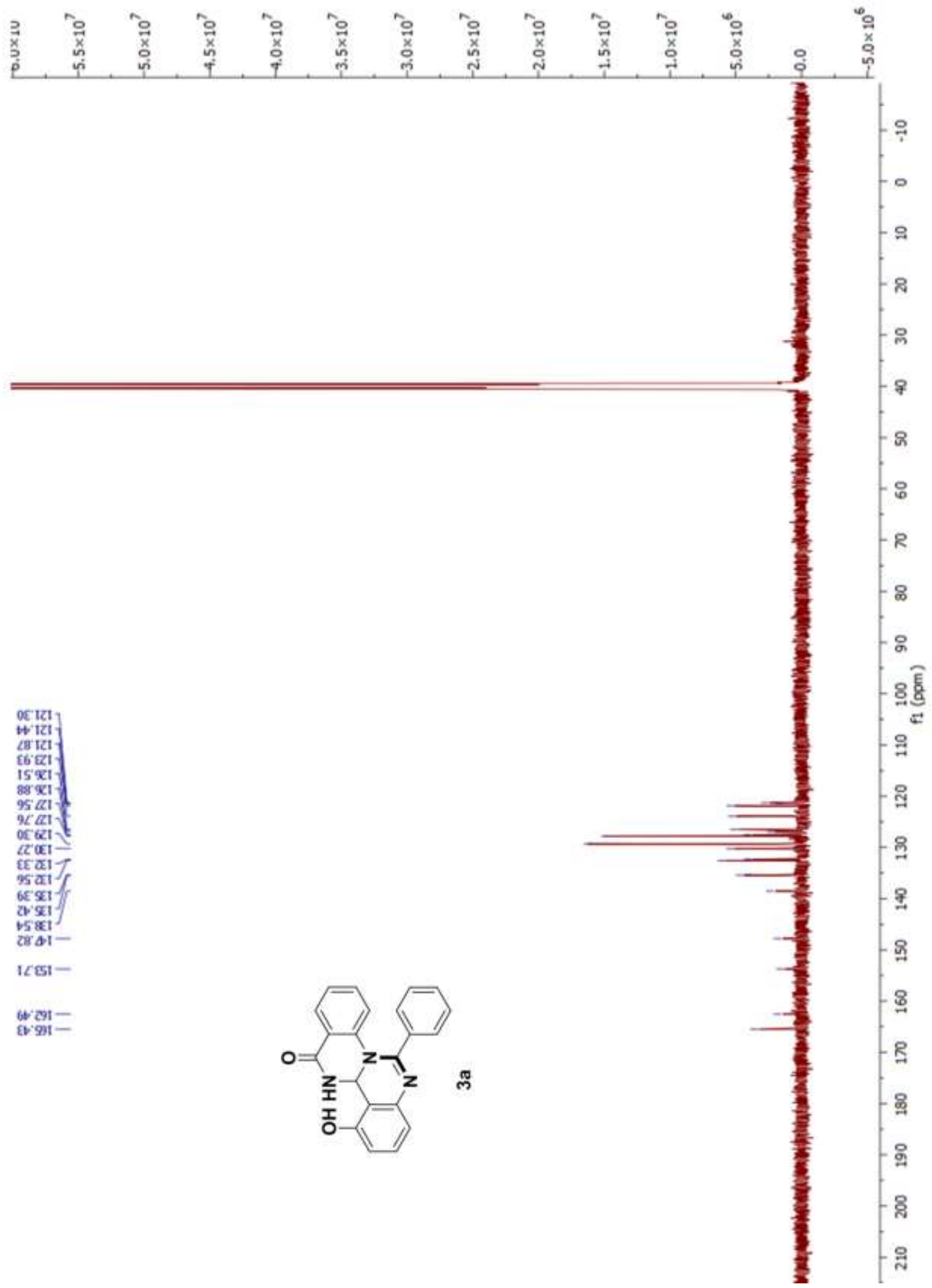


${ }^{1} \mathrm{H}$ NMR of Compound 3b:

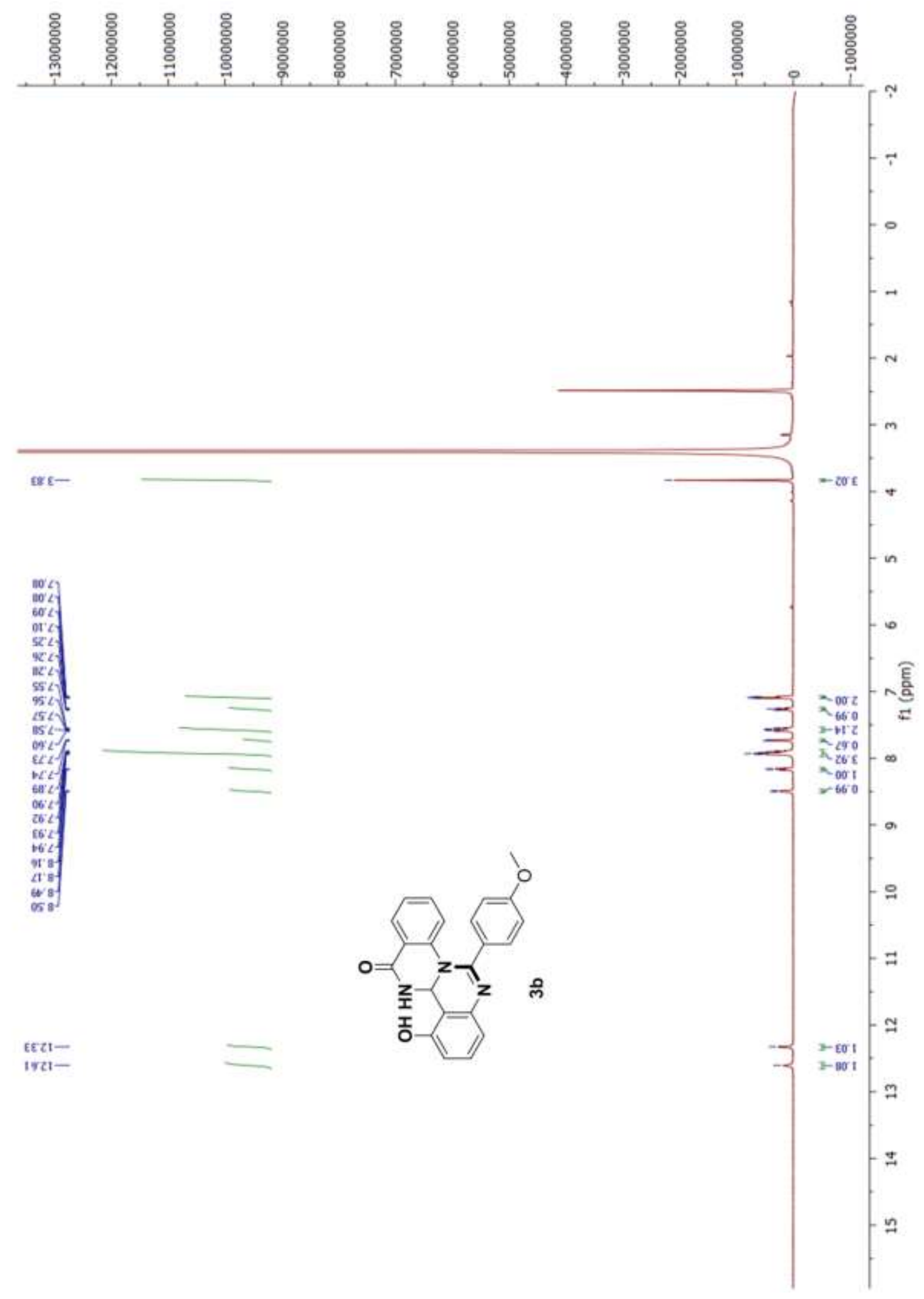


${ }^{13} \mathrm{C}$ NMR of Compound 3b:

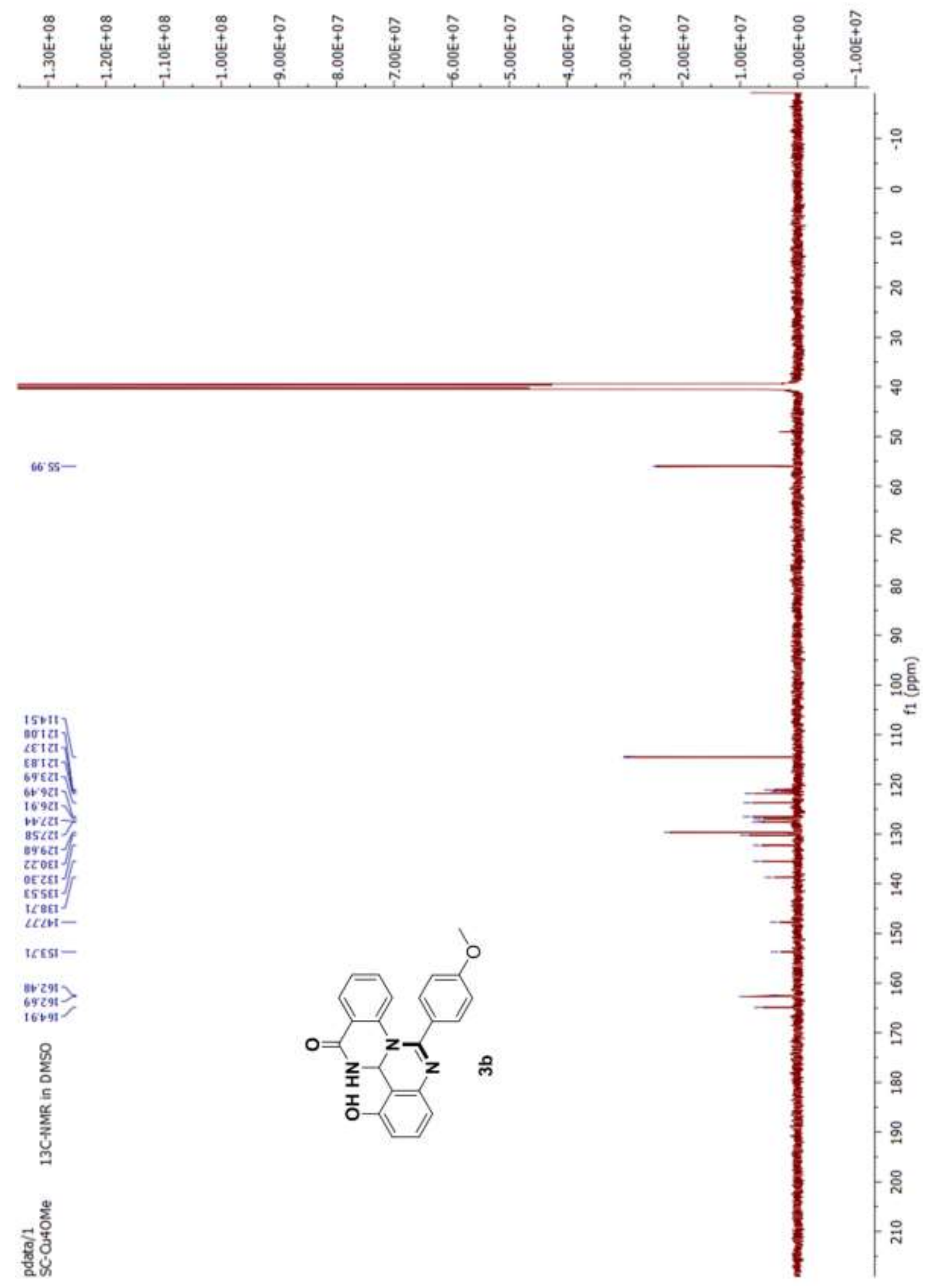


${ }^{1} \mathrm{H}$ NMR of Compound 3c:

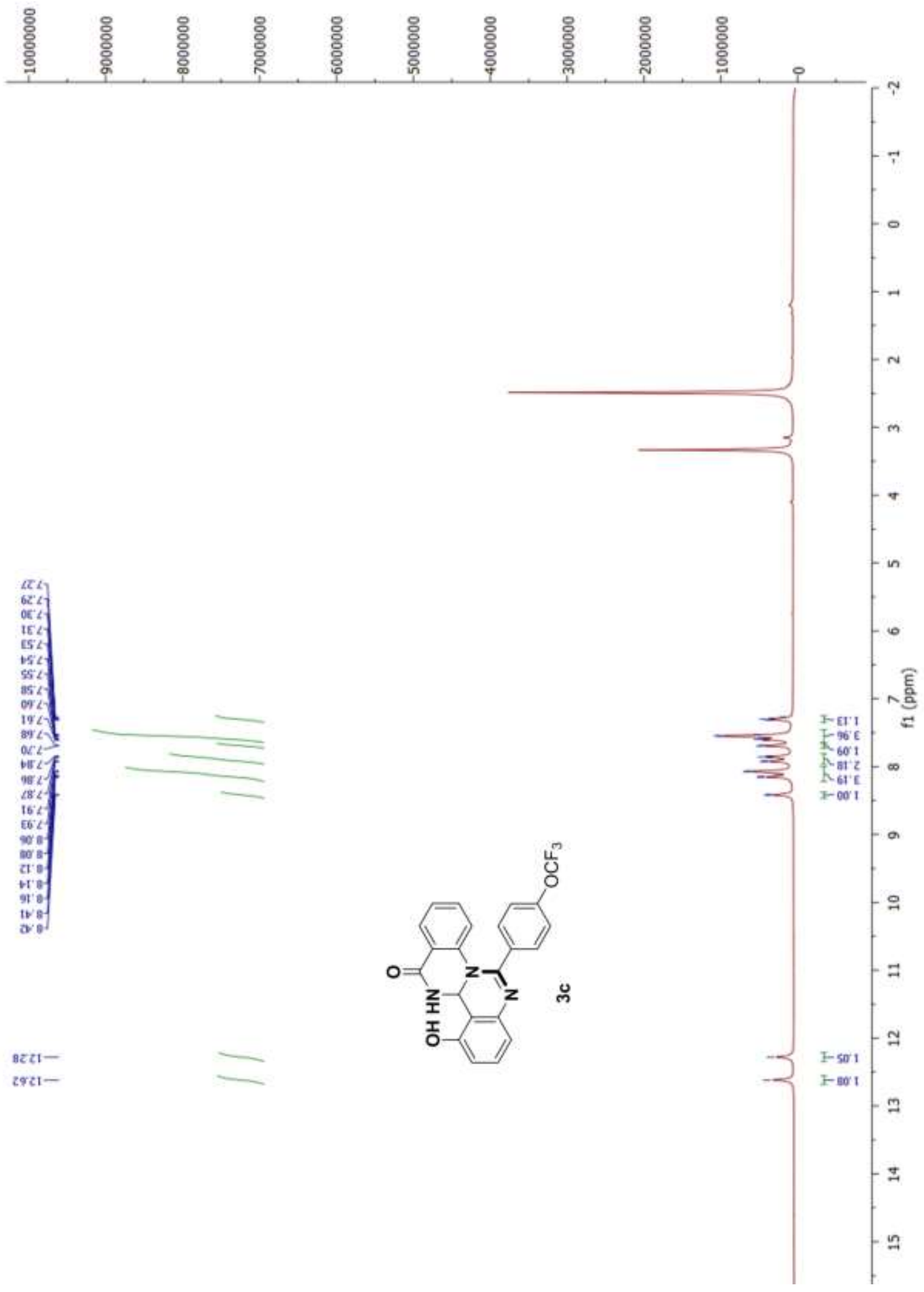


${ }^{13} \mathrm{C}$ NMR of Compound 3c:

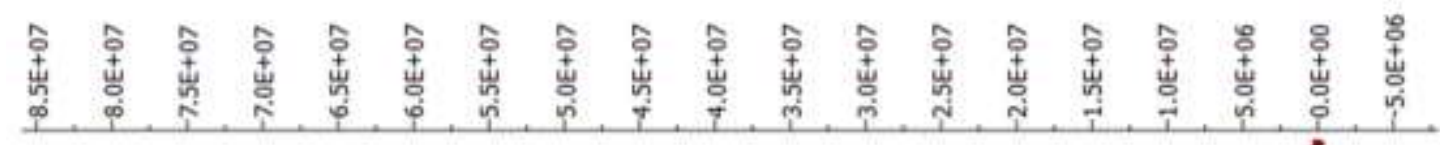

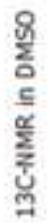

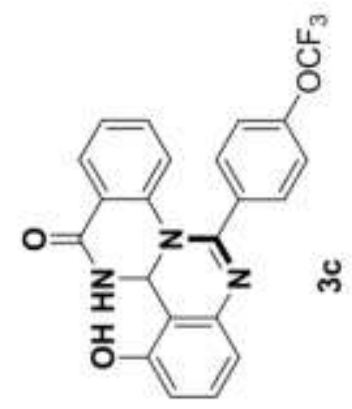

$$
\text { , }, \vec{i}, \vec{i}, \text {, }
$$

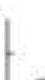


ROESY Spectra of Compound 3c:

(udd) it

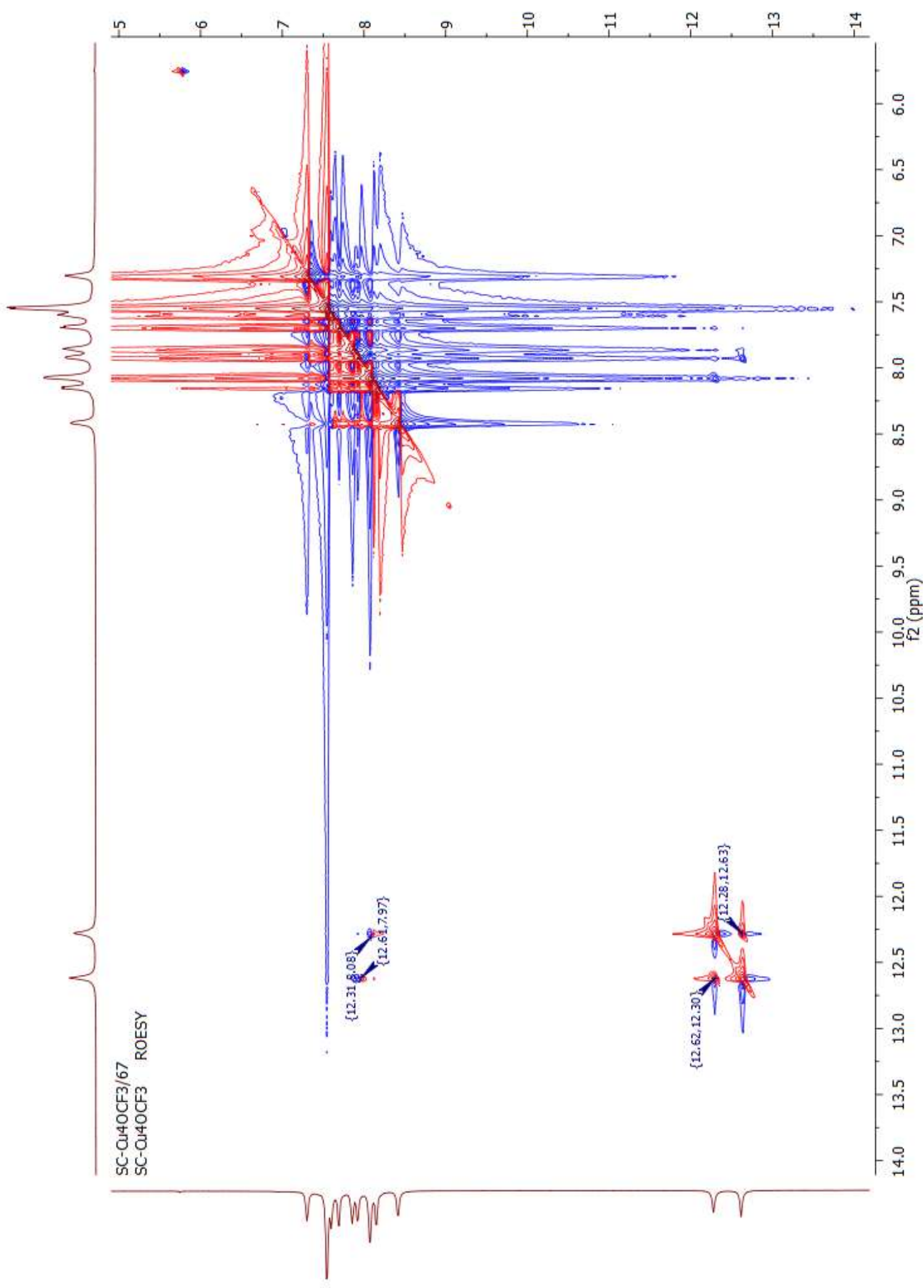


${ }^{1} \mathrm{H}$ NMR of Compound 3d:

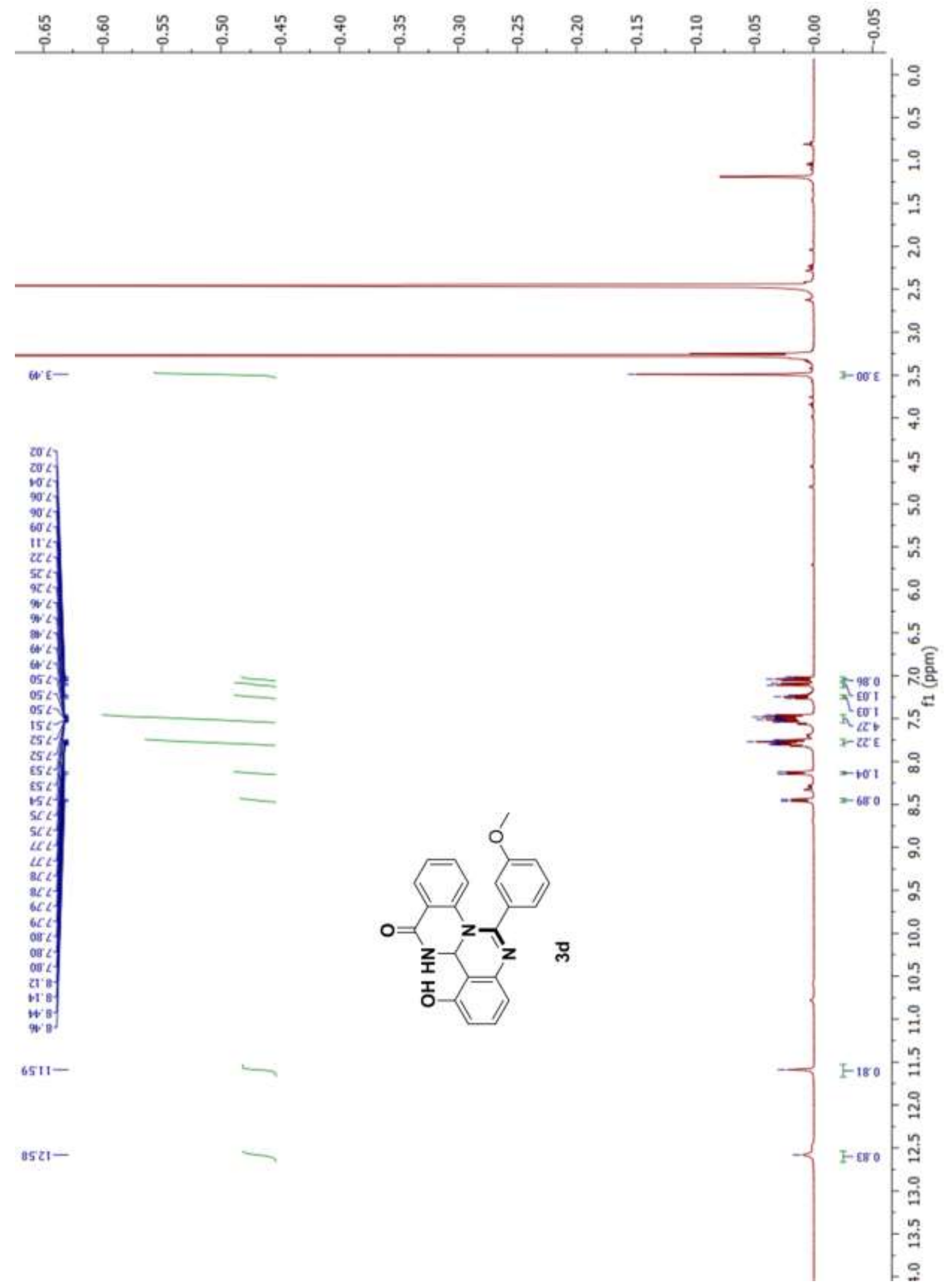


${ }^{13}$ C NMR of Compound 3d:

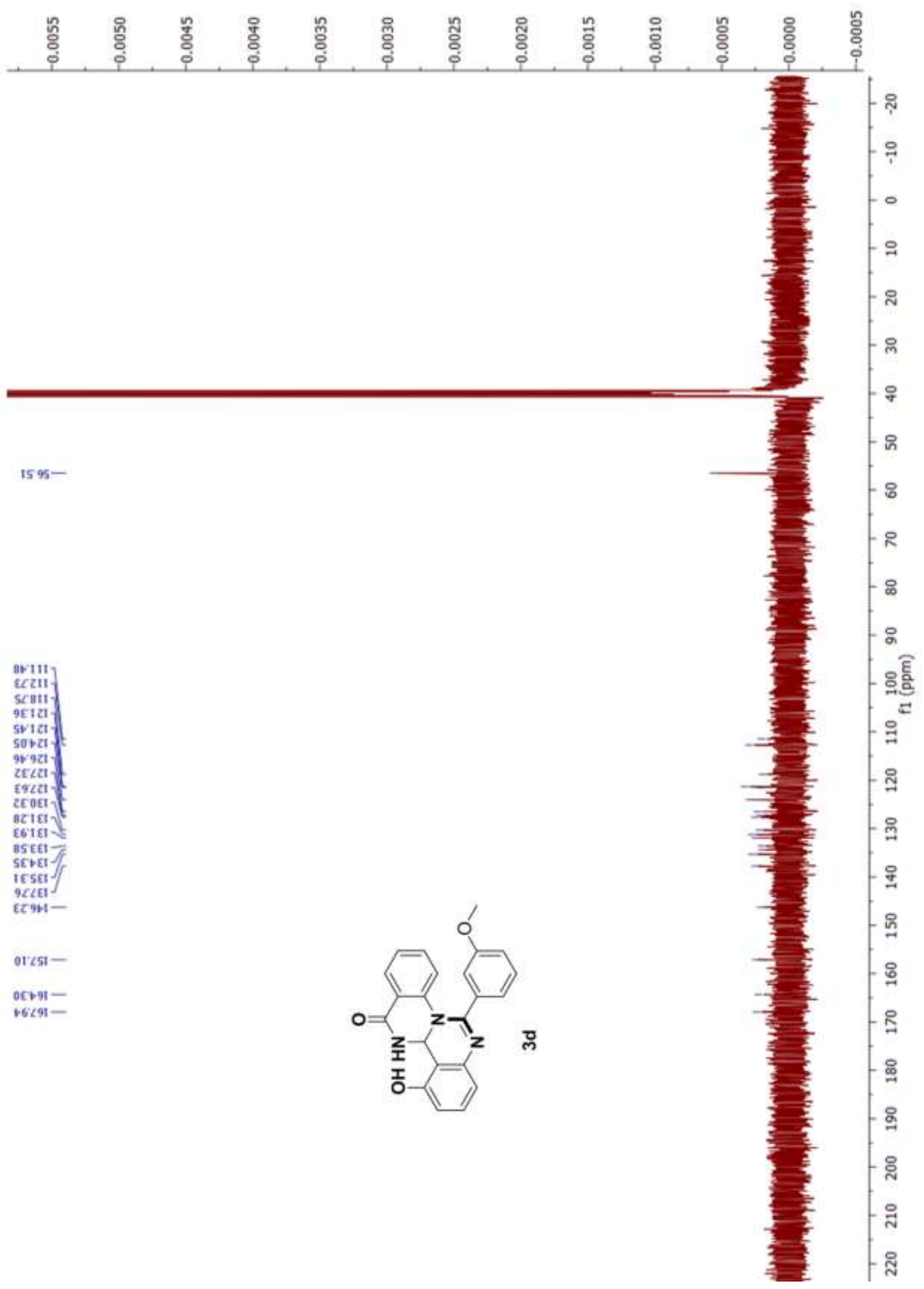

S31 
${ }^{1} \mathrm{H}$ NMR of Compound 3e:

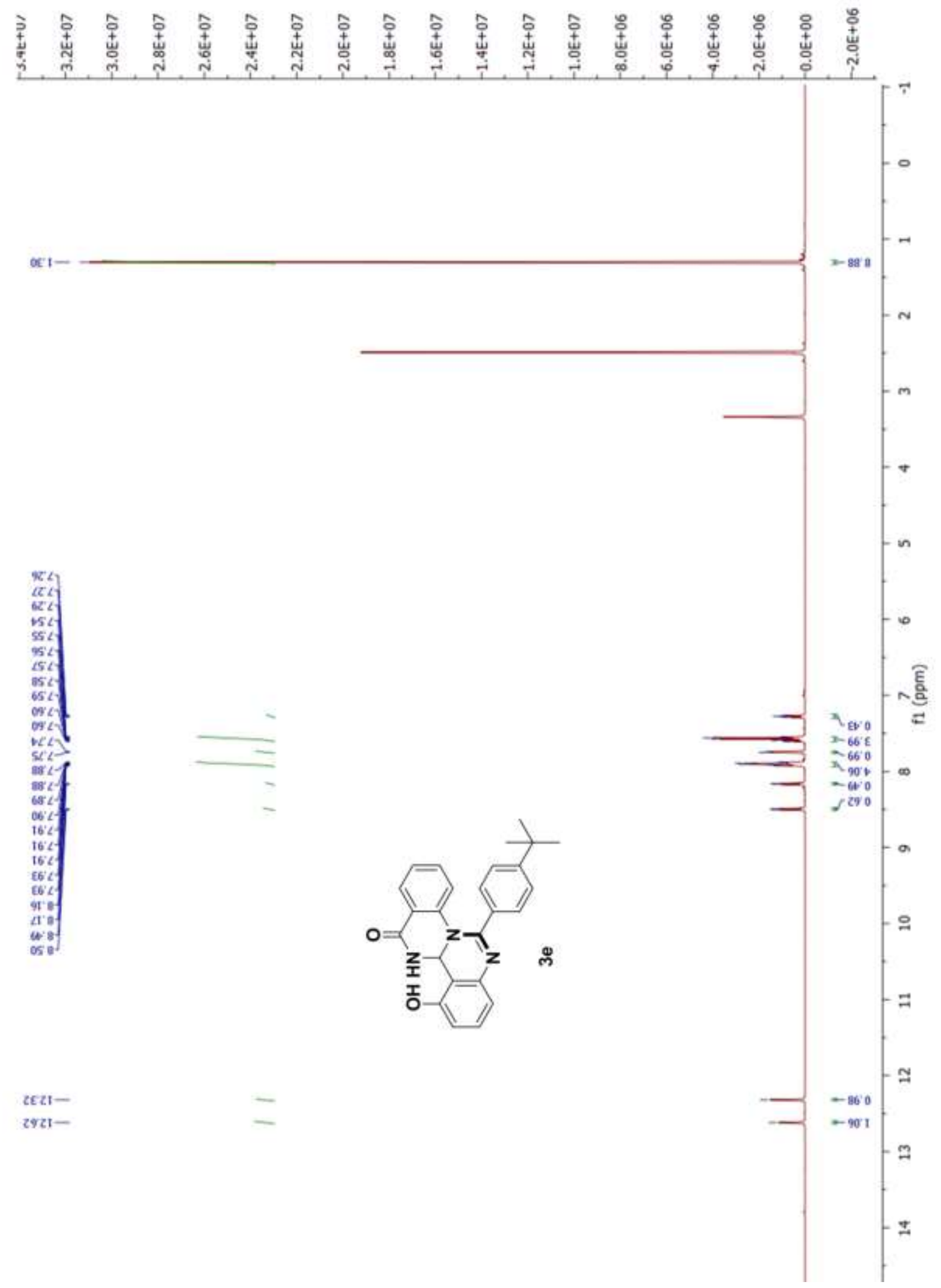


${ }^{13} \mathrm{C}$ NMR of Compound 3e:
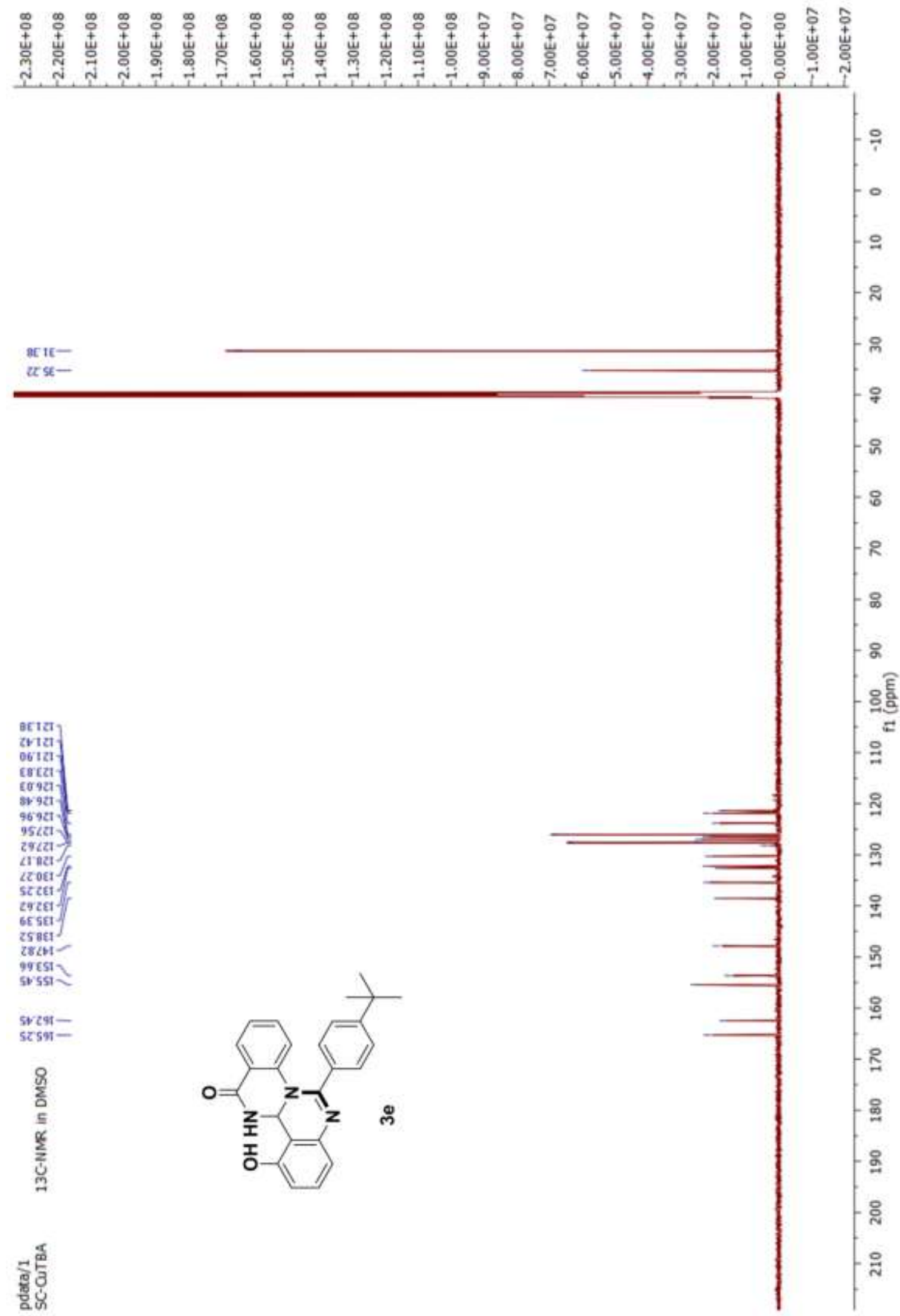
${ }^{1} \mathrm{H}$ NMR of Compound 3f:

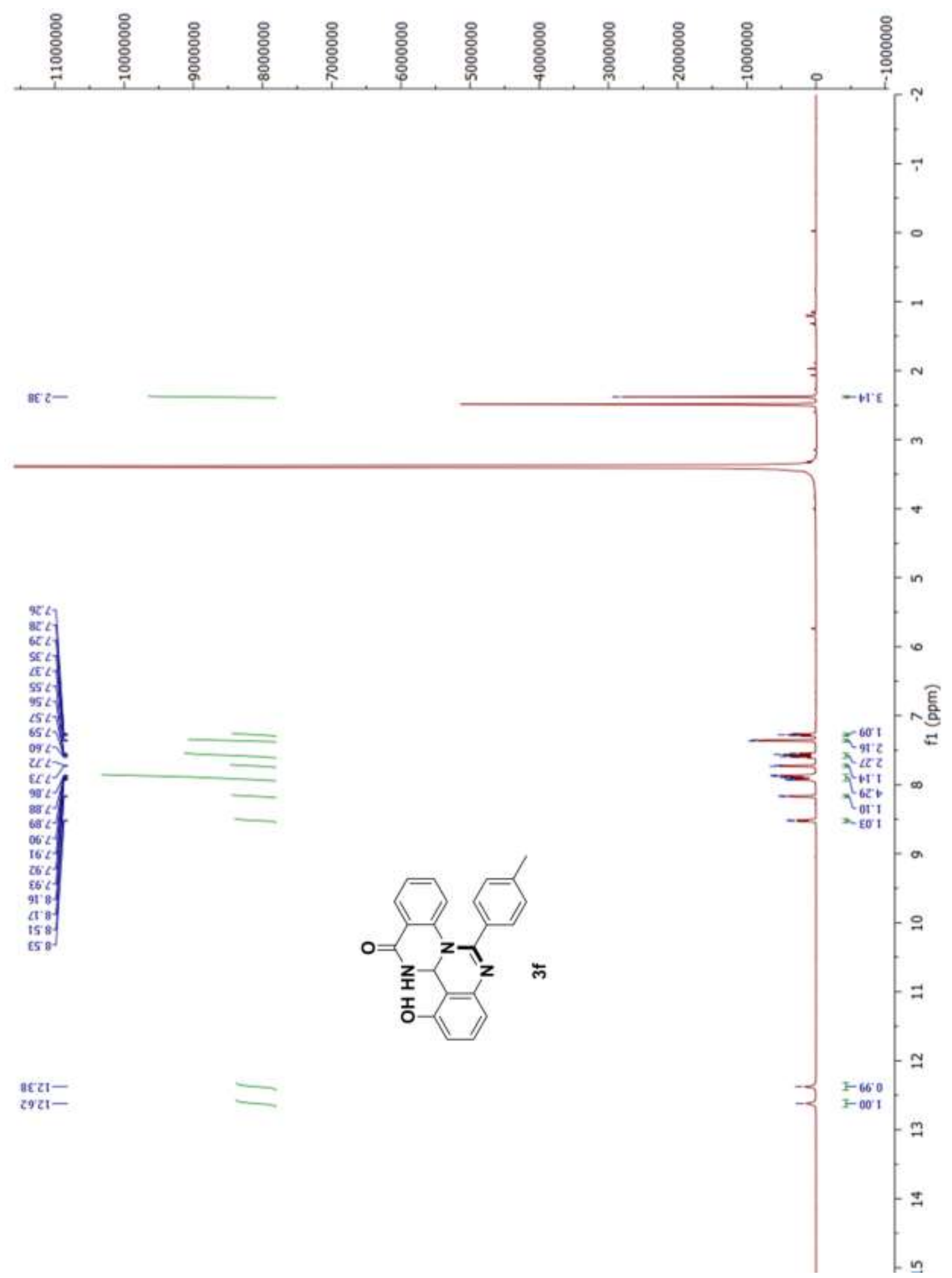


${ }^{13}$ C NMR of Compound 3f:

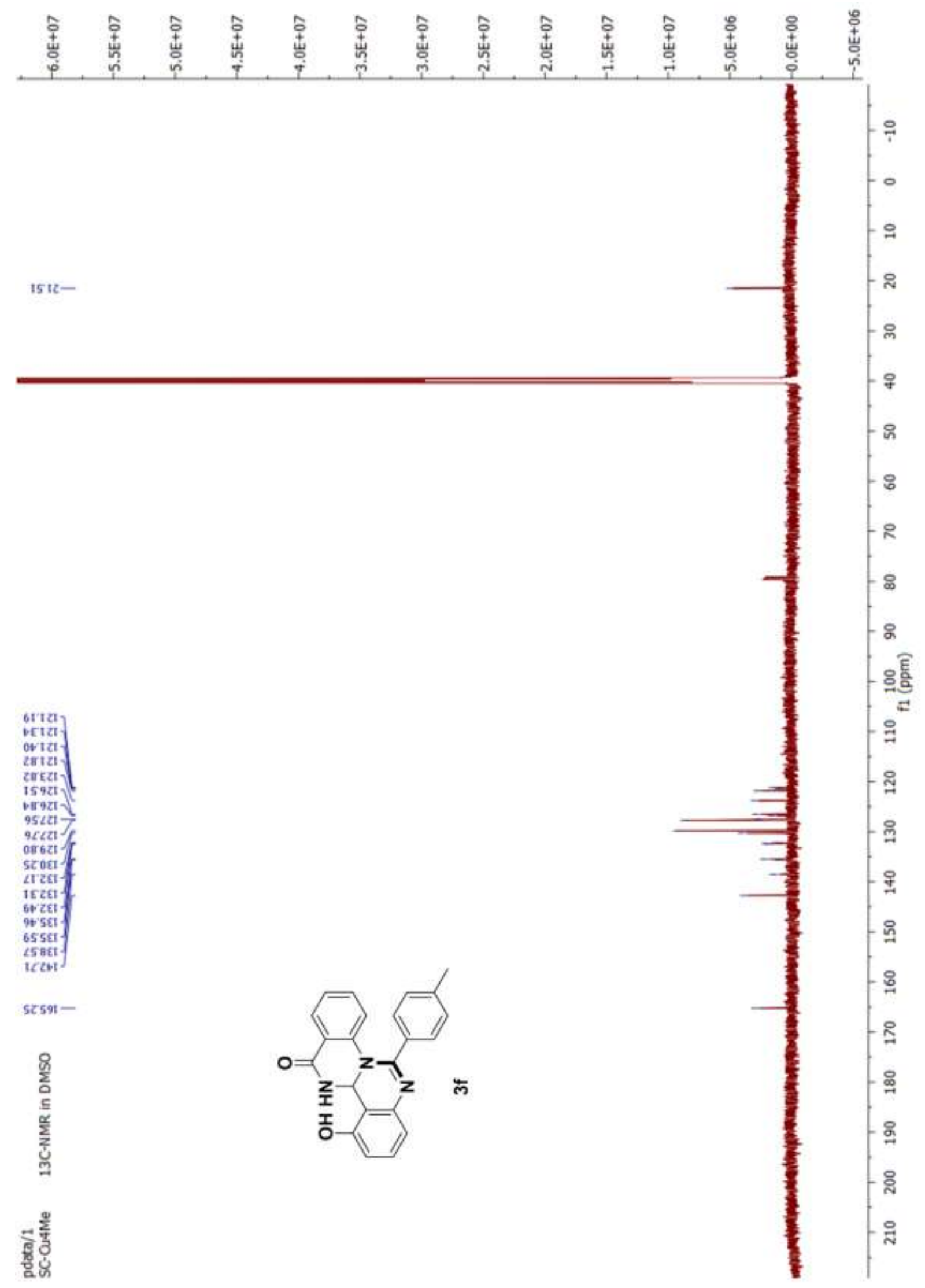


${ }^{1} \mathrm{H}$ NMR of Compound 3g:

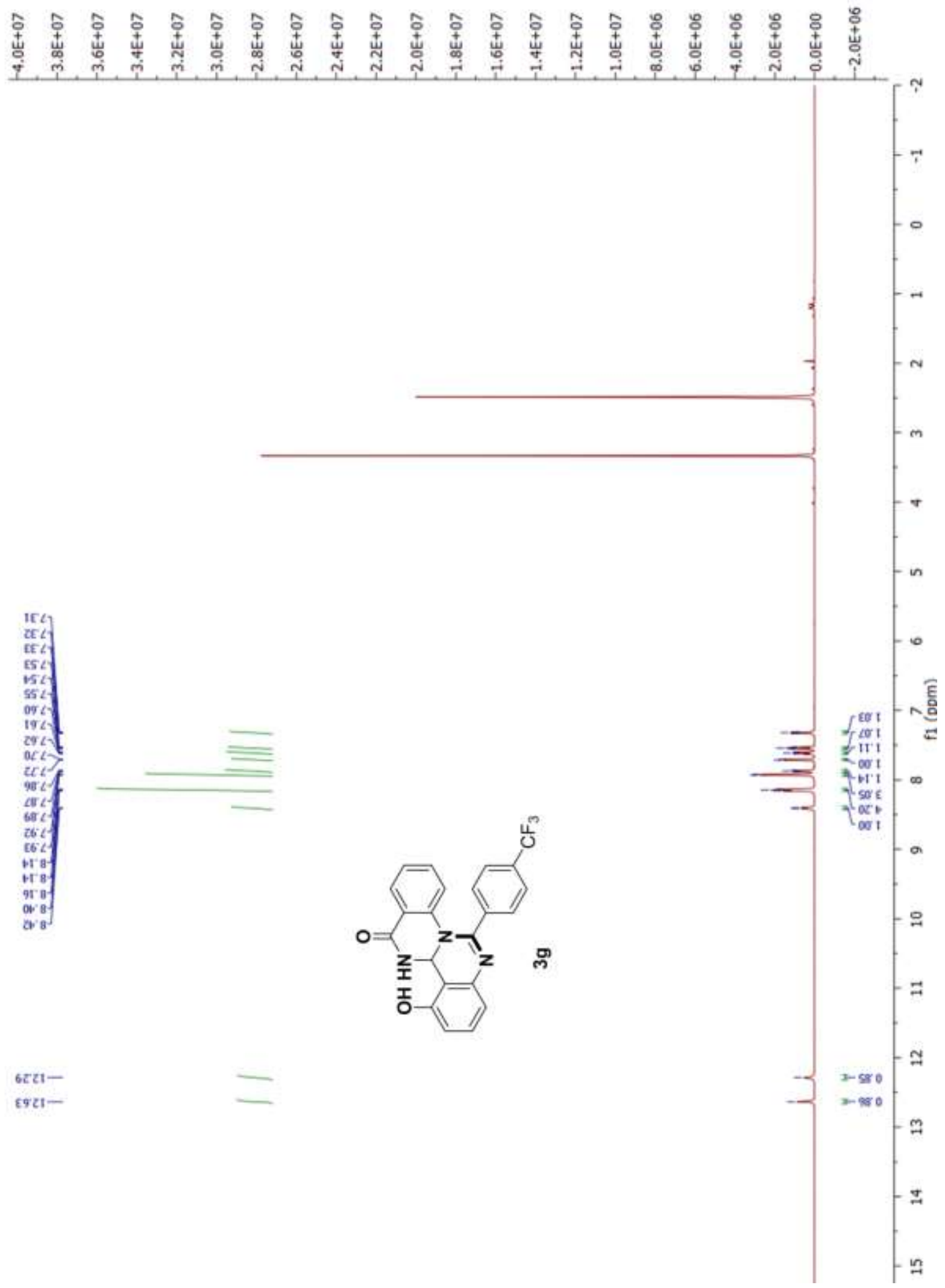


${ }^{13} \mathrm{C}$ NMR of Compound 3g:

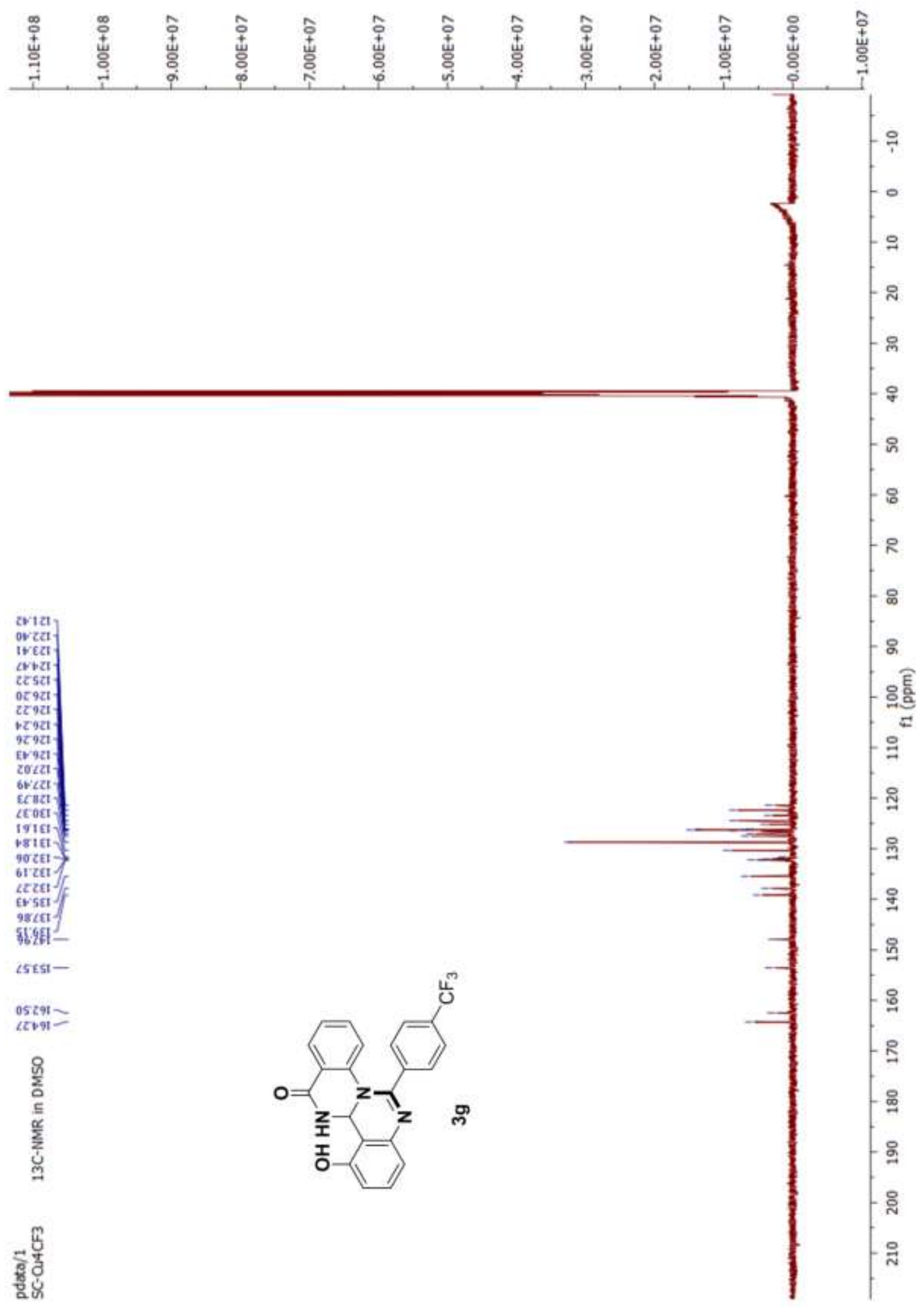


${ }^{1} \mathrm{H}$ NMR of Compound 3h:

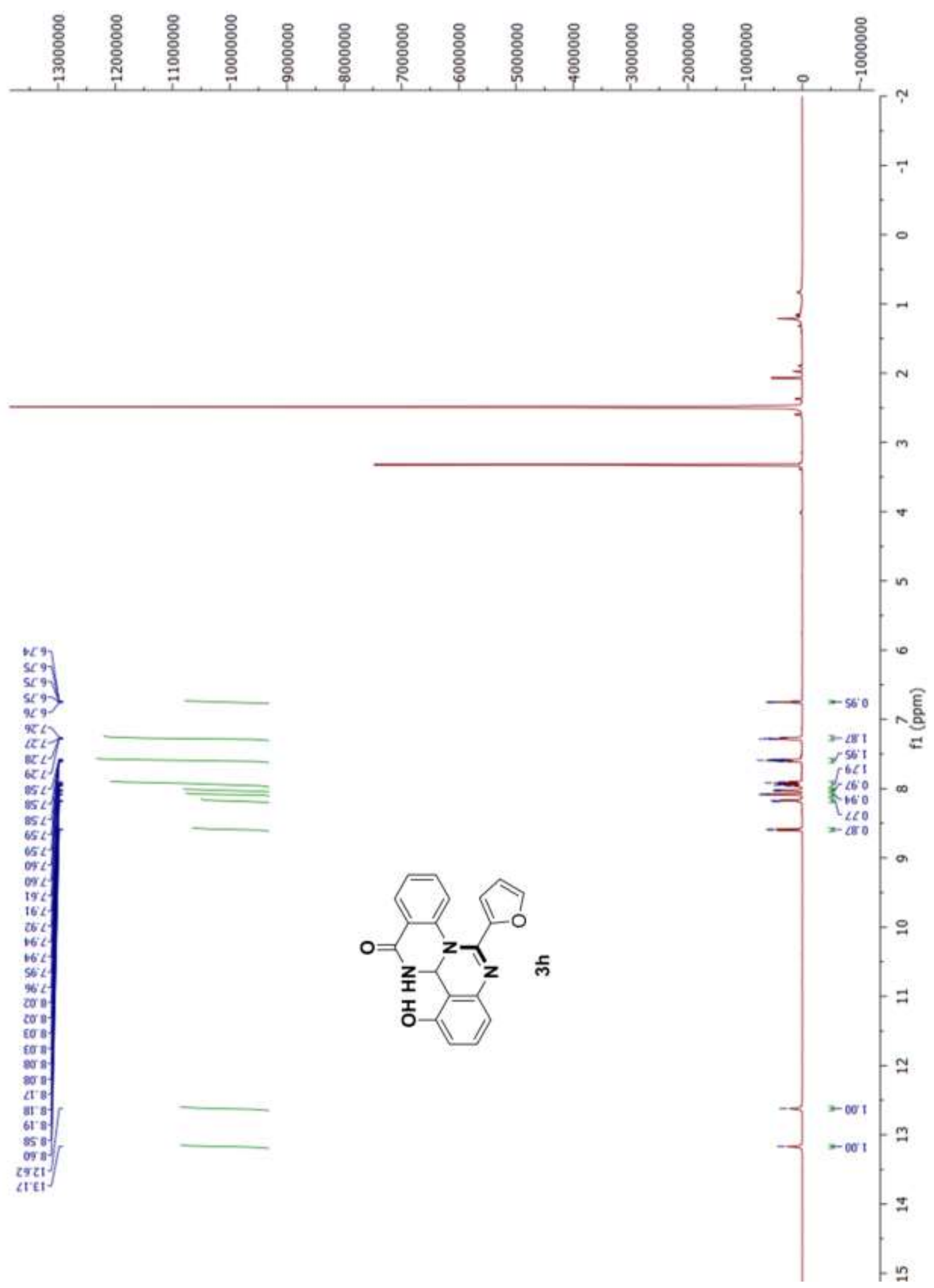


${ }^{13}$ C NMR of Compound 3h:

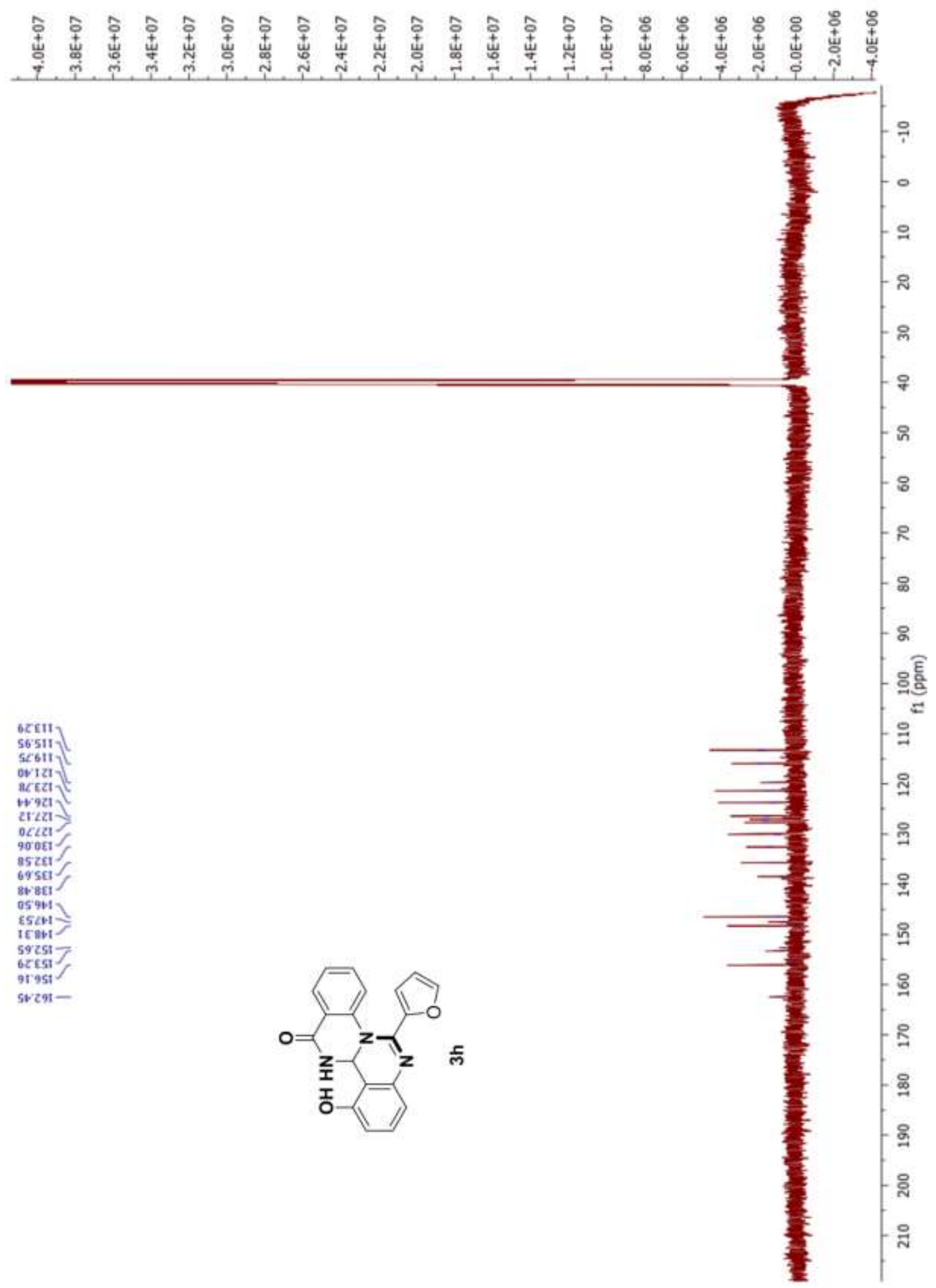


${ }^{1} \mathrm{H}$ NMR of Compound 3i:

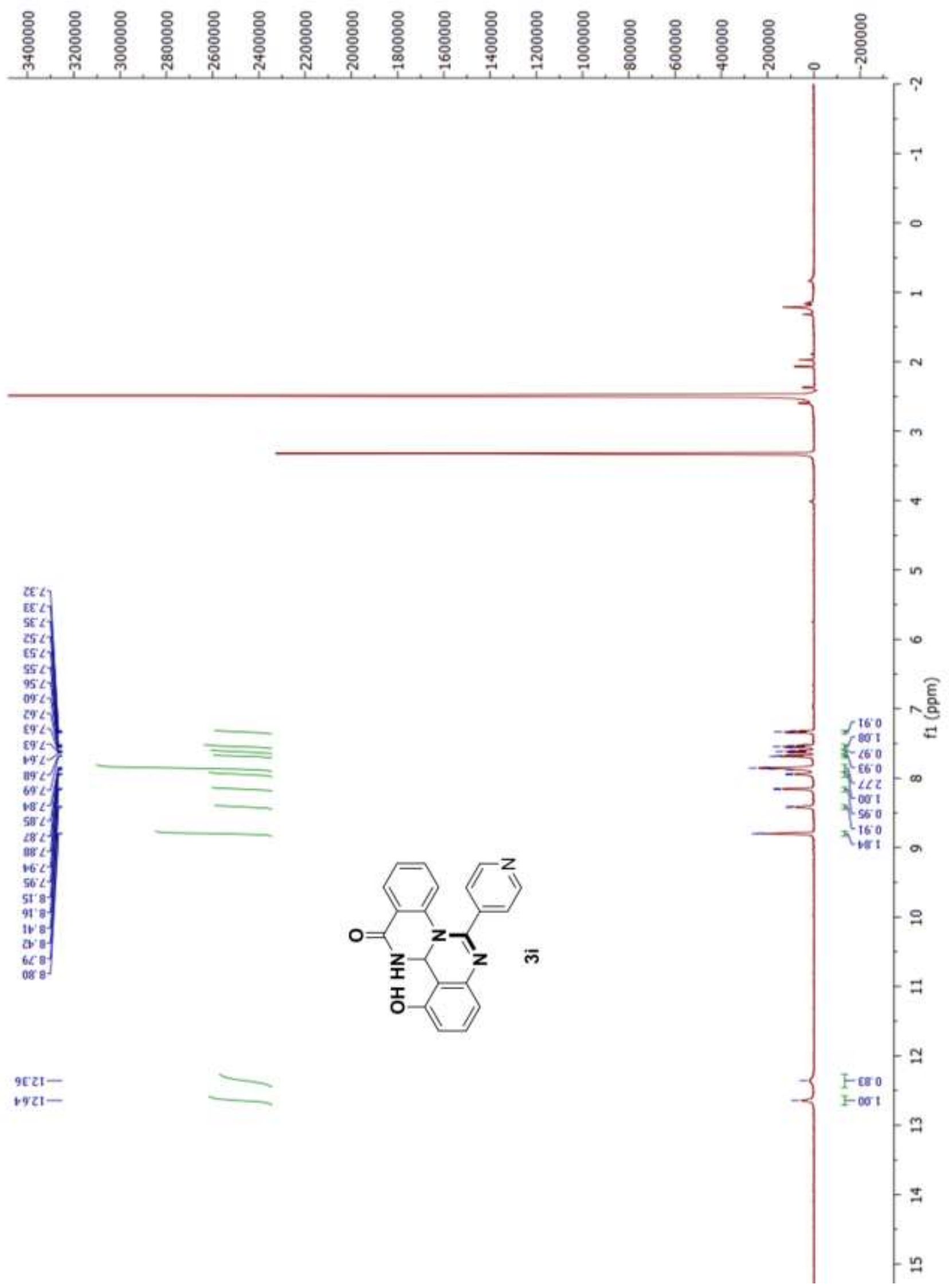


${ }^{13}$ C NMR of Compound 3i:

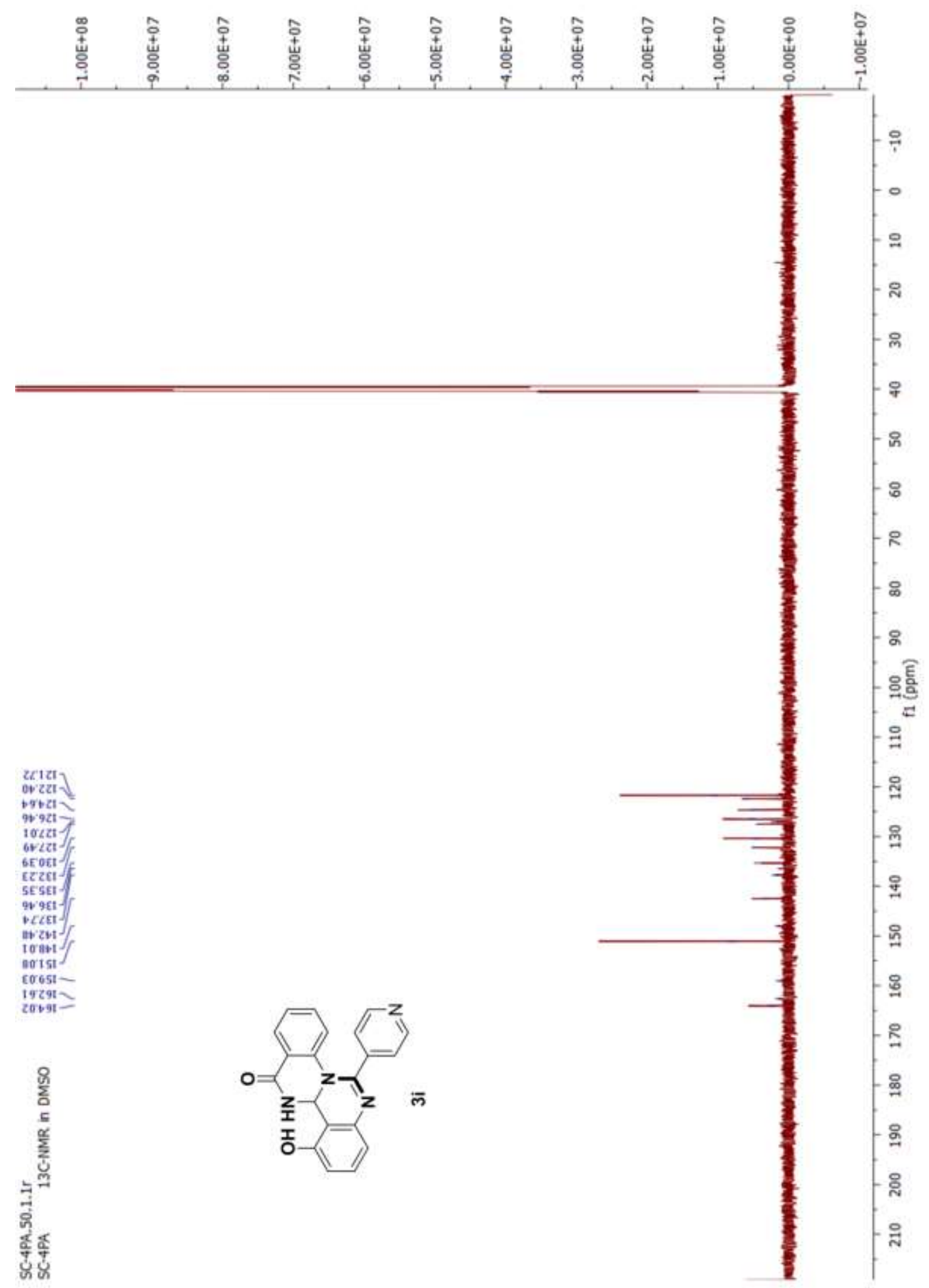


${ }^{1} \mathrm{H}$ NMR of Compound 4a:

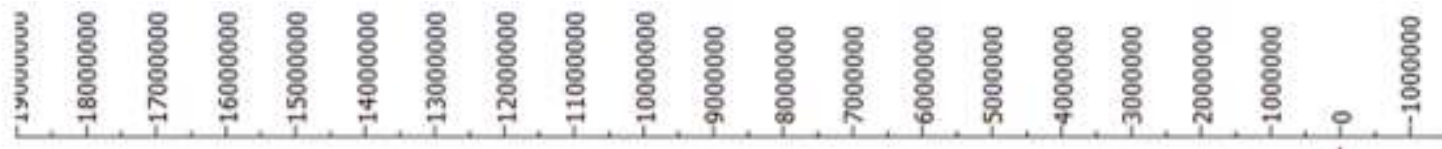

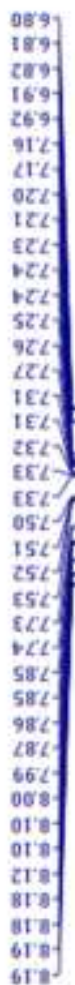

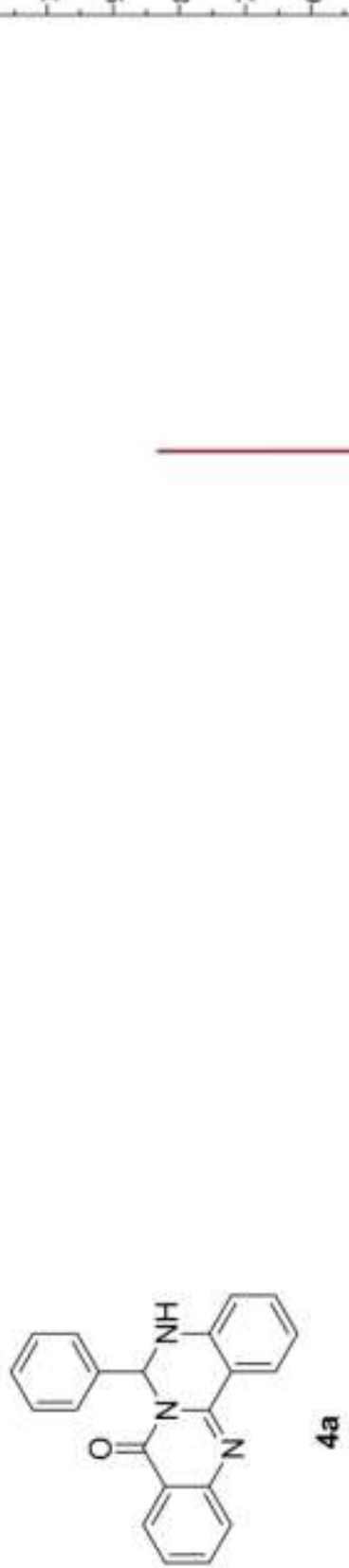


${ }^{13} \mathrm{C}$ NMR of Compound 4a:

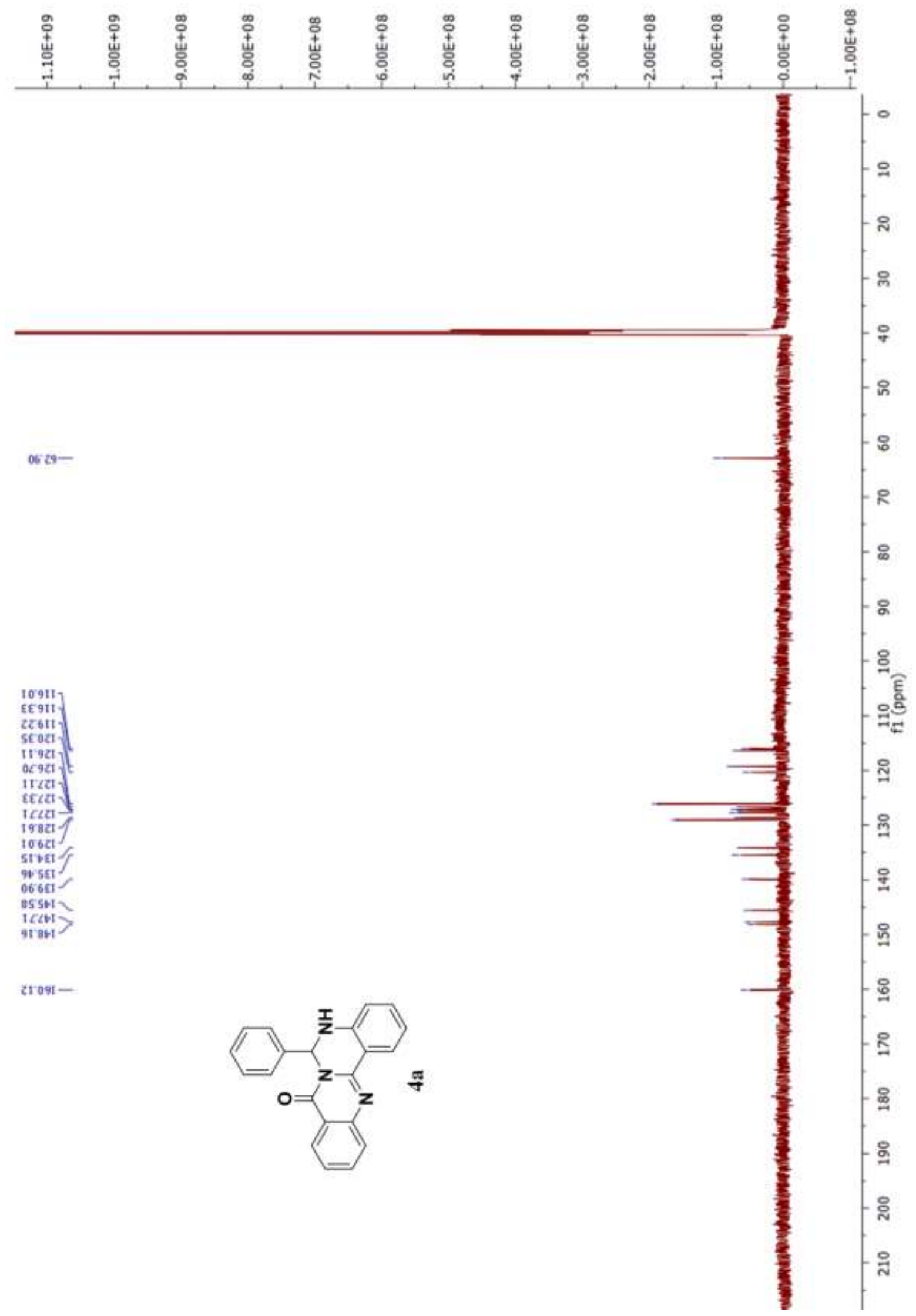


${ }^{1} \mathrm{H}$ NMR of Compound 4b:

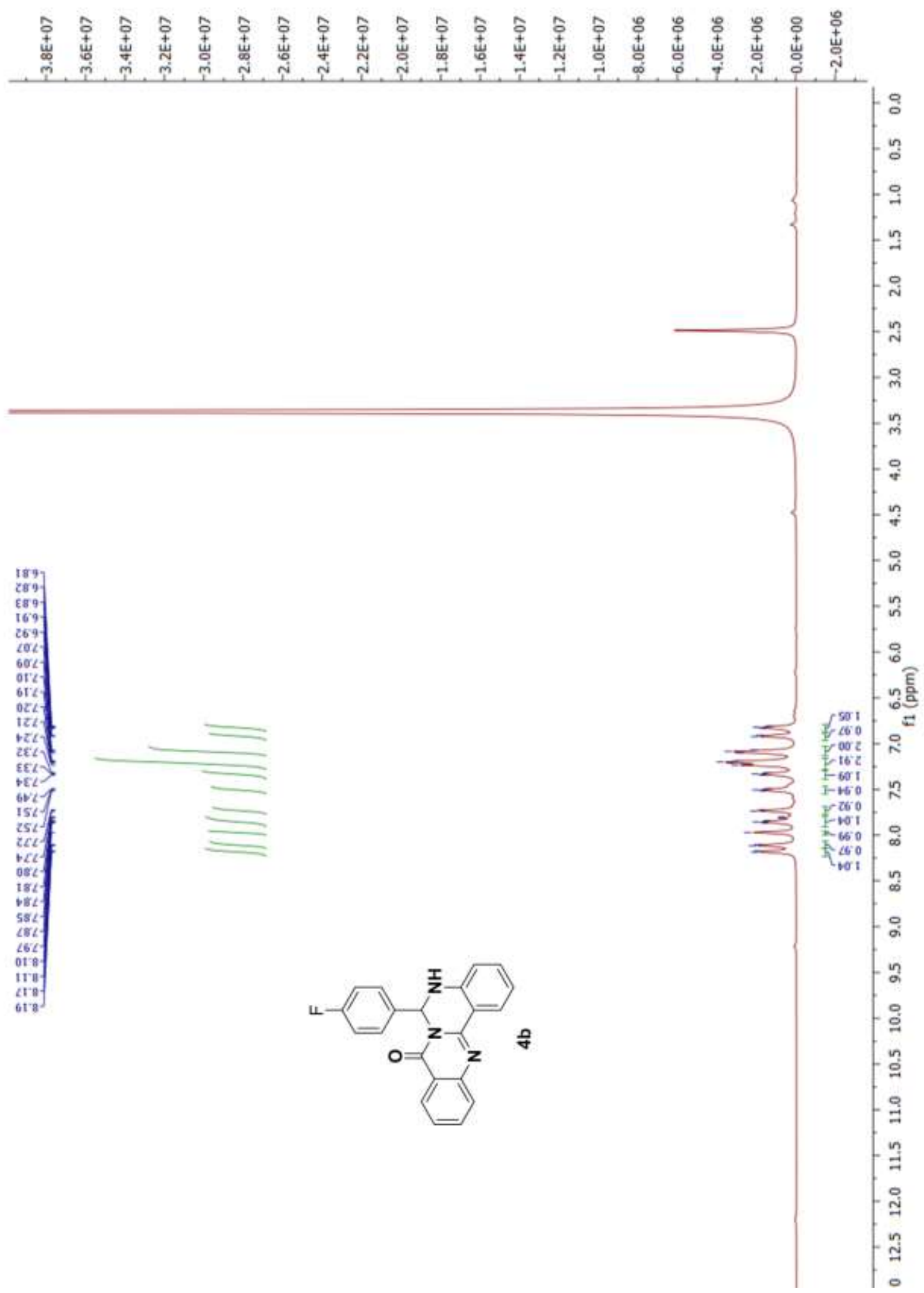


${ }^{13} \mathrm{C}$ NMR of Compound 4b:

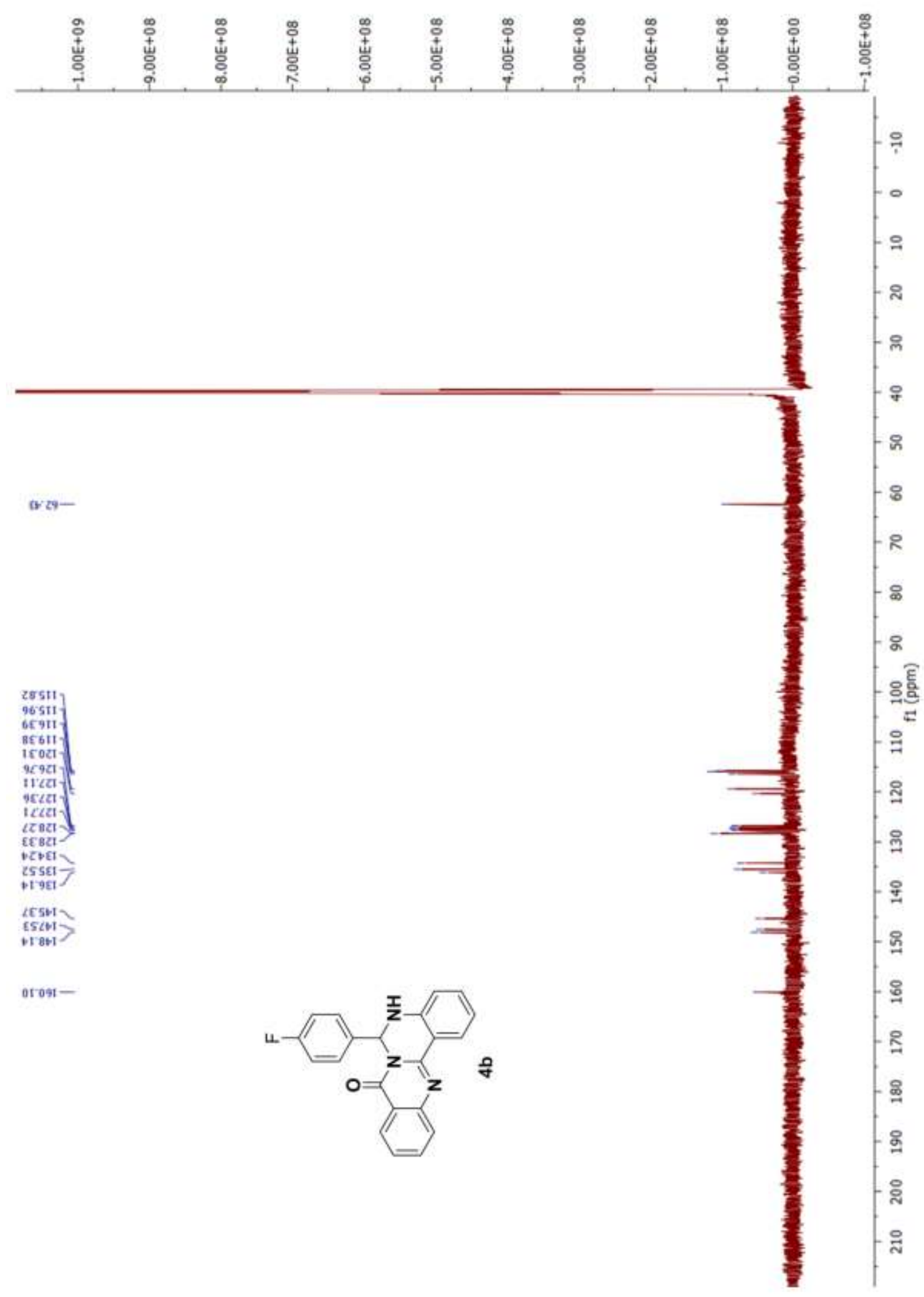


${ }^{1} \mathrm{H}$ NMR of Compound 4c:
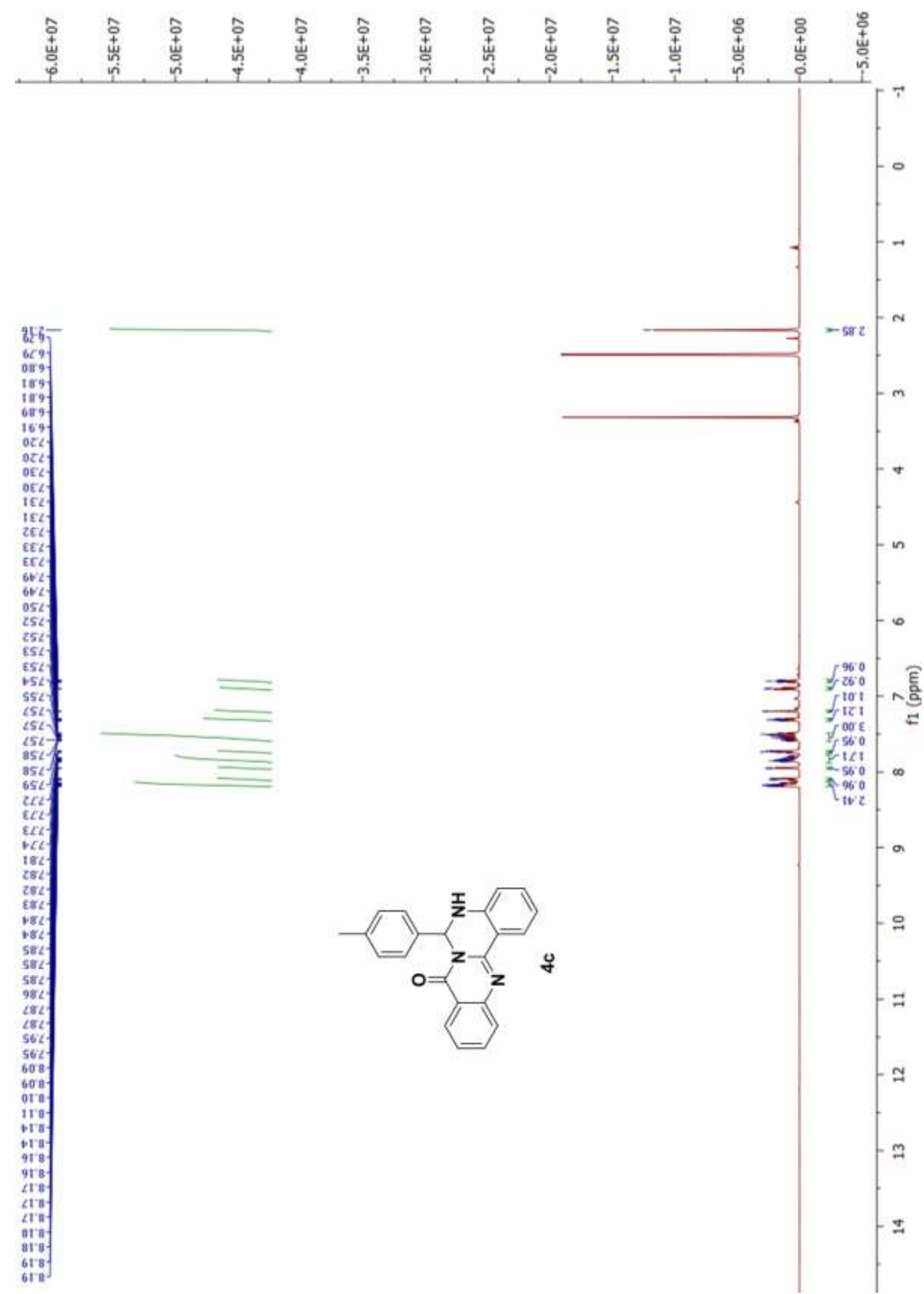
${ }^{13} \mathrm{C}$ NMR of Compound 4c:

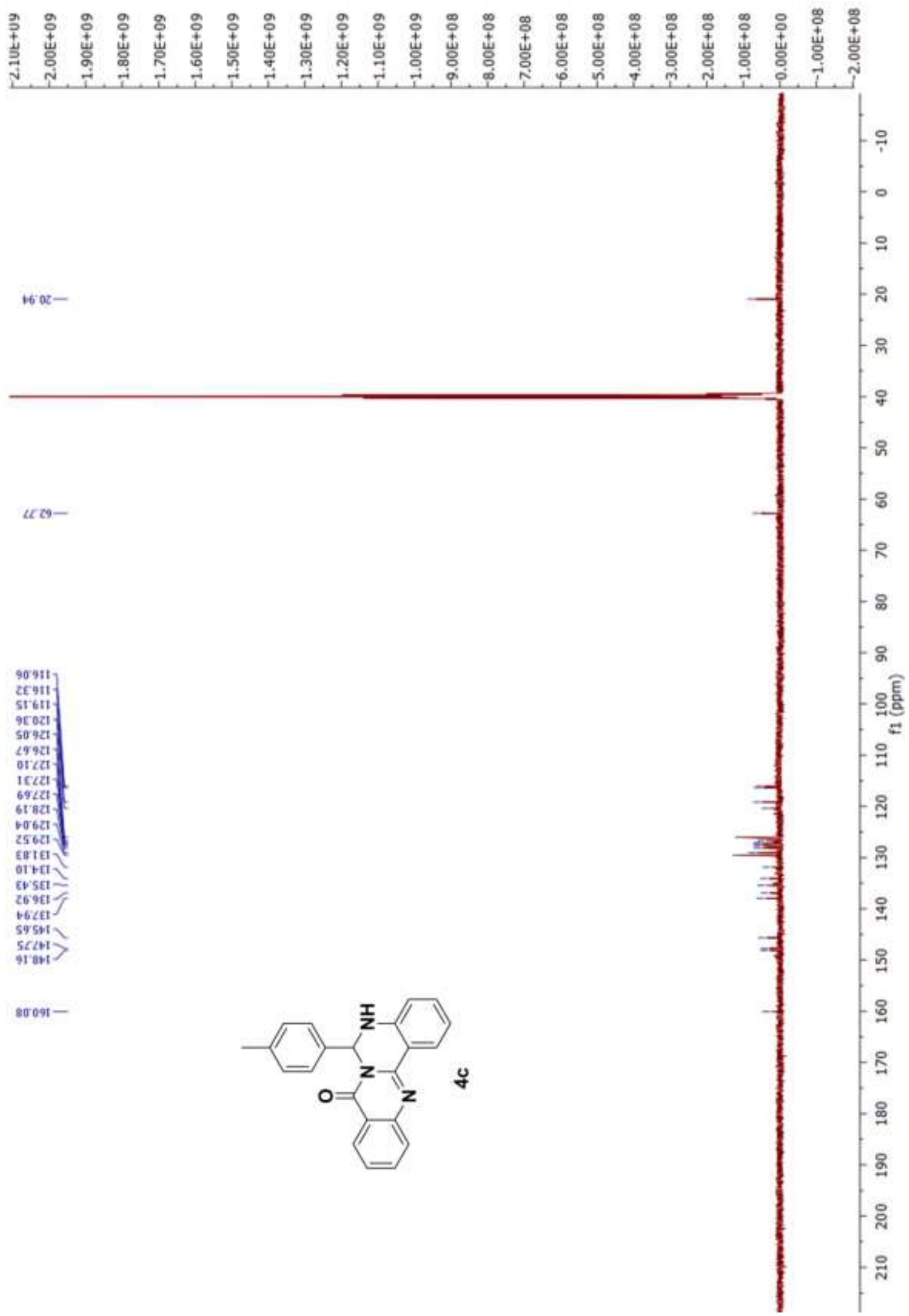


1H NMR of Compound 4d:
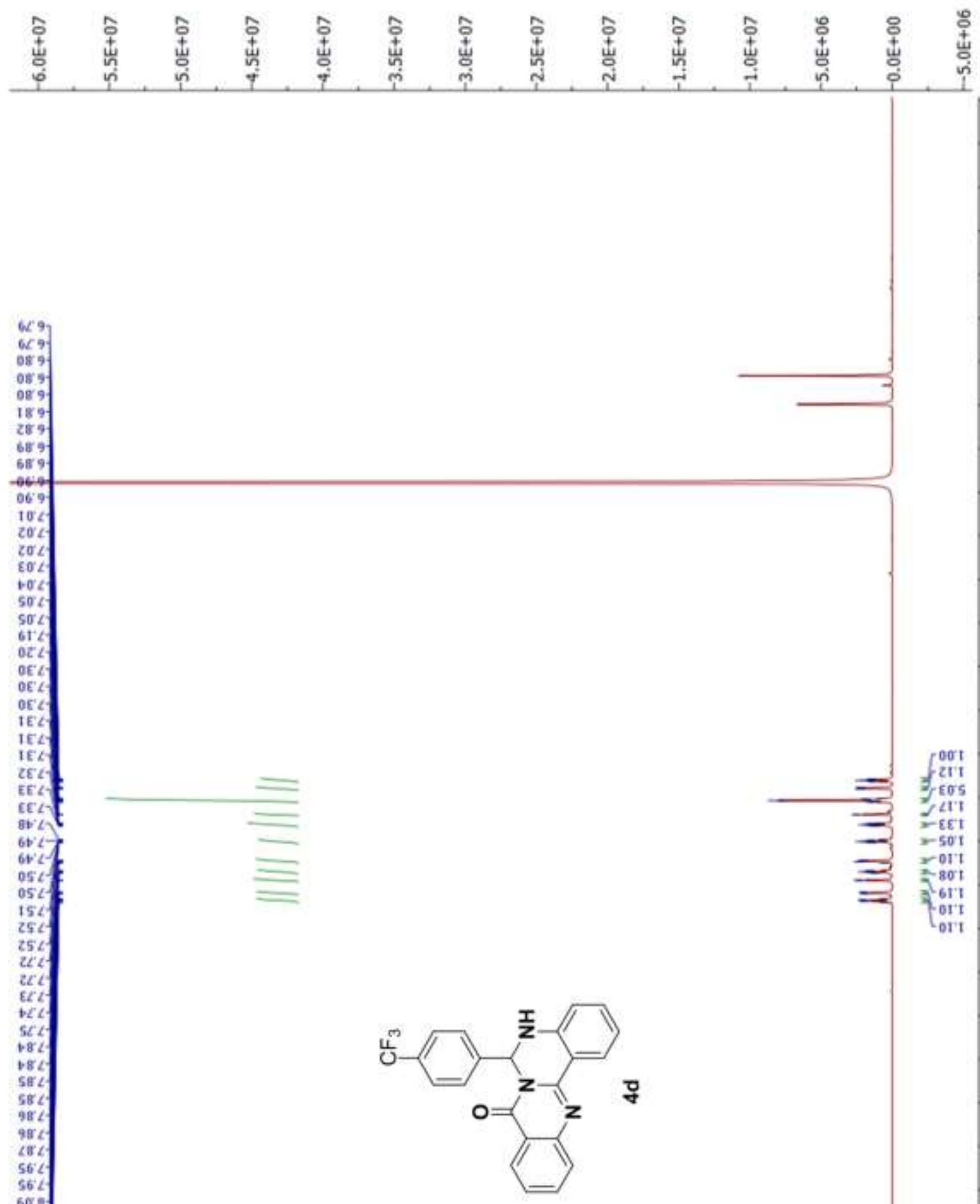

국

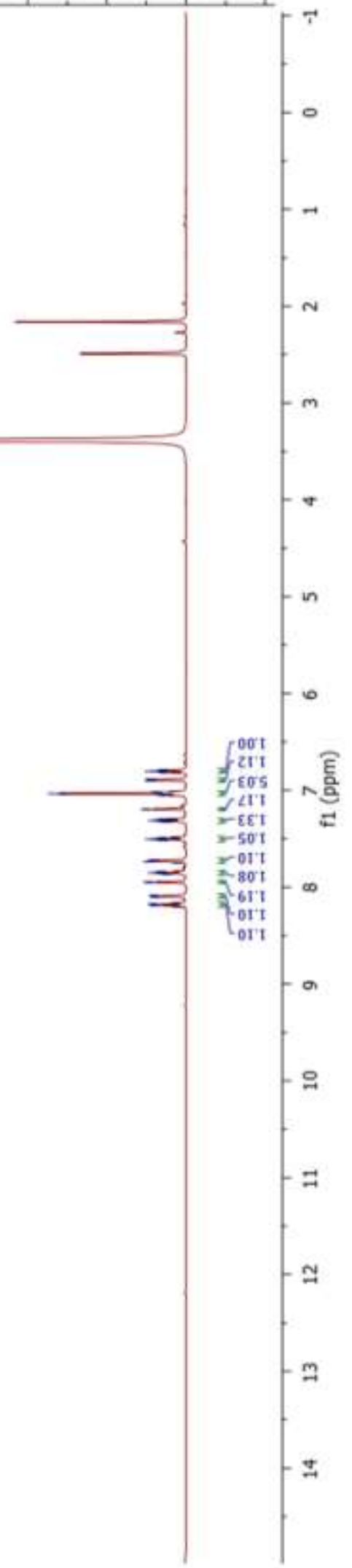


${ }^{13}$ C NMR of Compound 4d:

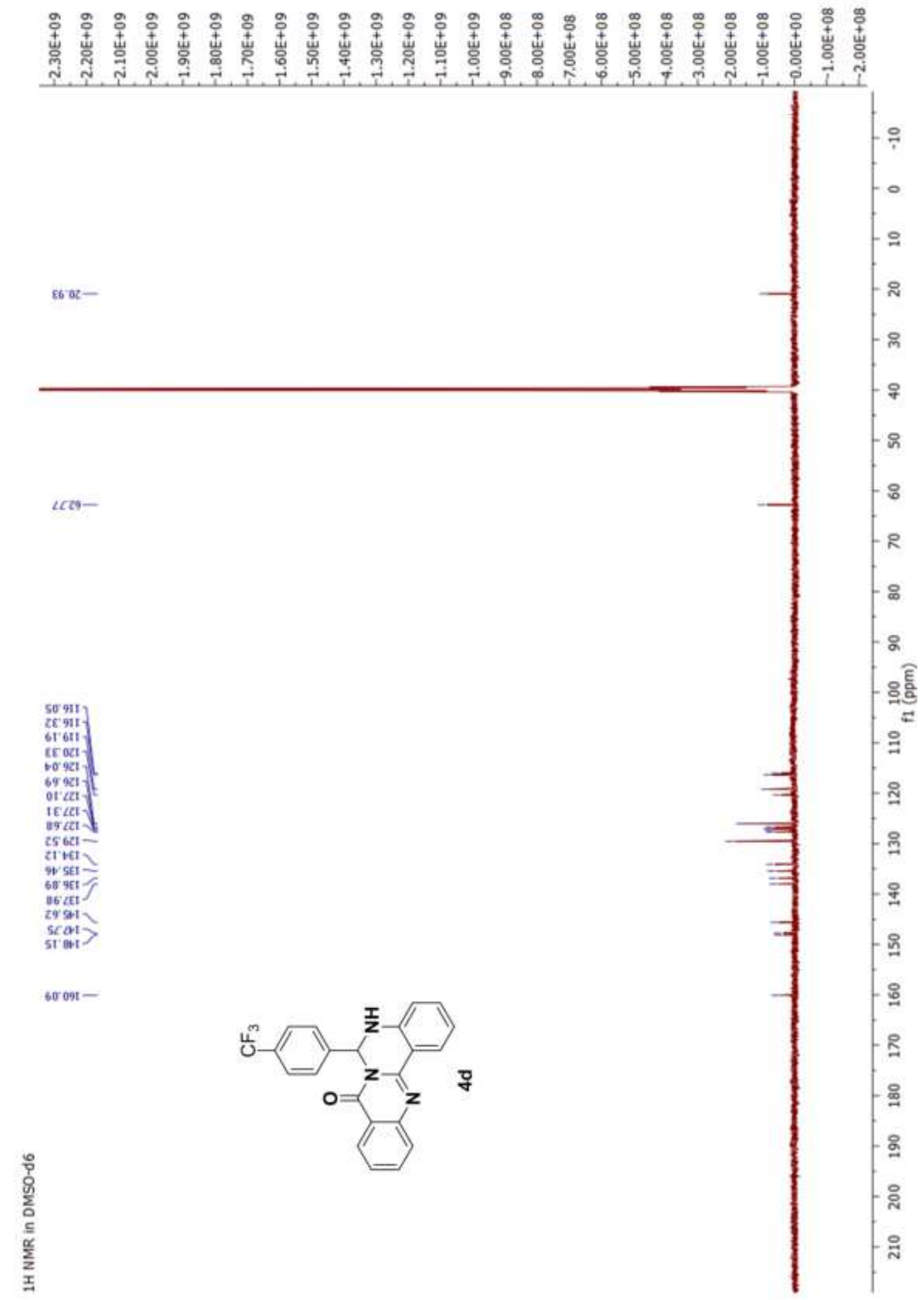


${ }^{1} \mathrm{H}$ NMR of Compound 4e:

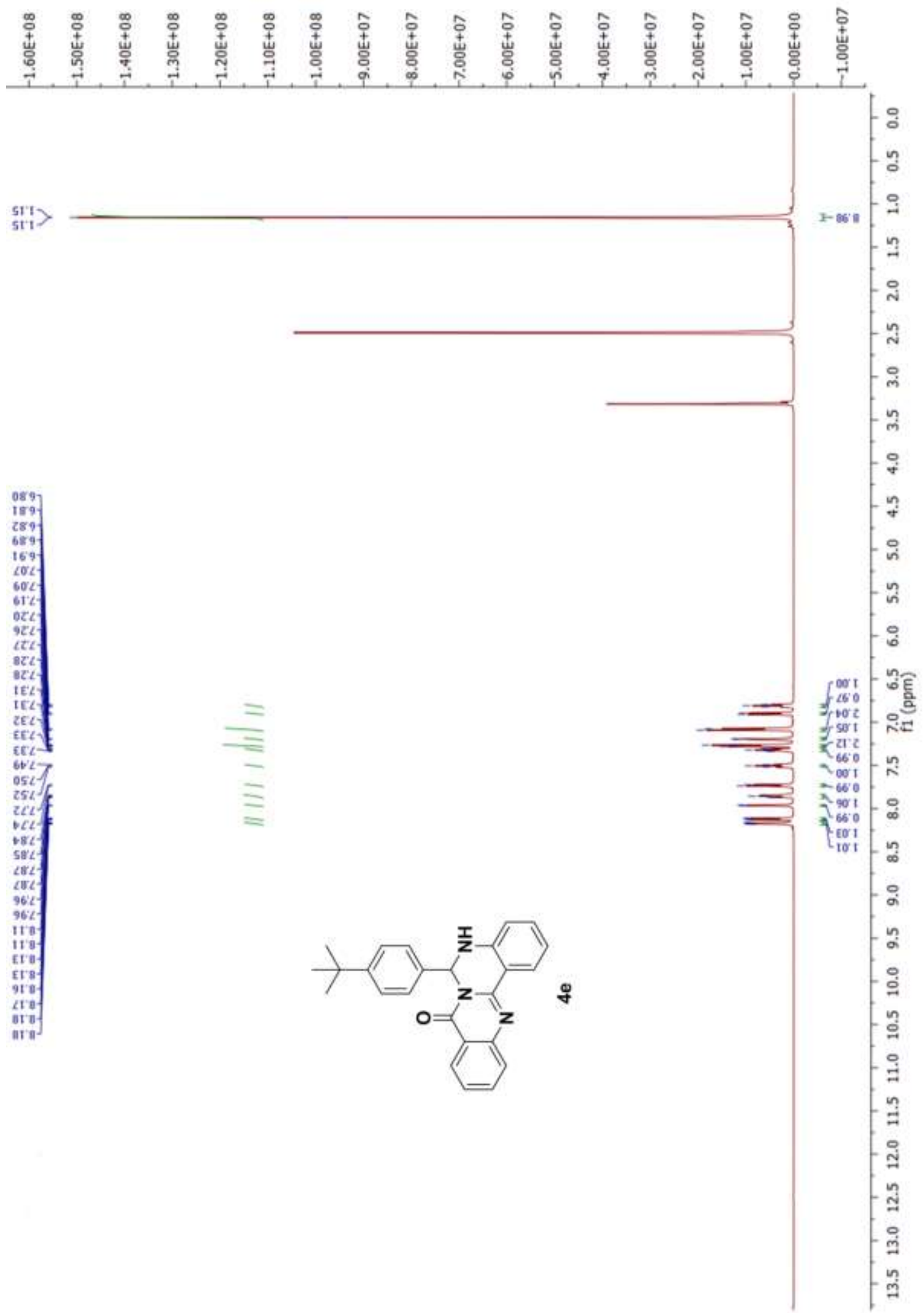


${ }^{13} \mathrm{C}$ NMR of Compound 4e:

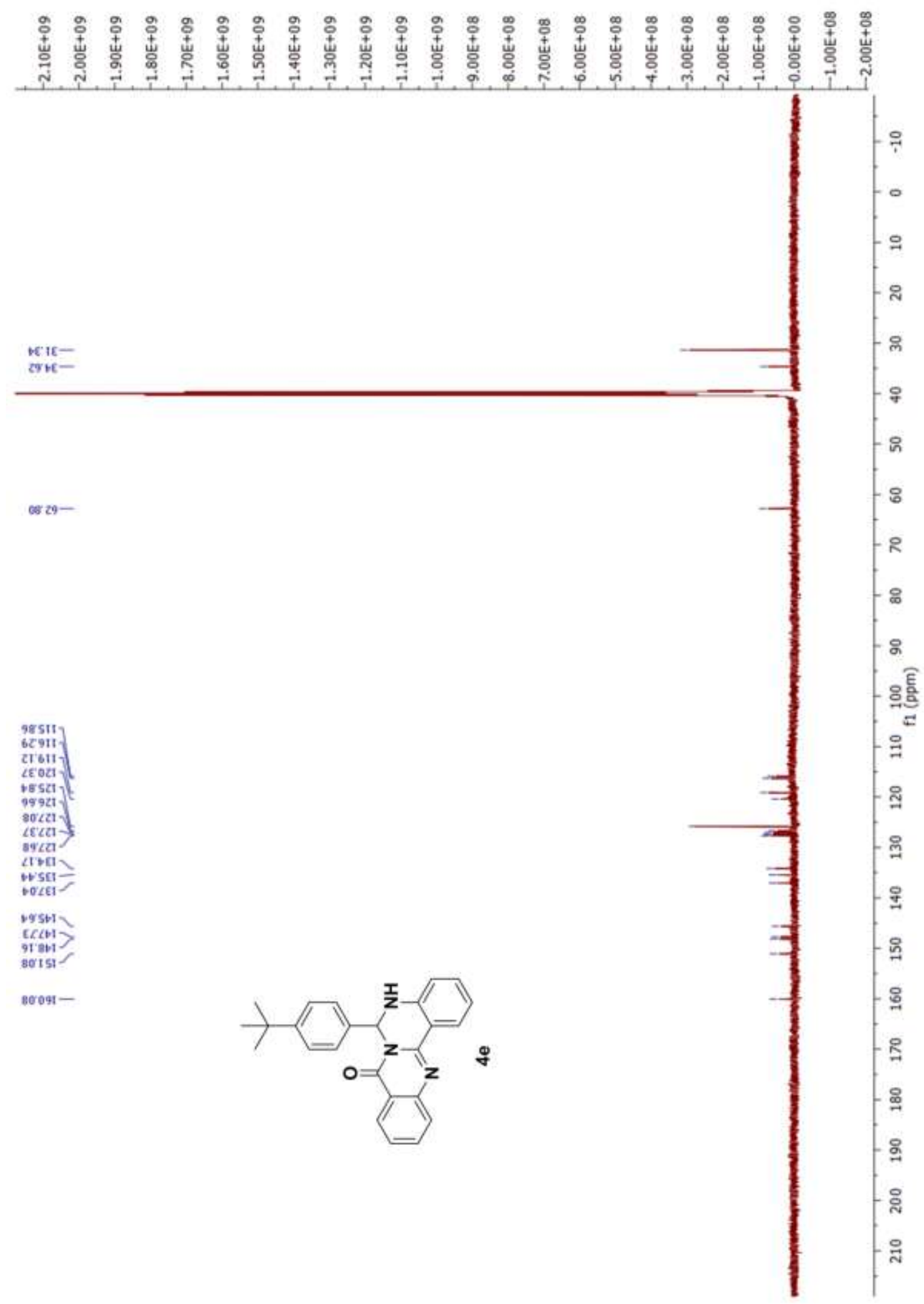


${ }^{1} \mathrm{H}$ NMR of Compound 4f:
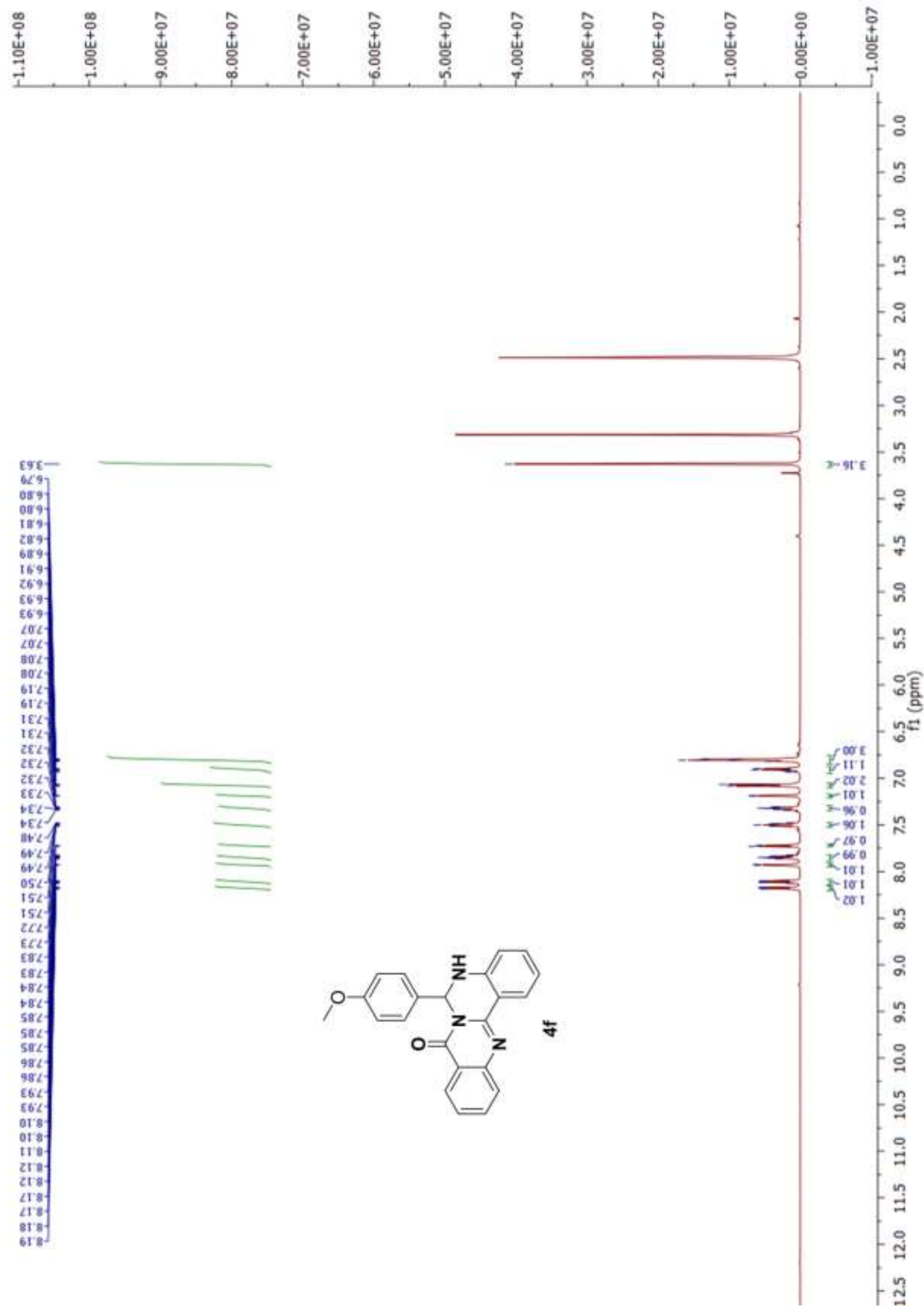
${ }^{13}$ C NMR of Compound 4f:

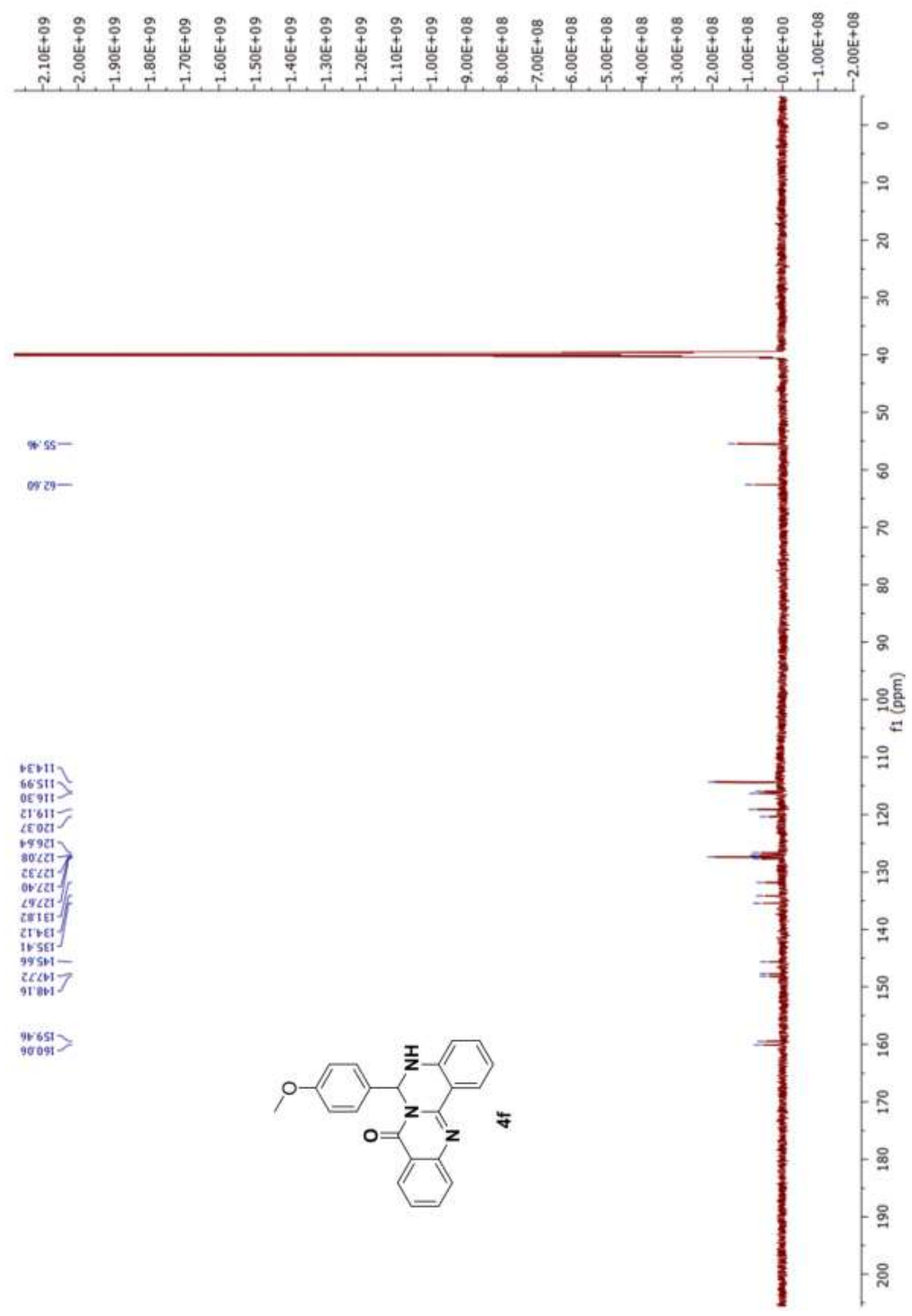


${ }^{1} \mathrm{H}$ NMR of Compound 4f':

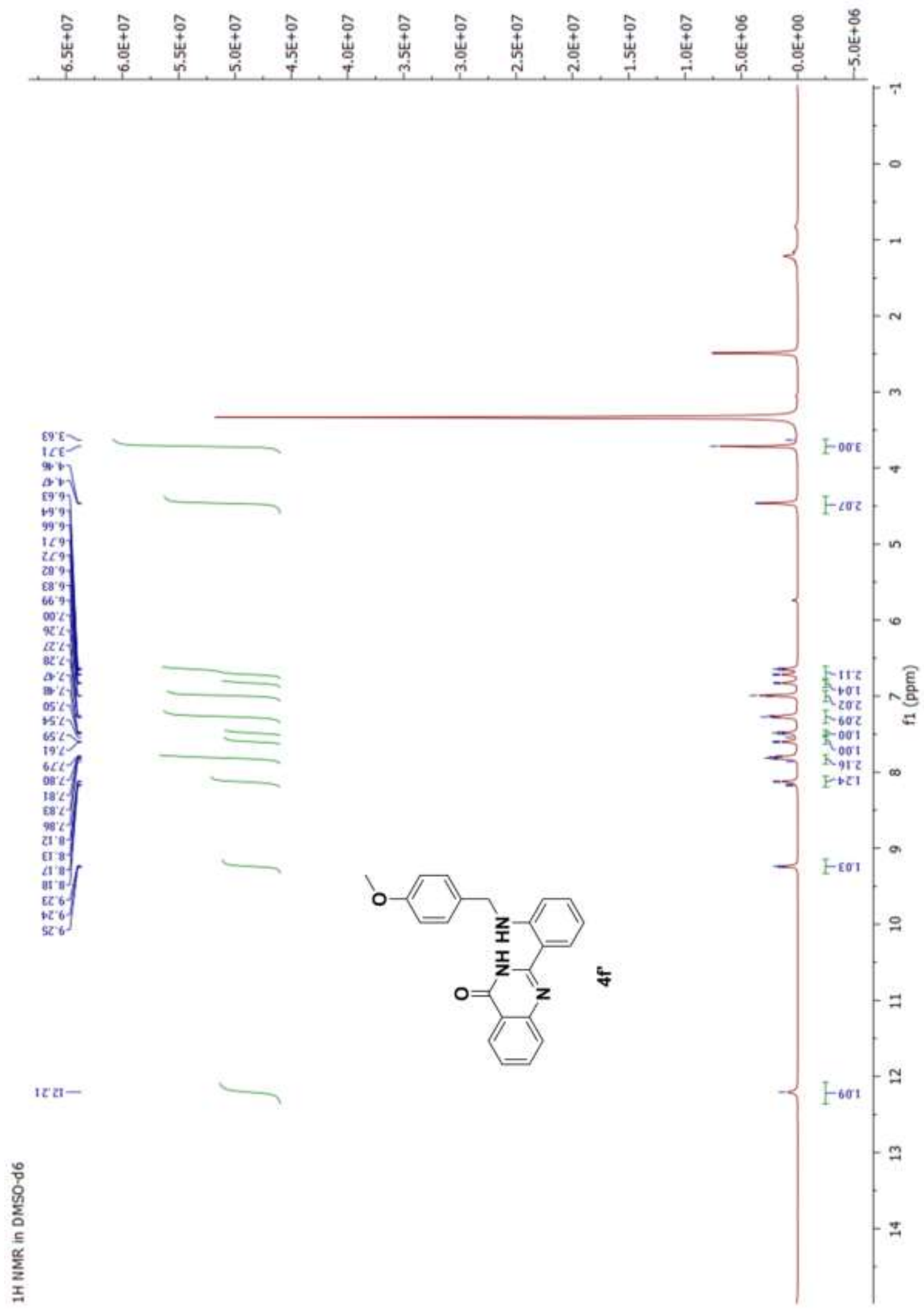


${ }^{13}$ C NMR of Compound 4f':

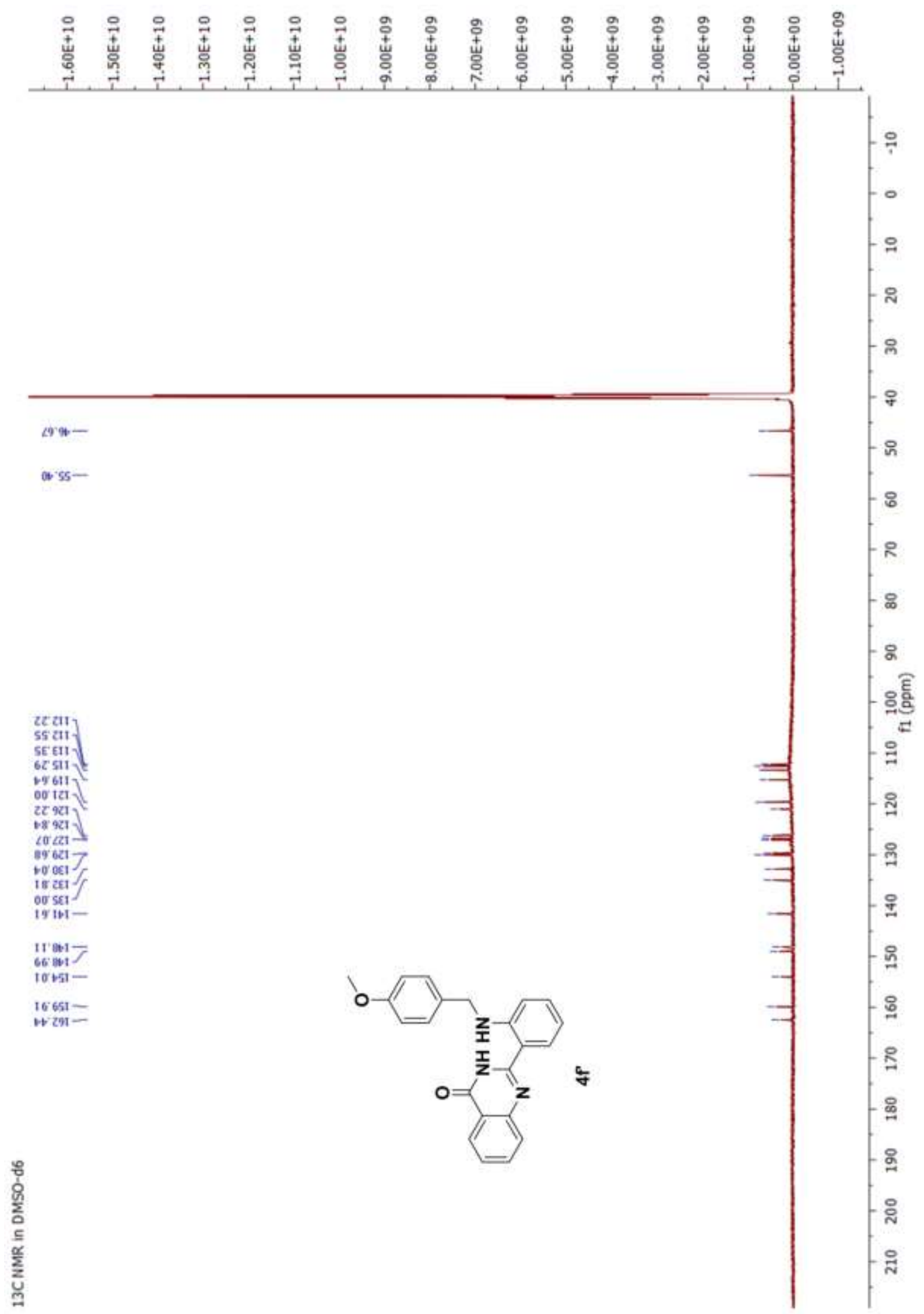


${ }^{1} \mathrm{H}$ NMR of Compound 4g:

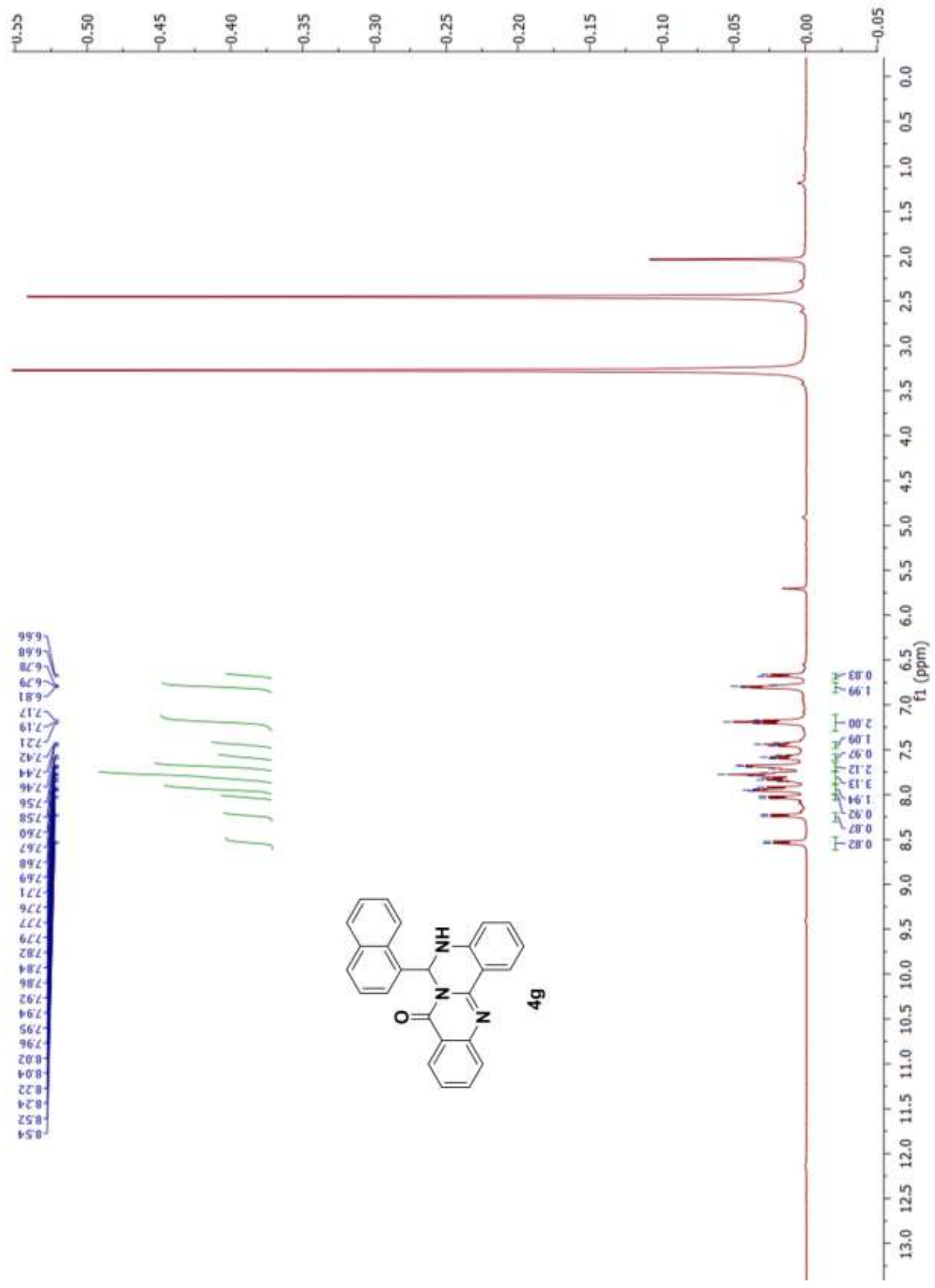


${ }^{13} \mathrm{C}$ NMR of Compound 4g:

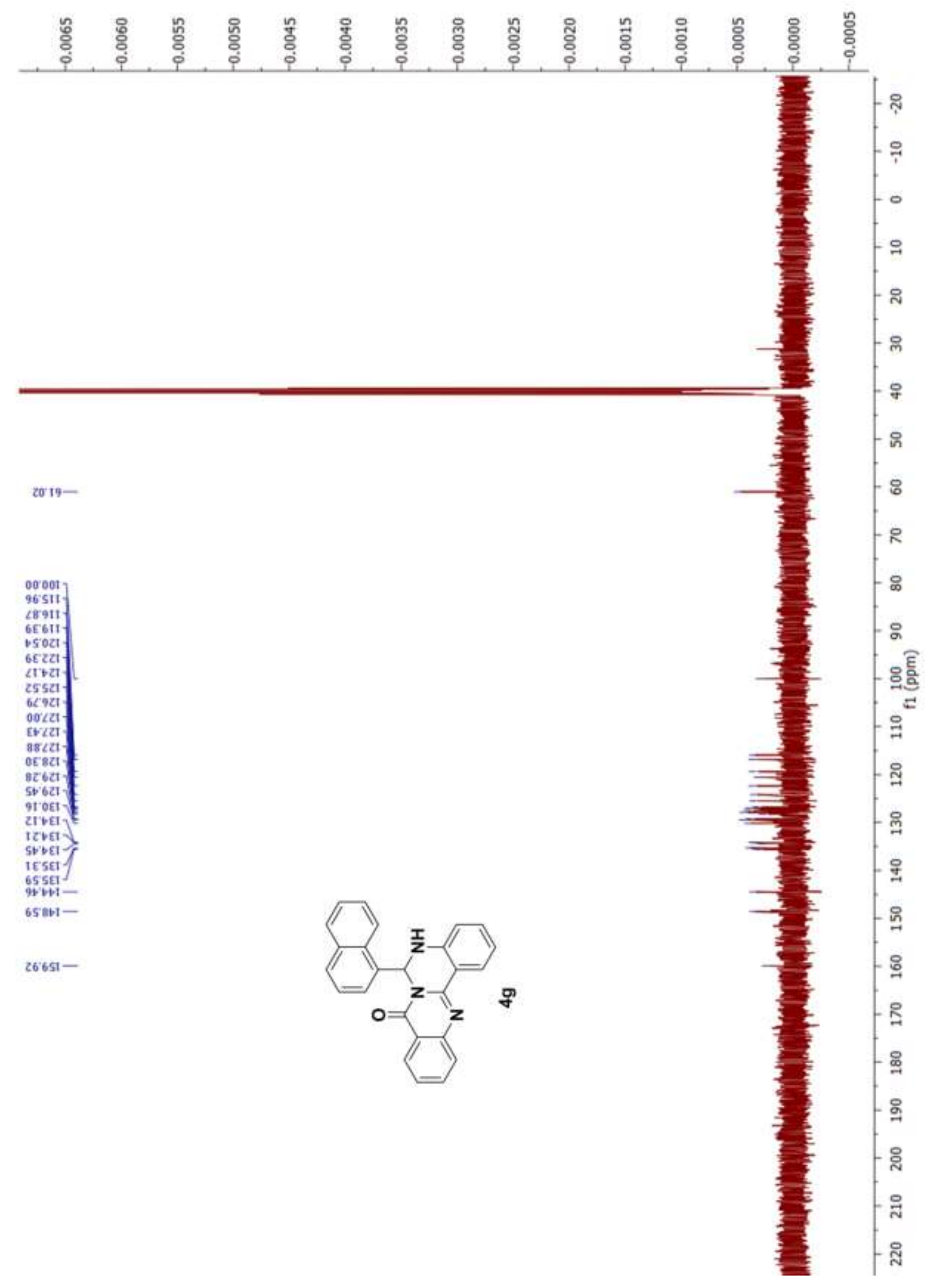


${ }^{1} \mathrm{H}$ NMR of Compound 4h:
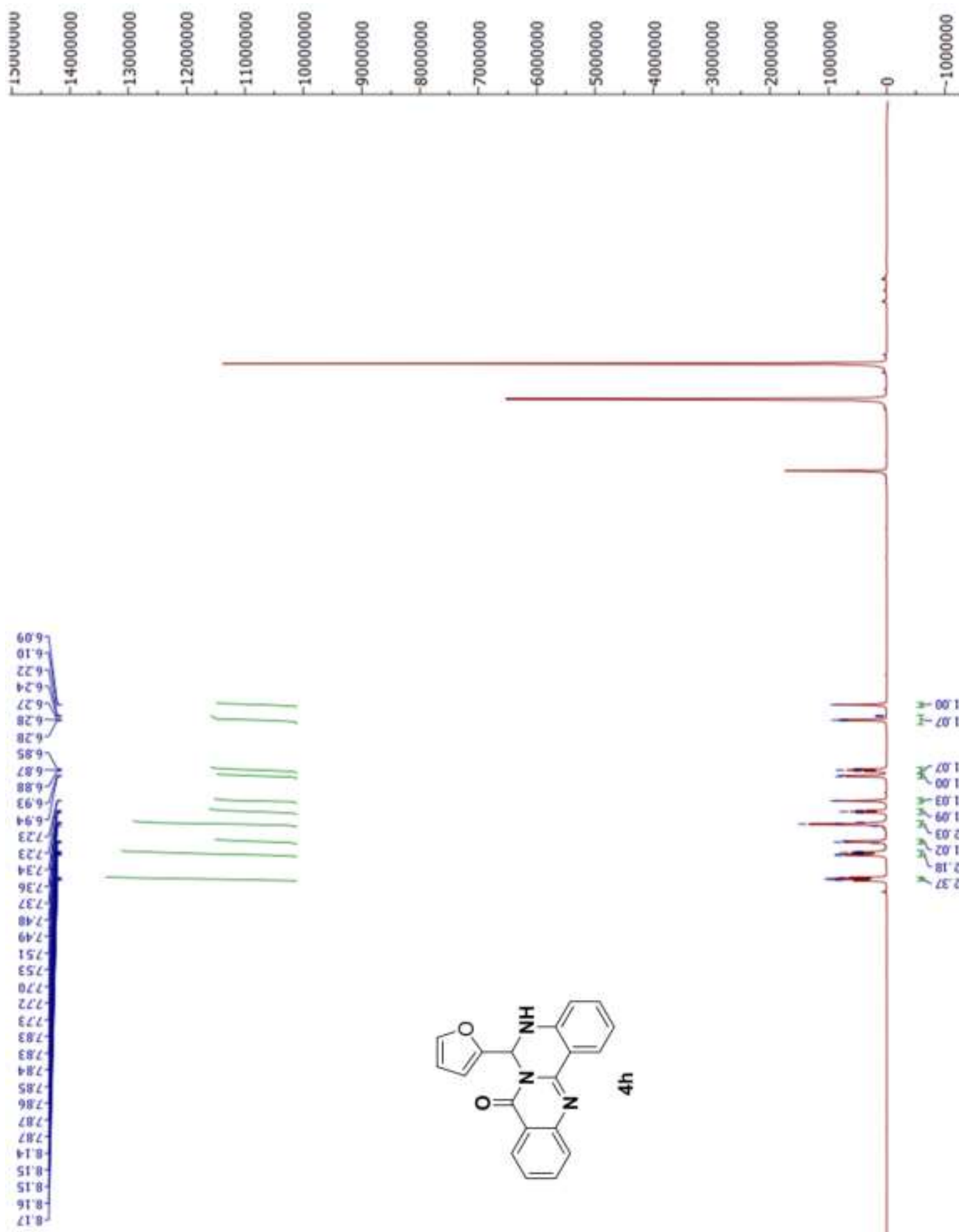

f 
${ }^{13} \mathrm{C}$ NMR of Compound 4h:

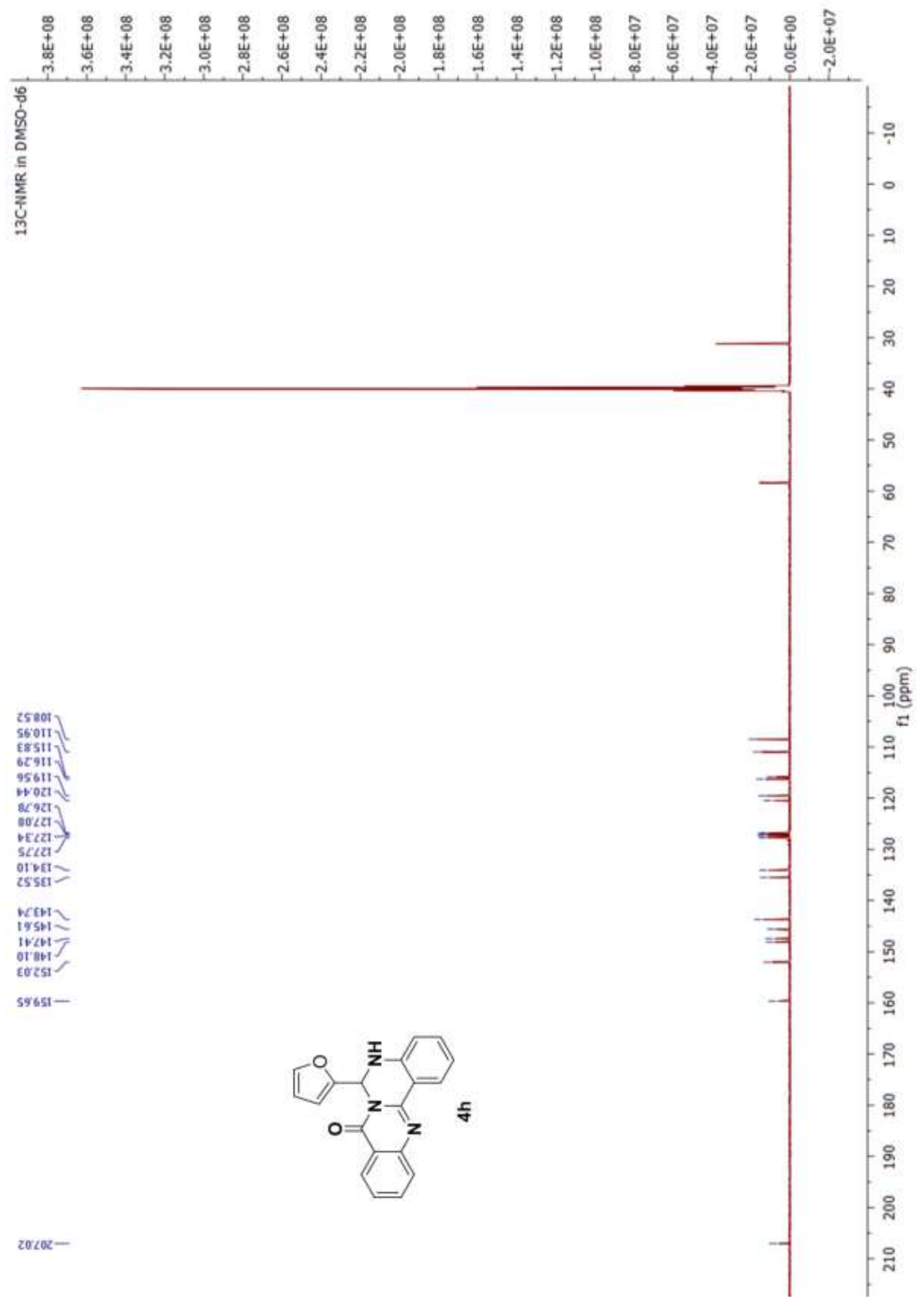


${ }^{1} \mathrm{H}$ NMR of Compound 4i:

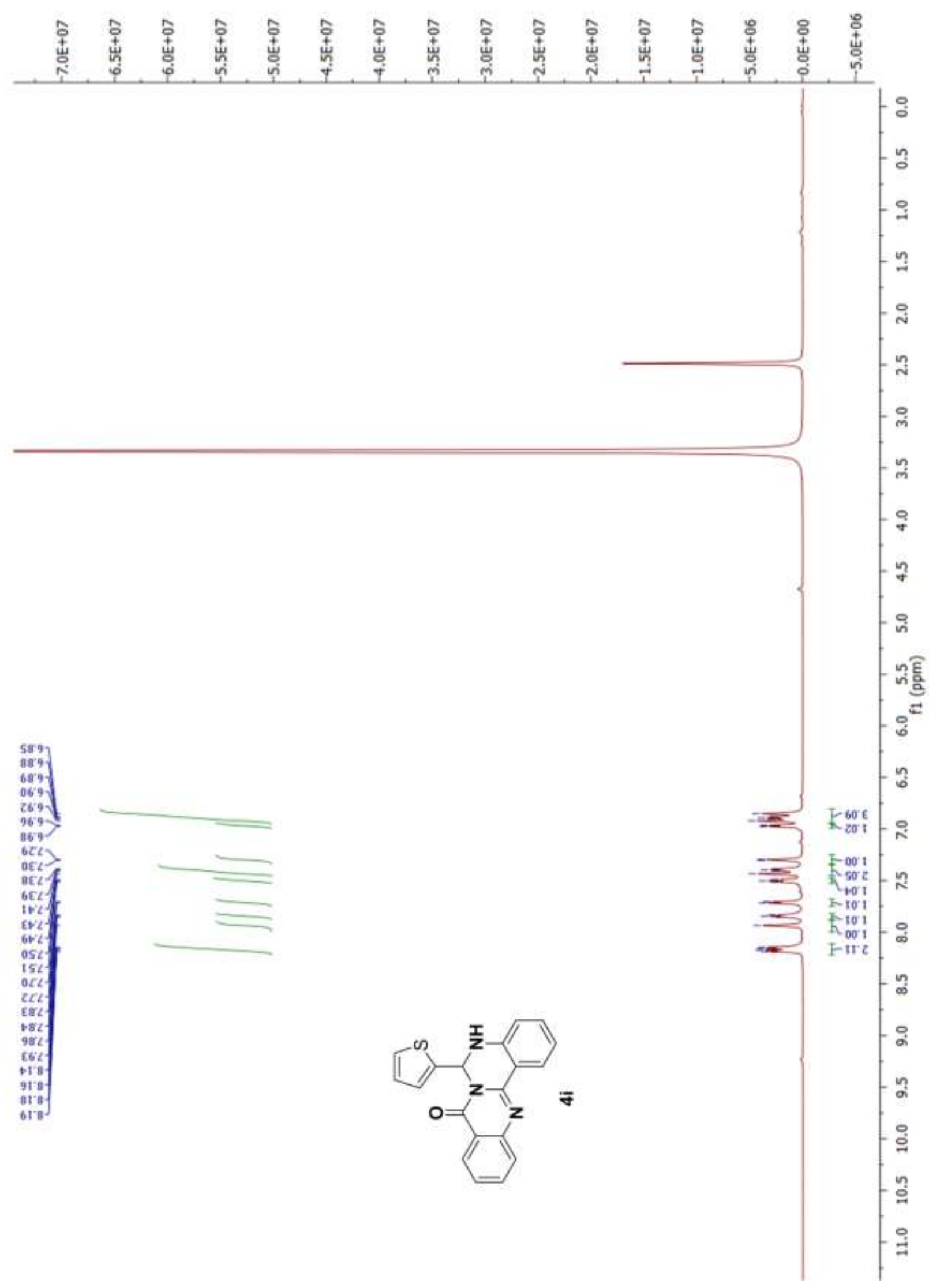


${ }^{13}$ C NMR of Compound 4i:

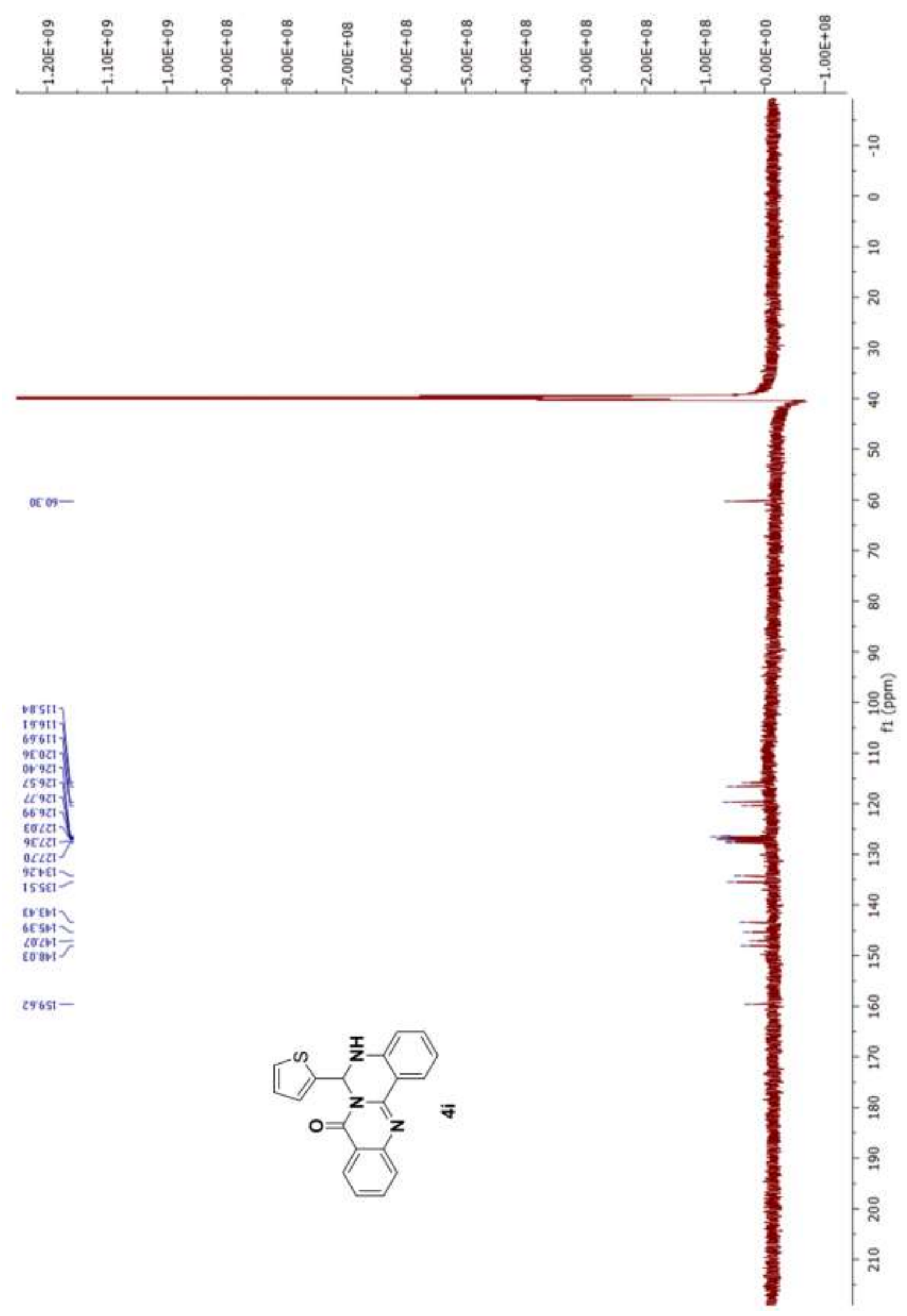


${ }^{1} \mathrm{H}$ NMR of Compound 4j:
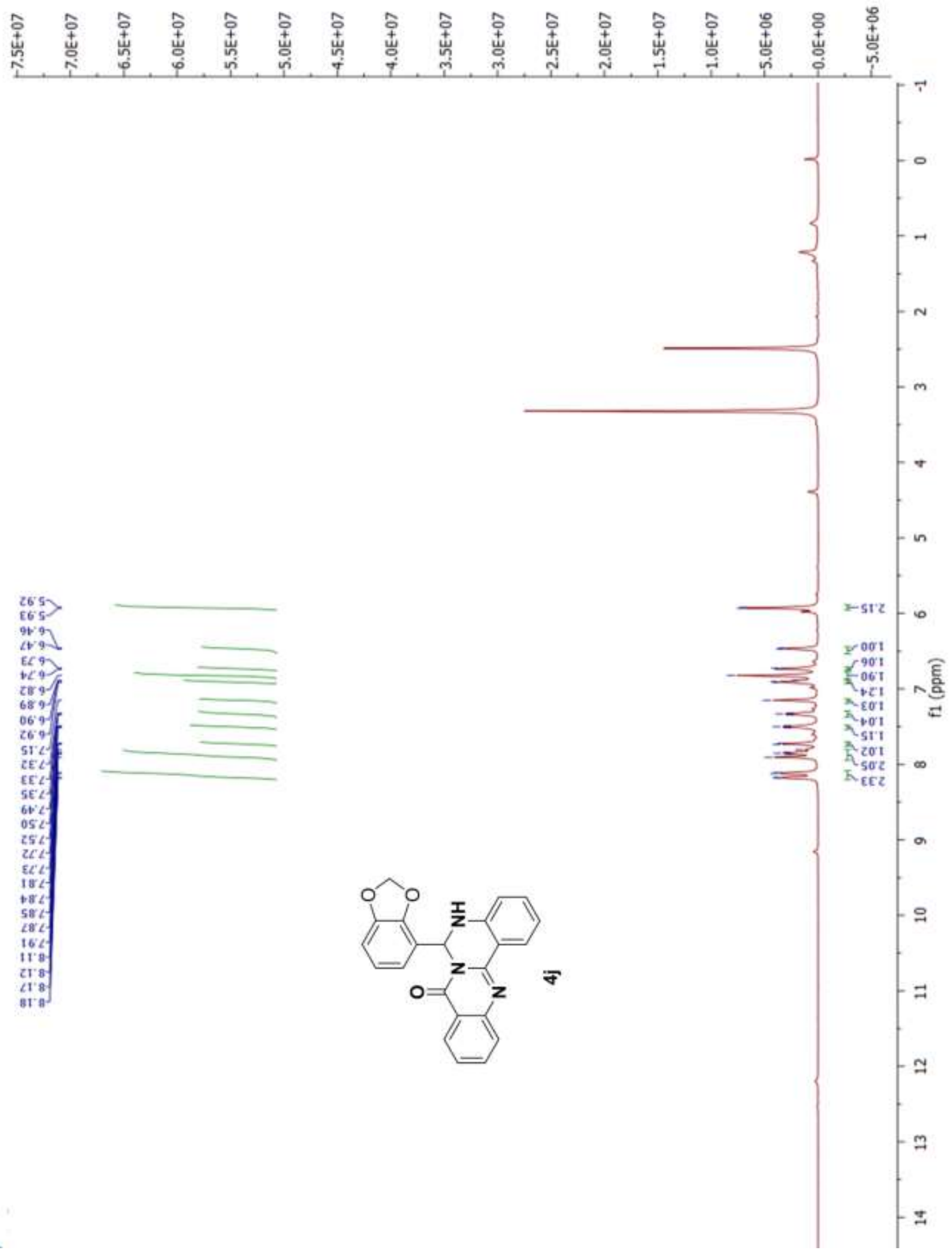
${ }^{13} \mathrm{C}$ NMR of Compound 4j:

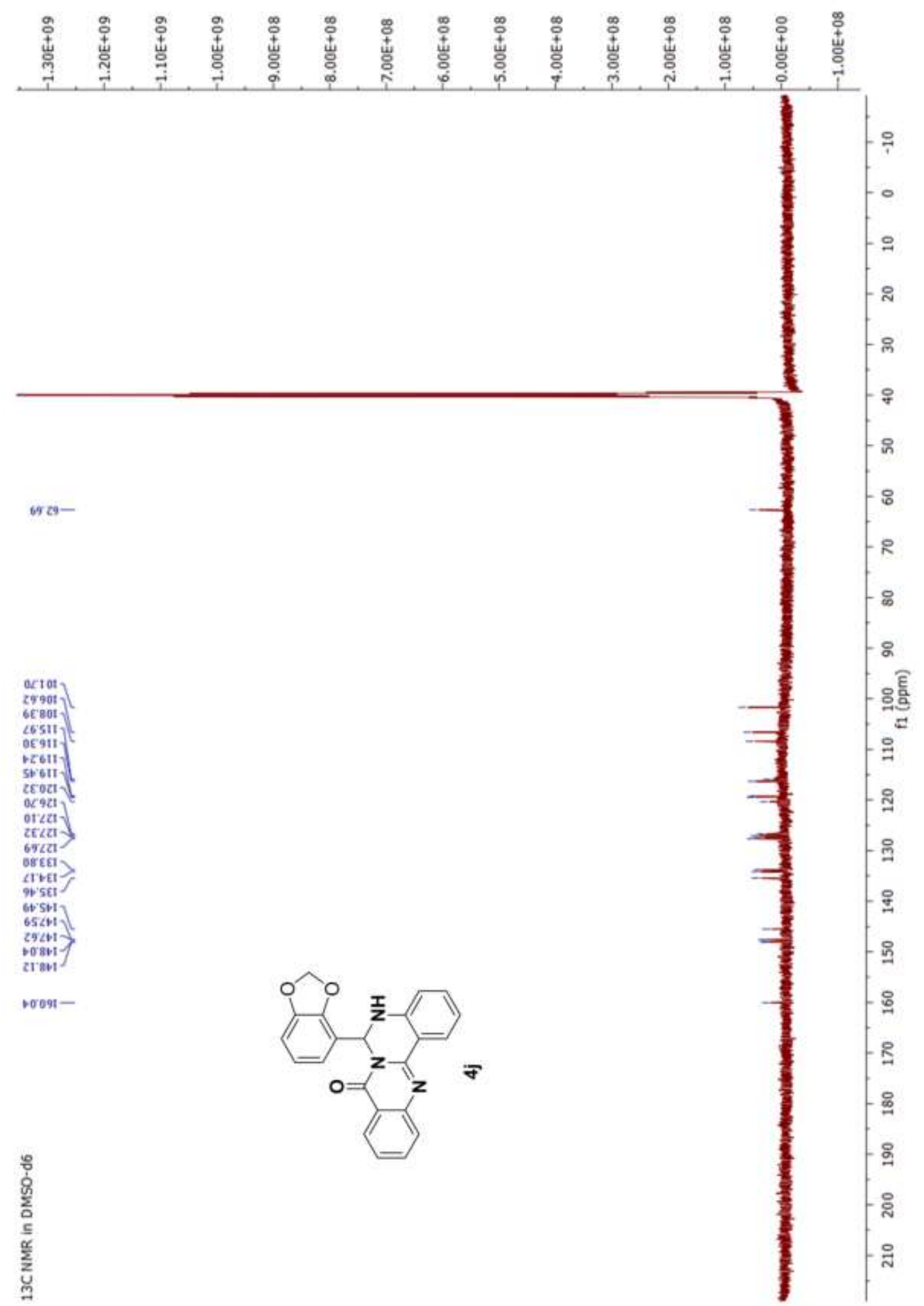


${ }^{1} \mathrm{H}$ NMR of Compound 4k:

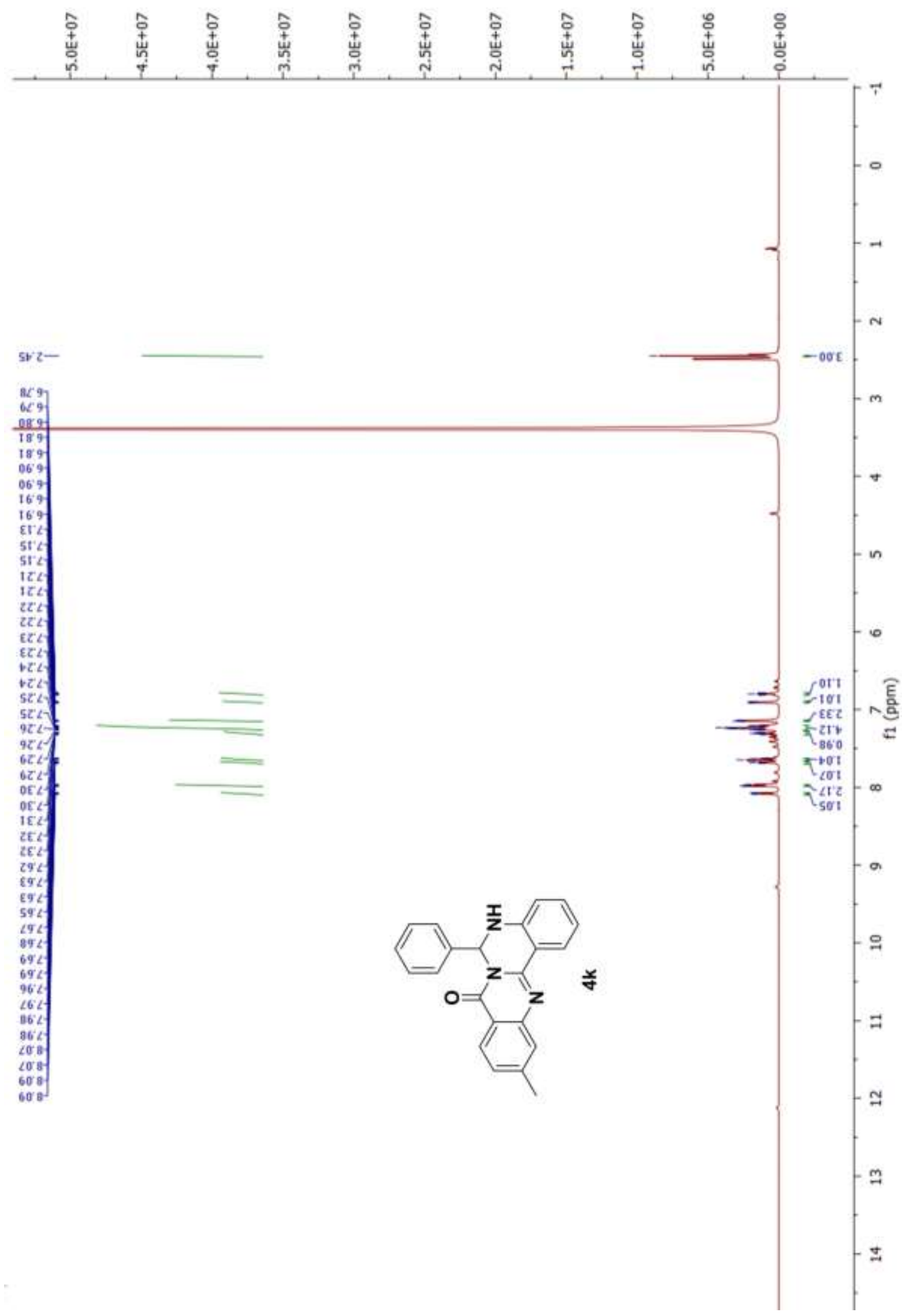


${ }^{13}$ C NMR of Compound 4k:

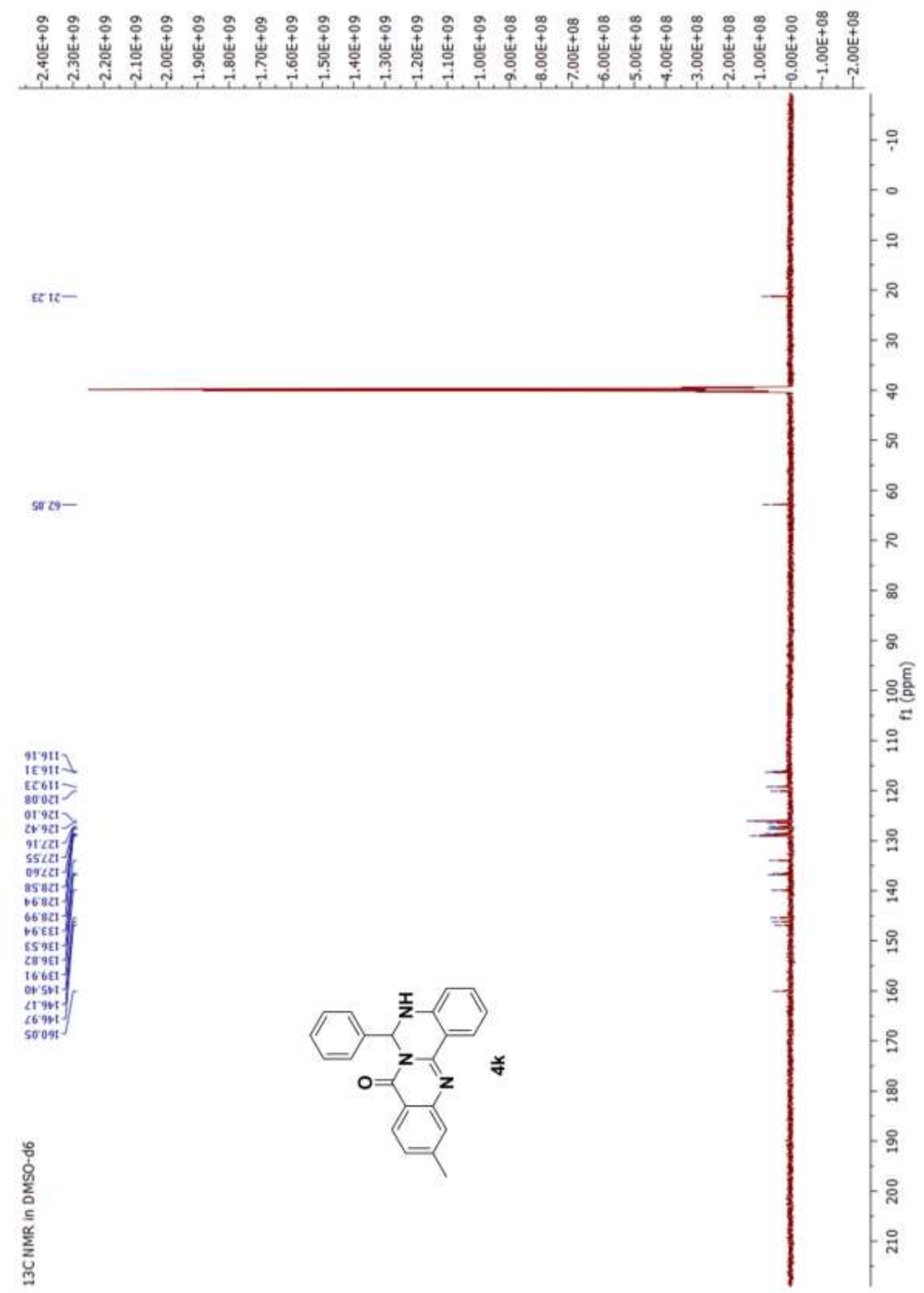


${ }^{1} \mathrm{H}$ NMR of Compound 4l:

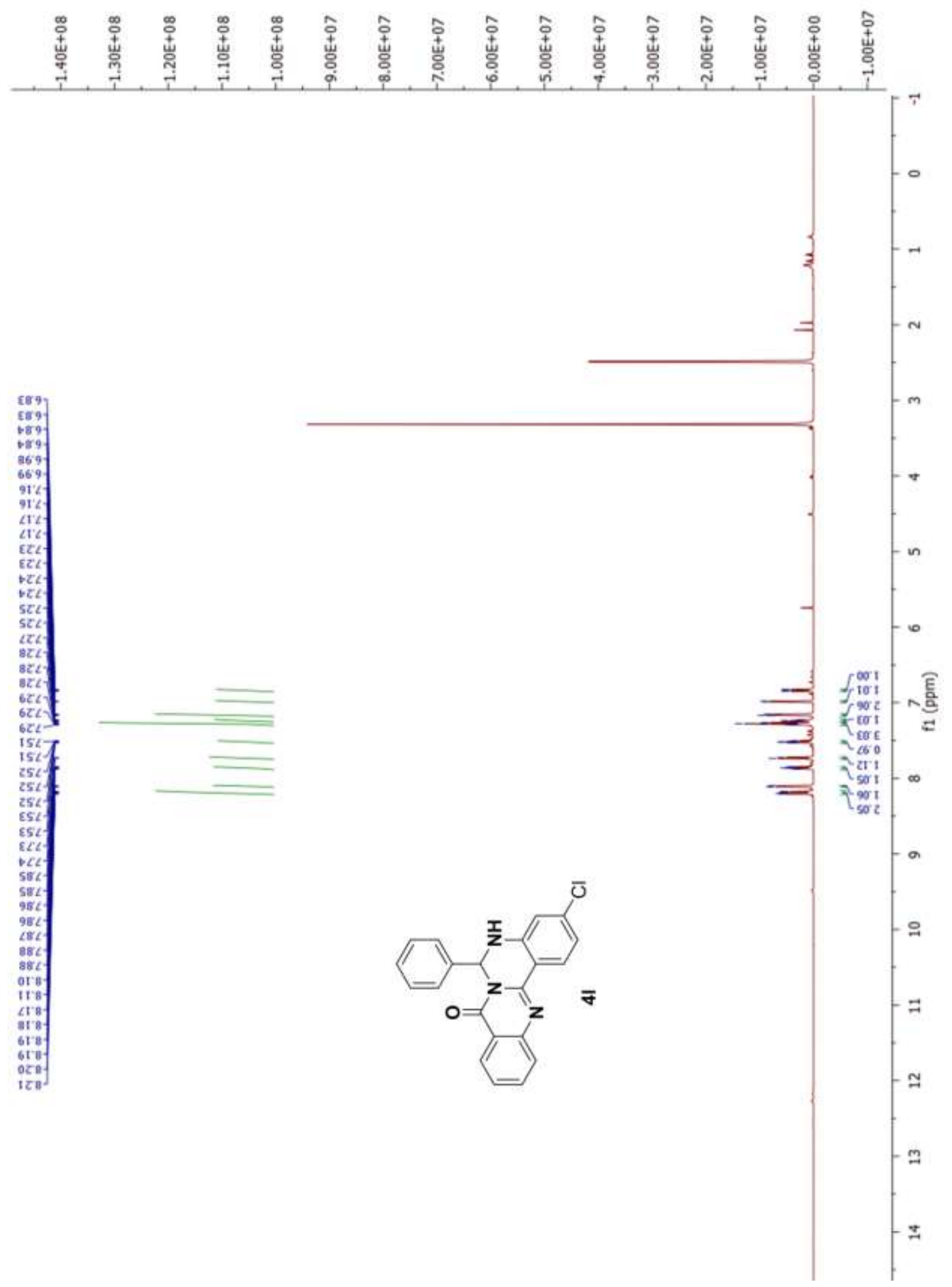


${ }^{13}$ C NMR of Compound 41:

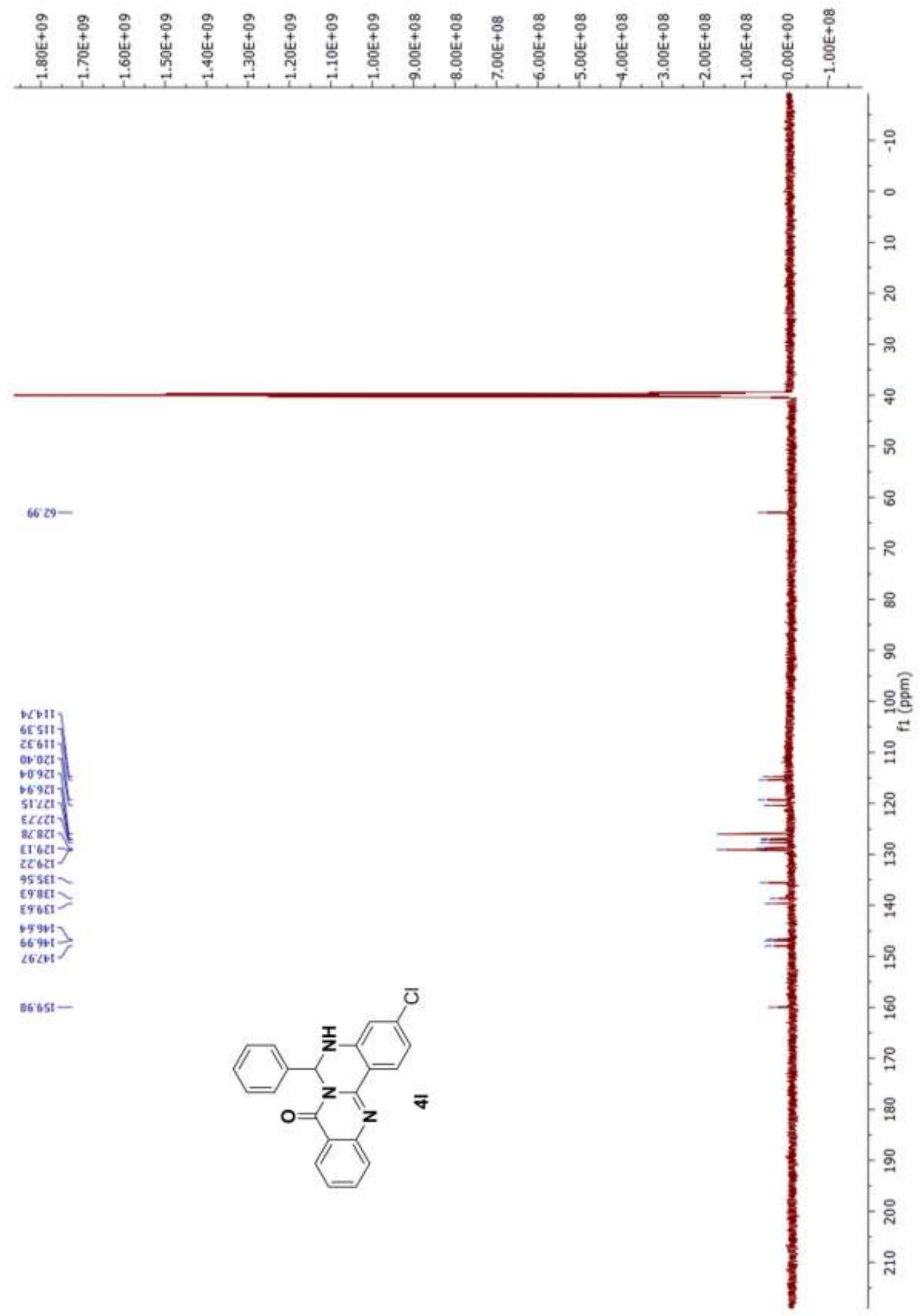


${ }^{1} \mathrm{H}$ NMR of Compound 5a:

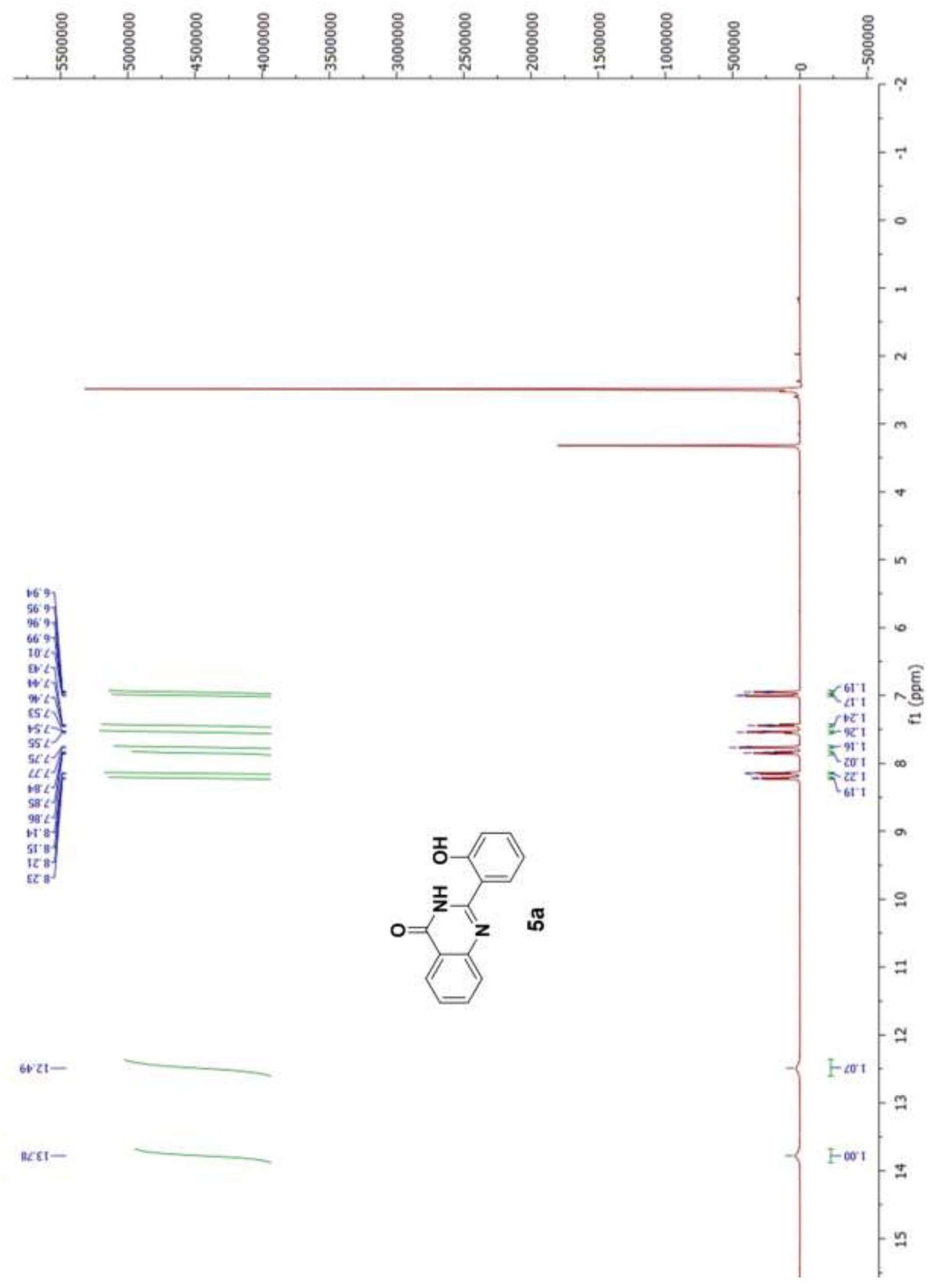


${ }^{13}$ C NMR of Compound 5a:

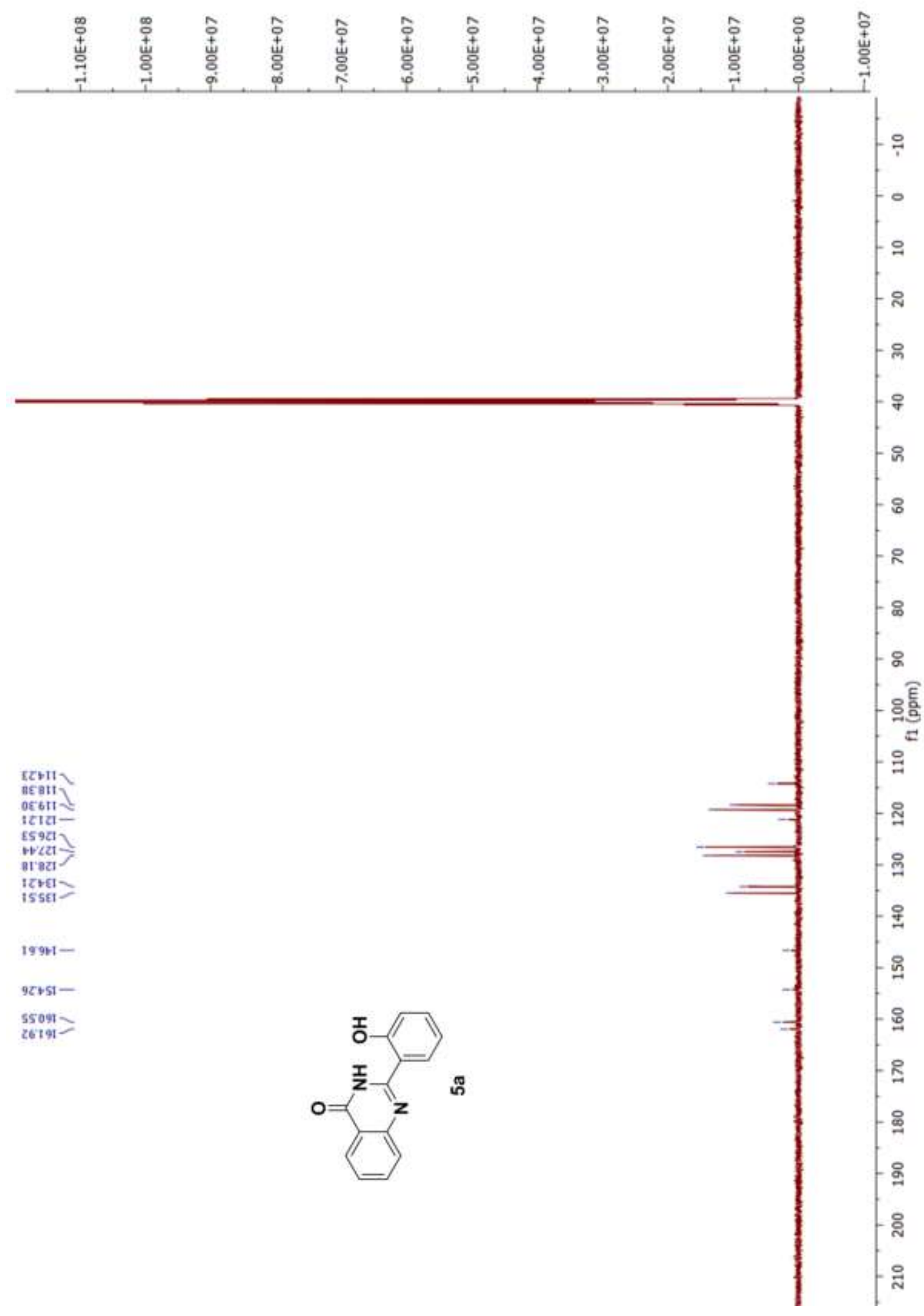


${ }^{1} \mathrm{H}$ NMR of Compound 5b:

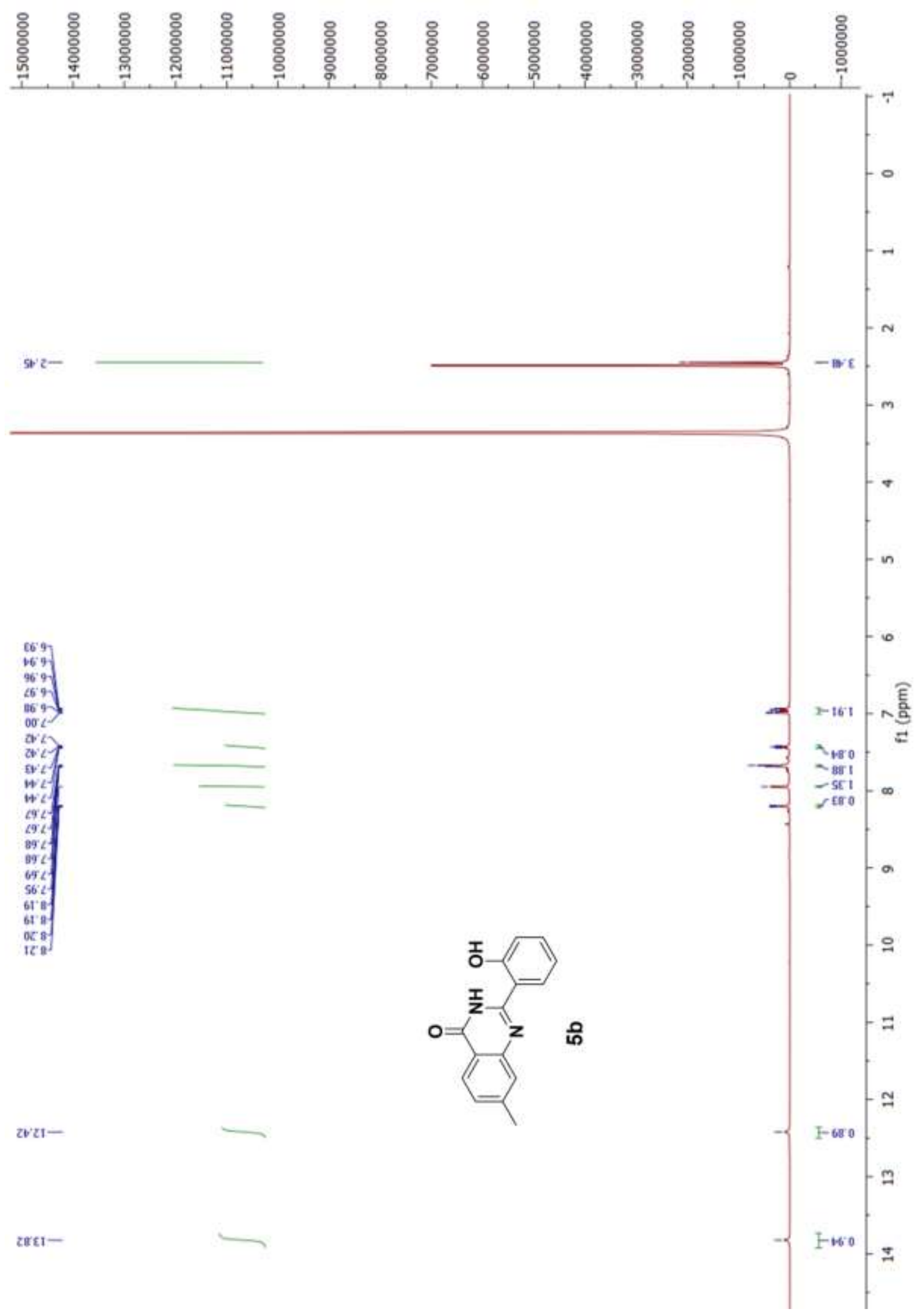


${ }^{13} \mathrm{C}$ NMR of Compound 5b:

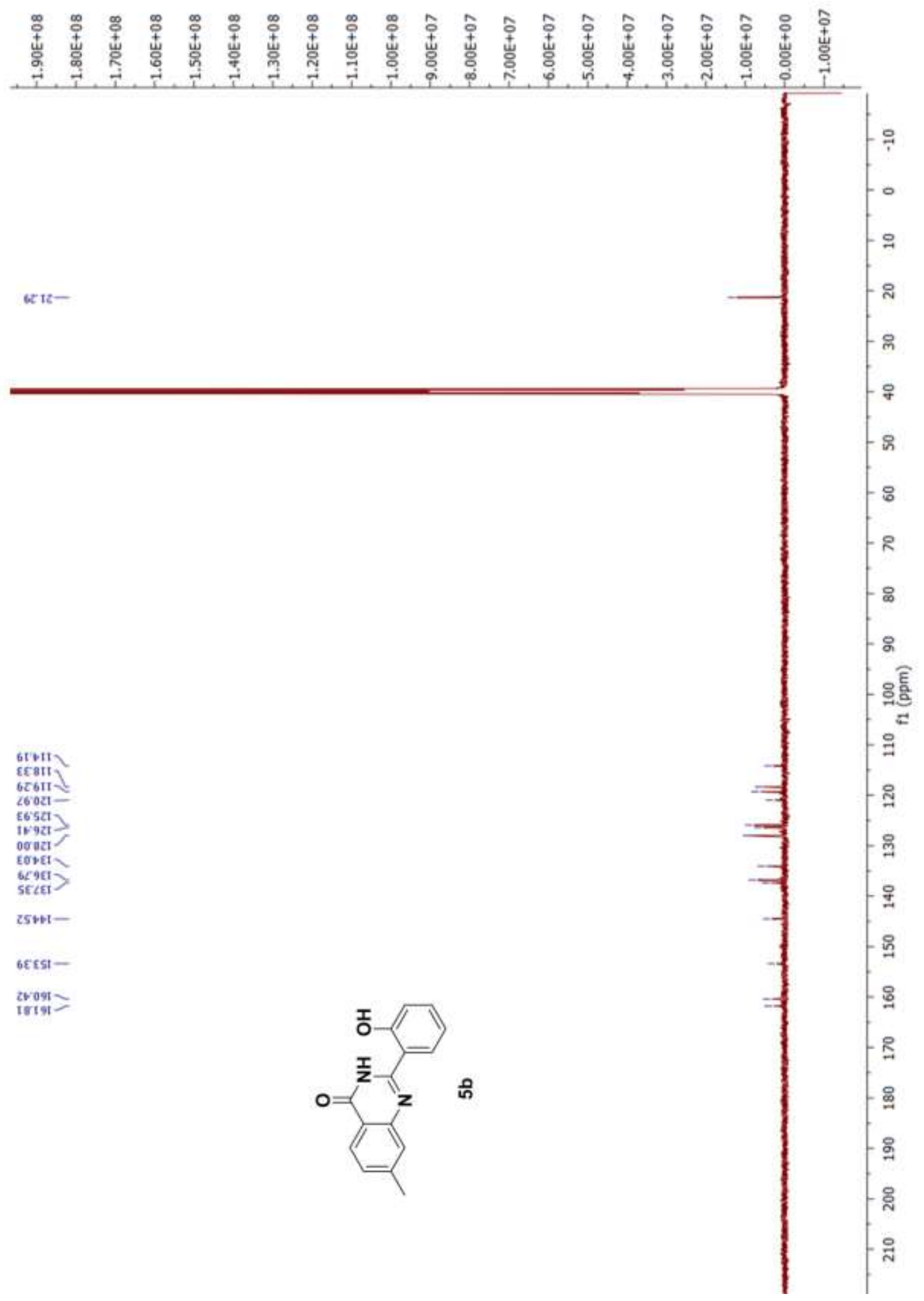


${ }^{1} \mathrm{H}$ NMR of Compound $5 \mathrm{c}$

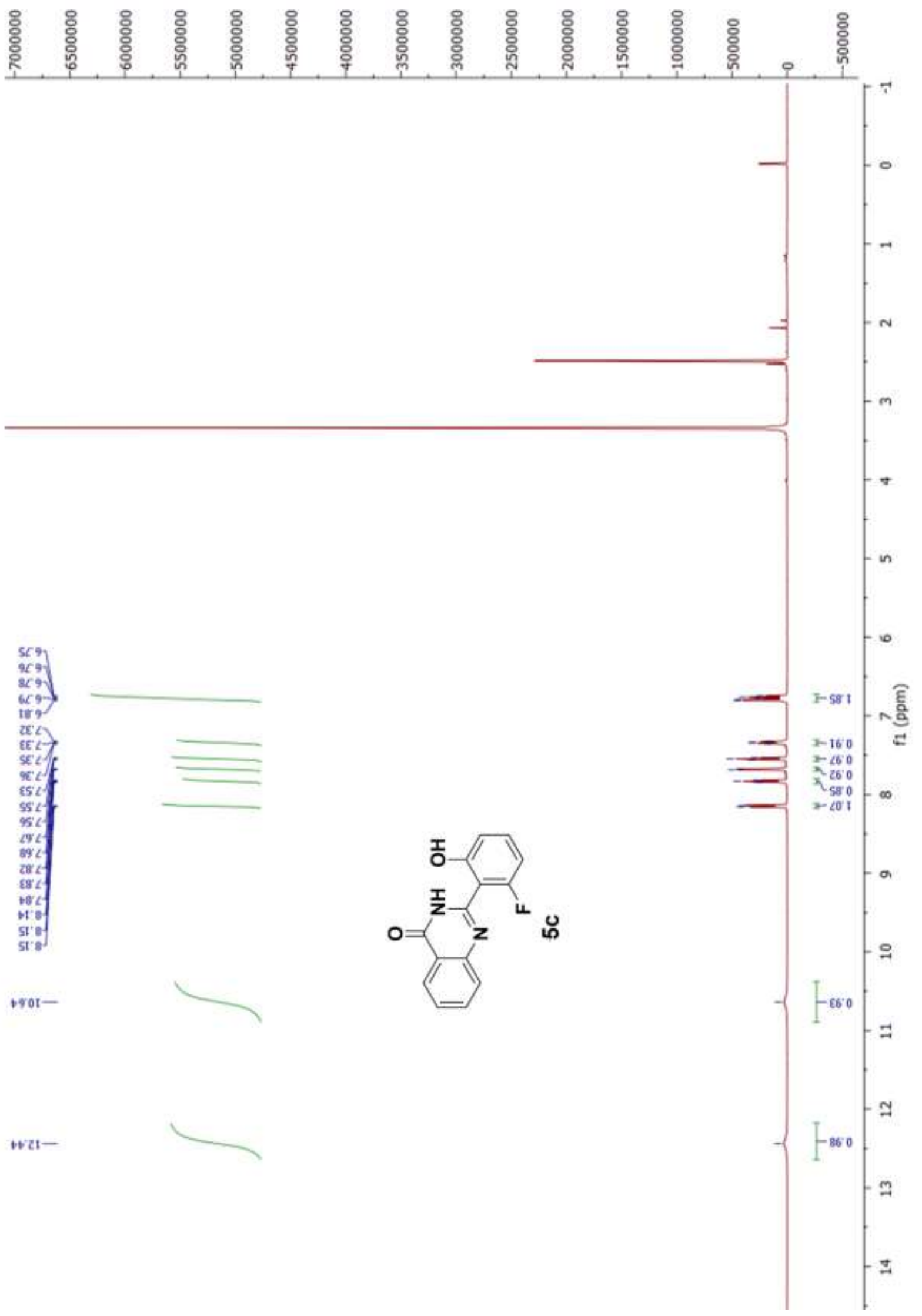


${ }^{13} \mathrm{C}$ NMR of Compound 5c:

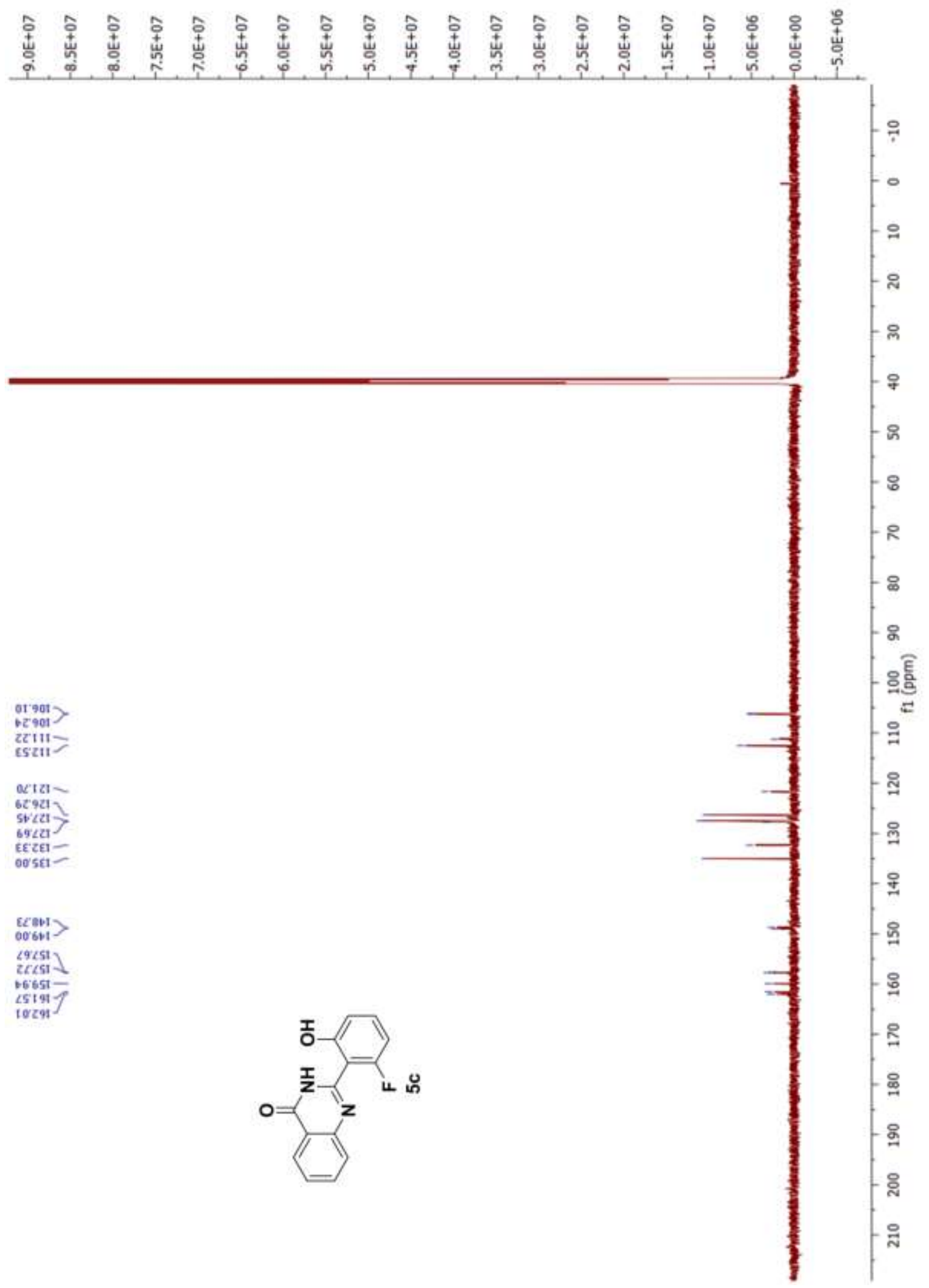

